\title{
ELEMENTARY ABELIAN $p$-SUBGROUPS OF ALGEBRAIC GROUPS
}

\author{
Dedicated to Jacques Tits for his sixtieth birthday
}

\begin{abstract}
Let $\mathbb{K}$ be an algebraically closed field and let $G$ be a finite-dimensional algebraic group over $\mathbb{K}$ which is nearly simple, i.e. the connected component of the identity $G^{0}$ is perfect, $C_{G}\left(G^{0}\right)=Z\left(G^{0}\right)$ and $G^{0} / Z\left(G^{0}\right)$ is simple. We classify maximal elementary abelian $p$-subgroups of $G$ which consist of semisimple elements, i.e. for all primes $p \neq$ char $\mathbb{K}$.

Call a group quasisimple if it is perfect and is simple modulo the center. Call a subset of an algebraic group toral if it is in a torus; otherwise nontoral. For several quasisimple algebraic groups and $p=2$, we define complexity, and give local criteria for whether an elementary abelian 2-subgroup of $G$ is toral.

For all primes, we analyze the nontoral examples, include a classification of all the maximal elementary abelian $p$-groups, many of the nonmaximal ones, discuss their normalizers and fusion (i.e. how conjugacy classes of the ambient algebraic group meet the subgroup). For some cases, we give a very detailed discussion, e.g. $p=3$ and $G$ of type $E_{6}, E_{7}$ and $E_{8}$. We explain how the presence of spin up and spin down elements influences the structure of projectively elementary abelian 2-groups in $\operatorname{Spin}(2 n, \mathbb{C})$. Examples of an elementary abelian group which is nontoral in one algebraic group but toral in a larger one are noted.

Two subsets of a maximal torus are conjugate in $G$ iff they are conjugate in the normalizer of the torus; this observation, with our discussion of the nontoral cases, gives a detailed guide to the possibilities for the embedding of an elementary abelian $p$-group in $G$. To give an application of our methods, we study extraspecial p-groups in $E_{8}(\mathbb{K})$.
\end{abstract}

\section{INTRODUCTION, NOTATION AND STATEMENT OF RESULTS}

In this article, $\mathbb{K}$ denotes an algebraically closed field and $p$ is a rational prime unequal to char( $(K)$. The symbol $i$ denotes a square root of -1 when $\operatorname{char}(\mathbb{K}) \neq 2$. By our conventions, an element of order $p$ in an algebraic group over $\mathbb{K}$ is semisimple.

We study elementary abelian $p$-subgroups of all nearly simple finitedimensional algebraic groups, classify the maximal nontoral ones and discuss aspects of their embeddings and fusion. Since our methods come from both Lie theory and finite group theory, we prefer to give a lot of detail.

It would be hard to give a historical report of interest in elementary abelian subgroups of algebraic groups. Recent references would include the 1961 paper [Bo]; many questions related to these elementary abelian subgroups for finite groups and algebraic groups in positive characteristic came up during the intense study of finite simple groups, starting in the late 1950s; see [GoLy], which lists extensive properties of finite nearly simple groups; the

Geometriae Dedicata 39: 253-305, 1991.

(C) 1991 Kluwer Academic Publishers. Printed in the Netherlands. 
1986 preprint [Ad] suggested several ideas, one of which led this author to the complexity concept (see (1.2)), which seems to be new. The classifications of finite subgroups of algebraic groups (e.g. [CoWa] and [CoGr]) raised further questions along this line. The 1974 paper [Alek] should be mentioned since it seems not to be well known; indeed the groups in the main theorem of [Alek] were discovered independently by several mathematicians (including this author).

The main results are summarized in Tables I, II and III in (1.8). The possibilities for a maximal projectively elementary abelian group follow from those tables. Throughout this article, however, are details and comments about embeddings of finite groups in algebraic groups.

We explain a bit more how to get the possibilities for a maximal nontoral elementary abelian $p$-group from Tables I and II. Let $G$ be a nearly simple algebraic group, $Z \leqslant Z(G)$ and let $E$ be a $p$-subgroup of $G$ such that $E Z / Z$ is elementary abelian and maximal such. If $E \leqslant G^{0}$, this is straightforward, though in the case $G^{0}<G$, to settle maximality of $E$, one must determine whether an outer automorphism of $G^{0}$ of order $p$ centralizes $E$. Now, assume $E$ is not in $G^{0}$. Let $F:=E \cap G^{0}$ and let $e$ generate a complement to $F$ in $E$. Then, $e$ induces an outer automorphism on $G^{0}$ and one may get the possibilities for its fixed point subgroup $H$ from Table III. One then gets $F$ by consulting Table I or II for $H$ and considering the possibility that $F$ is toral. This is straightforward, except possibly for type $D_{n}$ with $p=2$ since $H$ involves two semisimple or toral components. For types $A_{n}$ and $D_{n}$ there are many possibilities, so we do not list them in these tables. In the case of type $A_{n}$, the fixed point subgroup of $e$ has type $D_{m}$ or $C_{m}$, and we consult (2.19). In the case of type $D_{n}$ and $p=2$, we just observe that $F$ has elementary abelian image in $\mathrm{SO}(2 n, \mathbb{K})$ since it commutes with the determinant -1 action of $e$; thus, depending on $Z, F$ corresponds to a frame group or to a weakly selforthogonal binary code in the dimension $2 n$ diagonal frame group whose annihilator contains an odd diagonal transformation corresponding to the element $e$; see (2.2), (2.7) and (2.8). One does not expect to enumerate such codes explicitly. In case $G$ is disconnected of type $E_{6}$, there are two conjugacy classes of maximal nontorals not contained in $G^{0}$ since the rank 5 nontoral of a natural $F_{4}$ subgroup remains nontoral in $G^{0}$ and is conjugate to one of the two nontorals of a natural $C_{4}$ subgroup; the other nontoral becomes toral in $G$ and so is conjugate to the natural rank 7 nontoral $\mathbb{T}_{(2)}\langle t\rangle$, where $t$ inverts the ambient torus $T$. Finally, in the case of type $D_{4}$ and $p=3$, we need the result that the toral rank 2 groups in the fixed point subgroups PSL $(3, \mathbb{K})$ and $G_{2}(\mathbb{K})$ are conjugate in $G^{0}$; see (2.25).

It may happen that if we take an element $d \in E \backslash F$ such that $\langle d\rangle \neq\langle e\rangle,\langle d\rangle$ 
and $\langle e\rangle$ are not conjugate in $G$. Thus, the same group $E$ will come up in two different ways in the use of (2.18). This happens, for example, with maximal elementary abelian groups for type $D_{n}$ and $E_{6}$.

(1.1) NOTATION. When $G$ is a finite-dimensional algebraic group, $\mathbb{T}$ refers to a maximal torus of $G$ and $\mathbb{T}_{k}$ refers to a $k$-dimensional torus in $G ; \mathbb{T}$ is unique up to conjugacy, though $\mathbb{T}_{k}$ is not if $0<k<\operatorname{rk}(G)$. For an integer $n$, we let $\mathbb{T}_{(n)}$ denote $\left\{t \in \mathbb{T} \mid t^{n}=1\right\} \cong \mathbb{Z}_{n}^{\text {rk(G) }}$, and similarly define $\mathbb{T}_{k(n)}$.

(1.2) NOTATION. 'tp' refers to a tensor product situation, which includes (i) a decomposition of a vector space $V=V_{1} \otimes V_{2}$ with a bilinear form $f$ (possibly 0 ) for which each $V_{i}$ has a bilinear form $f_{i}$ (alternating or symmetric) such that $f=f_{1} \otimes f_{2}$; and (ii) some corresponding central product decomposition of groups $A=A_{1} \circ A_{2}$, where $A_{i} \leqslant \operatorname{Aut}\left(f_{i}\right)$ and $A \leqslant \operatorname{Aut}(f) \leqslant$ GL(V).

For the next definition, recall that any scalar-valued function on a finite vector space must be polynomial (as a function of the coordinates). Such a function determines a coset of the ideal of polynomial functions vanishing on the vector space; the degree is defined as the least degree of a polynomial in that coset $(-\infty$ is the degree of the 0 -function).

(1.3) NOTATION. Let $G$ be a group and let $S$ be a subset of $G$. For an elementary abelian subgroup $E$ of $G$, the complexity $\operatorname{cx}(E)$ (based on $S$ ) is the degree in the above sense of the characteristic function of $S \cap E$ in $E$. In this article, complexity is defined in a few specific situations; see Sections 6, 7, 8, 9 and 13 .

(1.4) DEFINITION. Let $A, B, \ldots$ be a (finite or infinite) sequence of conjugacy classes in the group $G$ and let $S$ be a subset of $G$. The distribution or class distribution of $S$ (with respect to the understood sequence) is the sequence of symbols $A^{a} B^{b} \ldots$, where $a=|A \cap S|, b=|B \cap S|, \ldots$ Often, $S$ is a subgroup and we restrict the sequence of classes to those which meet $S \backslash\{1\}$. In the case where $S \backslash\{1\}$ consists of elements from the conjugacy class $A$, we say that $S$ is $A$-pure. When $S$ is a group of exponent $p$ and the classes of order $p$ are designated $\mathbf{p A}, \mathbf{p B}, \ldots$ we write $A^{a} B^{b} \ldots$ for the distribution $\mathbf{p} \mathbf{A}^{a} \mathbf{p B}^{b} \ldots$

(1.5) REMARK. Orthogonality relations for finite groups are used several times; in all cases, the finite group is a p-group; its eigenvalues may be lifted to characteristic 0 , by the usual Brauer theory. In characteristic 0 , we compute the dimension of its fixed point subspace by summing traces. Actually, such a finite group may even be lifted to a subgroup of the corresponding type algebraic group in characteristic 0 (see Appendix 2). 
(1.6) DEFINITION. Assume char $\mathbb{K} \neq 2$. By $\operatorname{pin}(m, \mathbb{K})$, we mean a double cover of $\mathrm{O}(m, \mathbb{K})$ which restricts to the double cover $\operatorname{Spin}(m, \mathbb{K})$ of $\mathrm{SO}(m, \mathbb{K})$; the isomorphism type depends on whether a reflection lifts to an involution or an element of order 4 . Let $G=\operatorname{Spin}(m, \mathbb{K})$. The spin module is irreducible of dimension $2^{(m-1) / 2}$ if $m$ is odd. When $m$ is even, it decomposes into two irreducible and inequivalent modules, called half-spin modules; each has dimension $2^{m / 2-1}$. These modules are interchanged under the action of elements from $\operatorname{pin}(m, \mathbb{K}) \backslash \operatorname{Spin}(m, \mathbb{K})$. If $M$ is one of these, the kernel of the action of $G$ on $M$ is $Z$, a subgroup of $\operatorname{order} 2$ if $m \equiv 0(\bmod 4)$ and order 1 if $m \equiv 2(\bmod 4)$. The group $G / Z$ is called the half-spin group. When char $\mathbb{K}=2$, $\operatorname{pin}(n, \mathbb{K}) \cong \mathrm{O}(n, \mathbb{K})$.

(1.7) NOTATIONS. Notation for finite groups and for algebraic concepts in Lie theory are standard. The style of usage is consistent with that of [CoGr] (the relevant notations and results extend appropriately to algebraic groups) and we call attention to the following group extension notations. Let $A$ and $B$ be groups. We write $A$. $B$ for a group with normal subgroup isomorphic to $A$ and quotient $B$. In the case where the extension is split, we write $A: B$, and if nonsplit, we write $A \cdot B$. We write $n$ for a cyclic group, $p^{a}$ for an elementary abelian $p$-group, $p^{a \cdot b}$ for an elementary abelian group which is regarded as the tensor product of elementary abelian groups $p^{a}$ and $p^{b}$ (useful when the latter are a pair of modules for a pair of commuting groups), $p^{a+b+\cdots}$ for an extension of (going upward) $p^{a}$ by $p^{b}$ by $\ldots$. For a finite group $G, \exp (G)$ denotes the exponent, i.e. the least positive integer $n$ such that $x^{n}=1$, for all $x \in G$. A $p$-group $P$ is extraspecial if $P^{\prime}=Z(P)$ has order $p$; there are two isomorphism types for $P$ of a given order, which is an integer of the form $p^{1+2 r}$; we write $p^{1+2 r}$ for an extraspecial group of order $p^{1+2 r}, p_{\varepsilon}^{1+2 r}$ for such a group of type $\varepsilon= \pm$ (when $p=2$, this refers to the Witt index of the quadratic form obtained from the squaring map; when $p$ is odd, $\varepsilon=+$ iff the exponent is $p$ ). For a subset $S$ of a group, let $S^{\#}$ denote $S \backslash\{1\}$. See also [Gor], [Hup].

We let $G$ be a finite-dimensional algebraic group. If $X \in\{A, B, C, D, E, F$, $G\}, m$ and $n$ are integers such that $X_{n}$ is a type of simple algebraic group, $m X_{n}$ denotes a perfect group whose center has order $m$ and whose central quotient is the simple algebraic group of type $X_{n}$, when such a group exists. If $X, Y, \ldots$ are such symbols, $m^{a} n^{b} \ldots X_{k} Y_{l} \ldots$ denotes a perfect central extension of the semisimple group $X_{k} Y_{l} \ldots$ by a finite abelian group of type $m^{a} n^{b} \ldots$ (i.e. $\left.\mathbb{Z}_{m}^{a} \times \mathbb{Z}_{n}^{b} \times \cdots\right)$. When $p=\operatorname{char}(\mathbb{K})$ divides such $m$, it is understood that $m$ is replaced by its $p^{\prime}$ part, e.g. $Z\left(3 E_{6}(\mathbb{K})\right)=1$ when $p=3$.

We write $J_{m}+J_{n}+\cdots$ for the Jordan canonical form which is the sum of indecomposable blocks of dimensions $m, n, \ldots$ with diagonal entries 1 . We 
introduce the following departures from usual practice: $\mathrm{Dih}_{2 m}$ denotes the dihedral group of order $2 m$; Quat $_{2 m}$ denotes the quaternion group of order $2 m$.

(1.8) THE TABLES. The notational conventions are these: $G$ is a simply connected nearly simple algebraic group such that $G^{0}$ is simple modulo $Z\left(G^{0}\right)$ and $Z$ is a subgroup of $Z\left(G^{0}\right)$. We study elementary abelian $p$-subgroups of $G / Z$ by considering groups $E$ which are $p$-subgroups of $G$ such that $E Z / Z$ is elementary abelian. We discuss all maximal nontoral $E$ and a few others. We use ' $w r$ ' to indicate wreath product and $\operatorname{AGL}(n, F)$ for the affine general linear group of degree $n$ over the field $F$, i.e. the split extension of GL $(n . F)$ by the group of translations. For the definition of complexity, see (1.3) and the appropriate section $(6,7,8$ or 9). We abbreviate complexity with 'cx.'

TABLE I

Maximal nontorals for the connected case, $p=2$

\begin{tabular}{|c|c|c|}
\hline Type & $z$ & Remarks \\
\hline$A_{n}$ & $\begin{array}{l}|Z| \text { odd } \\
|Z| \text { even }\end{array}$ & $\begin{array}{l}\text { None } \\
E \text { in } 2_{\varepsilon}^{1+2 r_{\circ}} \mathbb{Z}_{2^{c}} \times 2^{s-1}, 2^{r} s=n+1, r>0, c \geqslant 1 ; \text { tp }\end{array}$ \\
\hline$B_{n}$ & $\begin{array}{l}|Z|=1 \\
|Z|=2\end{array}$ & $\begin{array}{l}E \text { corresponds to a nearly self-orthogonal even code in a } \\
\text { diagonal frame group (see }(2.7)) \\
E / Z \text { is a frame group (see }(2.7))\end{array}$ \\
\hline$C_{n}$ & $\begin{array}{l}|Z|=1 \\
|Z|=2\end{array}$ & $\begin{array}{l}\text { None } \\
E \text { has shape } 2_{\varepsilon}^{1+2 r_{\circ}} 4^{a} \times 2^{b}, 2^{r} S=2 n, r>0, a \in\{0,1\} \text {; for } \varepsilon=- \text {, } \\
(a, b)=(0, s) \text { or }(1, s / 2-1) \text {; for } \varepsilon=+, a+b=s / 2 \text {; tp; not all of } \\
\text { these are maximal; see }(2.19 . i i)\end{array}$ \\
\hline \multirow[t]{4}{*}{$D_{n}$} & \multirow{3}{*}{$\begin{array}{l}|Z|=2 \\
\text { (several } \\
\text { cases) }\end{array}$} & $\begin{array}{l}E \text { corresponds to a nearly self-orthogonal code in a diagonal } \\
\text { frame group } \\
Z \text { is the kernel of the natural } 2 n \text {-dimensional representation: } E / Z \\
\text { is a frame group for } G / Z\end{array}$ \\
\hline & & $Z$ the kernel of a half-spin representation (here, $n$ is even) \\
\hline & & $\begin{array}{l}E \text { is in one of the two natural }\left[\mathbb{T}_{1} \mathrm{SL}(n, \mathbb{K})\right] .2 \text {-subgroups } \\
\text { stabilizing a complementary pair of maximal isotropic subspaces } \\
\text { in the natural module; the image in } \mathrm{SO}(2 n, \mathbb{K}) \text { of } E \text { lies in one of } \\
\text { the subgroups Quat }{ }_{8}^{\circ} \mathrm{Sp}(n, \mathbb{K}) \text { or } \mathrm{Dih}{ }_{8}^{\circ} \mathrm{O}(n, \mathbb{K}) \text {, and has the } \\
\text { form } X \circ Y \text {, where } X \text { lies in the finite central factor and where } Y \\
\text { has shape } 2_{\varepsilon}^{1+2 r} \times 2^{s} ; \operatorname{tp} \text {; see (2.19). }\end{array}$ \\
\hline & $|Z|=4$ & $\begin{array}{l}E \text { is the preimage in } G \text { of a subgroup of } S O(2 n, \mathbb{K}) \text { of the form } \\
2_{e}^{1+2 r_{\circ}} 4^{a} \times 2^{b}, 2^{r} s=2 n, r>0 \text { and } a \in\{0,1\} ; b=s \text { if } a=0 \text {; } \\
b=s / 2-1 \text { if } a=1 ; \text { the commutator subgroup } E^{\prime} \text { may be any } \\
\text { subgroup of } Z \text {; see (2.19) and (2.22). }\end{array}$ \\
\hline$G_{2}$ & $|Z|=1$ & $\begin{array}{l}\text { For each integer } k \in\{0,1,2,3\} \text {, one conjugacy class of } \\
\text { elementary abelian subgroups of order } 2^{k} \text {; toral iff } k \leqslant 2 \text { iff } \\
\text { cx } \leqslant 2 ; \text { if } E \text { is maximal such, } N(E) \cong 2^{3} \cdot \mathrm{GL}(3,2)\end{array}$ \\
\hline
\end{tabular}


TABLE I (Cont.)

Maximal nontorals for the connected case, $p=2$

\begin{tabular}{|c|c|c|}
\hline Type & $Z$ & Remarks \\
\hline$F_{4}$ & $|Z|=1$ & $\begin{array}{l}\text { One conjugacy class of maximal elementary abelian groups, } \\
\text { represented, say, by } E \text { of rank } 5 \text {; a subgroup is toral iff the } \\
\text { complexity is at most } 2 \text { iff it does not contain a } 2 \text { A-pure eights } \\
\text { group; } N(E) \cong 2^{5} \cdot 2^{2 \cdot 3} \cdot[G L(3,2) \times G L(2,2)] \text {. }\end{array}$ \\
\hline$E_{6}$ & $|Z|=1$ or 3 & $\begin{array}{l}\text { One maximal nontoral (rank } 5 \text {, from a natural } F_{4}([\mathbb{K}) \text {-subgroup); } \\
\text { toral iff } \mathrm{cx} \leqslant 2 \text {. }\end{array}$ \\
\hline \multirow[t]{2}{*}{$E_{7}$} & $|Z|=1$ & $\begin{array}{l}\text { One maximal nontoral, rk 6, } C(E)=2^{3} \times \mathrm{SL}(2, \mathbb{K} K)^{3} \text {, } \\
N(E) / C(E)=2^{2 \cdot 3}\left[\Sigma_{3} \times \mathrm{GL}(3,2)\right] \text {. }\end{array}$ \\
\hline & $|Z|=2$ & $\begin{array}{l}\text { Two maximal nontoral (type } 1 \text { and type } 2 \text { ), both lie in a } 4 A_{7} \cdot 2 \\
\text { subgroup and in a }\left[\mathbb{T}_{1} \cdot 3 E_{6}(\mathbb{K})\right] \cdot 2 \text { subgroup; such } E / Z \text { have } \\
\text { ranks } 8 \text { and } 7 \text { and both satisfy } E^{\prime}=Z \text { and are self-centralizing; } \\
\text { we have } N(E) / E \cong 2^{7}: \mathrm{Sp}(6,2), 2^{2 \cdot 2 \cdot 1+1 \cdot 2 \cdot 3}:\left[\Sigma_{3} \times \Sigma_{3} \times \mathrm{GL}(3,2)\right] \text {, } \\
\text { respectively (in the former case, } 2^{7} \text { indicates a uniserial module } \\
\text { for } \mathrm{Sp}(6,2) \text { with ascending Löwey factors of dimensions } 6 \text { and } 1 \text { ). }\end{array}$ \\
\hline$E_{8}$ & $Z=1$ & $\begin{array}{l}\text { Two maximal elementary abelian, both nontoral, ranks } 9 \text { (type 1) } \\
\text { and } 8 \text { (type 2); an elementary abelian group is toral iff it has } \\
\text { complexity at most } 2 \text { or is not of rank } 5 \text { and } 2 \mathrm{~B} \text {-pure; both self- } \\
\text { centralizing and } N(E) / E \cong 2^{8}: \mathrm{O}^{+}(8,2) \text { if } E \text { has type } 1 \text { and } \\
N(E) / E \cong 2^{6 \cdot 2}:\left[(\mathrm{GL}(3,2) \text { wr } 2) \times \Sigma_{3}\right] \text { if } E \text { has type } 2 \text {. }\end{array}$ \\
\hline
\end{tabular}

TABLE II

Maximal nontorals for the connected case, $p$ odd

\begin{tabular}{lll}
\hline Type & $Z$ & Remarks \\
\hline$A_{n}$ & $|Z| \neq \equiv(\bmod p)$ & None \\
& $|Z| \equiv 0(\bmod p)$ & $E \cong p^{1+2 r} \circ \mathbb{Z}_{p^{2}} \times p^{s}$, where $p^{r} s=n+1$ \\
$B_{n}$ & & None \\
$C_{n}$ & & None \\
$D_{n}$ & & None \\
$G_{2}$ & & None \\
$F_{4}$ & & $3^{3} ;$ normalizer $3^{3}: \operatorname{SL}(3,3)$ \\
$E_{6}$ & $Z=1$ and $p=3$ & Two nontoral, one maximal; normalizers $3 \times 3^{3}: \operatorname{SL}(3,3)$, \\
& & $3^{1+3+3}: \operatorname{SL}(3,3) ;$ see $(11.13)$ \\
& $|Z|=3$ & Two classes $(t y p e B C$ and $t y p e A B C D)$, each of shape \\
& & $3_{+}^{1+2} \times 3^{2}, C(E) \cong 3^{3}$ and $3 \times \mathbb{T}_{2}$ and \\
& & $N(E) / E C(E)=\left[3^{2} \times 3^{2}\right]:[3 \times \mathrm{GL}(2,3)], \operatorname{Dih}{ }_{12} \times 3^{2}: \operatorname{SL}(2,3)$, \\
& & respectively; see $(11.14)$.
\end{tabular}


TABLE II (Cont.)

Maximal nontorals for the connected case, $p$ odd

\begin{tabular}{|c|c|c|}
\hline Type & $Z$ & Remarks \\
\hline$E_{7}$ & $|Z|=1$ or $2 ; p=3$ & $\begin{array}{l}\text { Two classes of nontoral } E \text {, one contained in the other: } \\
\text { rk } E=3 \text { or } 4 \text { with the same centralizer } 3^{3} \times \mathbb{T}_{1} \\
N(E)=\left[3^{3}: \operatorname{SL}(3,3) \times \mathbb{T}_{1}\right]: 2 \text { if } \mathrm{rk} E=3 \text { (this maps onto } \\
\left.3^{3}: \mathrm{GL}(3,3) \text { and } \mathrm{O}(2, \mathbb{K})\right) \\
N(E)=\left[3^{1+3+3}: \operatorname{SL}(3,3) \circ \mathbb{T}_{1}\right]: 2 \text { if } \mathrm{rk} E=4\end{array}$ \\
\hline$E_{8}$ & $p=3$ & $\begin{array}{l}\text { Two maximal nontoral, both of rank } 5 \\
\text { type 1: centralizer } 3^{1+3+3} \circ \mathbb{T}_{2} ; \\
\quad \text { normalizer }\left[3^{1+3+3}: \operatorname{SL}(3,3) \circ \mathbb{I}_{2}: 3\right]: 2 \\
\text { (amalgamations over } \mathbb{Z}_{3} ; N(E) \text { has AGL }(3,3) \text { and } \Sigma_{3} \text { as } \\
\text { quotients) } \\
\text { type 2: } C(E)=E, N(E) / E=3^{4}:[\operatorname{Sp}(4,3) \cdot 2] \text { (has } W_{E_{6}} \text { as } \\
\text { quotient) }\end{array}$ \\
\hline & $p=5$ & One nontoral, order $5^{3} ; C(E)=E$ and $N(E)=5^{3}: \operatorname{SL}(3,5)$ \\
\hline
\end{tabular}

TABLE III

Maximal nontorals for the disconnected case

( $G$ almost simple, $E$ projectively elementary abelian, $F=E \cap G^{0}, E=F \times\langle e\rangle,|e|=p$; we use (2.18) to give fixed points of $e$ on $G^{0}$, then for the possibilities for nontoral $F$, refer to (2.19) for type $A$, (5.4.ii) for type $D$ and Section 8 and (2.19) for type $E_{6},(2.25)$ for $p=3$ and $D_{4}$ )

\begin{tabular}{|c|c|c|}
\hline Type & & Remarks \\
\hline$A_{n}$ & $p=2 ;$ many $E$ & $\begin{array}{l}\text { For }|Z| \text { odd, fixed points } \mathrm{Sp}(n+1, \mathbb{K})(n \text { odd }) \text { or } \mathrm{SO}(n+1, \mathbb{K}) \text {; for } \\
|Z| \text { even, }(n+1) /|Z| \text { odd, fixed points } \mathrm{PSp}(n+1, \mathbb{K}) \text { or } \\
\mathrm{PO}(n+1, \mathbb{K}) ; \text { for }|Z| \text { even, }(n+1) /|Z| \text { even, fixed points } \\
\operatorname{PSp}(n+1, \mathbb{K}) \times 2 \text { or } \operatorname{PSO}(n+1, \mathbb{K}) \times 2 \text {. }\end{array}$ \\
\hline$D_{n}$ & $p=2 ;$ many $E$ & $\begin{array}{l}\text { for } Z=1 \text {, fixed points } \\
{[\operatorname{Spin}(k, \mathbb{K}) \circ \operatorname{Spin}(2 n-k, \mathbb{K})] \text { for an odd integer } k, k \leqslant n / 2 \text {; for }} \\
|Z|=2, \text { fixed points } \\
2 \times \operatorname{SO}(k, \mathbb{K}) \times \operatorname{SO}(2 n-k, \mathbb{K}) \text { for an odd integer } k, k \leqslant n / 2 \text {; for } \\
|Z|=4, \text { fixed points } \\
\operatorname{SO}(k, \mathbb{K}) \times S O(2 n-k, \mathbb{K}) \text { for an odd integer } k, k \leqslant n / 2 \text {. } \\
\text { (two classes of groups } E \text { ) } \\
\text { Two classes of outer elements of order } 3 \text {, with fixed points } G_{2}(\mathbb{K}) \\
\text { (one such } F \text { ) and PSL }(3, \mathbb{K}) \text { (two such } F \text { ). }\end{array}$ \\
\hline$E_{6}$ & $p=2$ & $\begin{array}{l}\text { (two classes of such } E \text {; ranks } 6 \text { and } 7 \text { ) } \\
\text { Two classes of outer involutions, with fixed points } F_{4}(\mathbb{K}) \text { (one } \\
\left.\text { such maximal } F \text {, nontoral in } G^{0}\right) \text { and } \operatorname{PSp}(8, \mathbb{K})(\text { two such } \\
\text { maximal } F \text {, both nontoral in } \operatorname{PSp}(8, \mathbb{K}) \text {; one becomes } \mathbb{T}_{(2)} \text { in } G^{0} \text {, } \\
\text { the other lies in an } F_{4}(\mathbb{K}) \text { fixed point subgroup) }\end{array}$ \\
\hline
\end{tabular}


TABLE IV

Maximal extraspecial subgroups of $E_{8}(\mathbb{K})$

\begin{tabular}{lll}
\hline Prime & Group $p_{\varepsilon}^{1+2 d}$ & Remarks \\
\hline$p \geqslant 11$ & & None (see Section 12) \\
$p=7$ & $d=1, \varepsilon= \pm$ & Several \\
$p=5$ & $d=1, \varepsilon= \pm$ and $d=2, \varepsilon= \pm$ & Several \\
$p=3$ & $d=1$ or $2, \varepsilon= \pm$ & Several \\
$p=2$ & & Two maximal with center of type $2 \mathrm{~B}:(d, \varepsilon)=(3,+)$ \\
& & and (7, +$)$ \\
& & several with center of type $2 \mathrm{~A}: d \leqslant 2$ \\
\end{tabular}

(1.9) NOTATION AND TERMINOLOGY FOR THE REMAINDER OF THE PAPER

We let $G$ be a nearly simple finite-dimensional algebraic group over the algebraically closed field $\mathbb{K}$ such that $G^{0}$ is simply connected; $G$ refers to the main group under consideration in each section of this article. The prime $p$ is always different from char( $\mathbb{K})$.

$H$ usually means an algebraic group containing $G$.

$Z$ is a subgroup of $Z\left(G^{0}\right)$.

$E$ is a projectively elementary abelian $p$-subgroup of $G$, i.e. a $p$-group whose image in $G / Z$ is elementary abelian.

For a subset $S$ of $G \leqslant H, C(S)$ and $N(S)$ refer to centralizer and normalizer in $G$; otherwise, we use subscripts: $C_{H}(S)$, etc.

A fundamental $\operatorname{SL}(2, \mathbb{K})$ means a conjugate in $G$ of an $\operatorname{SL}(2, \mathbb{K})$ which is generated by a pair of root groups.

(1.10) ACKNOWLEDGEMENTS. It is a pleasure to thank Arjeh Cohen for many interesting and informative conversations during our collaboration on finite subgroups of Lie groups; Gary Seitz for the preprint of [CoLiSaSe]; Ferdinand Veldkamp, Tonny Springer and the referee for helpful suggestions; participants in my seminars at Ann Arbor and other places; the University of Michigan and the National Science Foundation for financial support; the Centre Nationale du Recherche Scientifique of France for support during my visit at the École Normale Supérieure, 1986-87, when this work was initiated.

\section{Preliminary results}

(2.1) DEFINITIONS. For basic definitions from coding theory, see [MacWS1]. We mention the standard term doubly even code for a binary code (i.e. over $\mathbb{F}_{2}$ ) whose every element has weight divisible by 4 ; such codes are not 
classified explicitly in general, even the self-orthogonal ones. We also use the term nearly self-orthogonal for a code $C$ such that $\operatorname{dim}\left(C^{\perp} / C\right) \leqslant 1$.

For later calculations, we need to classify (up to equivalence) certain smalldimensional binary codes and get their groups. We leave as an exercise the claim that the list of codes below has the stated properties and exhaust all nearly self-orthogonal codes of the given lengths.

(2.2) TABLE

TABLE V

Small-dimensional nearly self-orthogonal binary codes

\begin{tabular}{llll}
\hline Length & Dimension & Basis & Group \\
\hline 2 & 1 & 12 & $\Sigma_{2}$ \\
3 & 1 & 12 & $\Sigma_{2}$ \\
4 & 2 & 12,34 & 2 wr 2 \\
5 & 2 & 12,34 & 2 wr 2 \\
6 & 3 & $12,34,56$ & 2 wr $\Sigma_{3}$ \\
7 & 3 & $12,34,56$ & $2 \mathrm{wr} \Sigma_{3}$ \\
& 3 & $1234,1256,1357$ & $\mathrm{GL}(3,2)$ \\
8 & 4 & $12,34,56,78$ & $2 \mathrm{wr} \Sigma_{4}$ \\
& 4 & $1234,1256,1357,12345678$ & $\operatorname{AGL}(3,2) \cong 2^{3}: \mathrm{GL}(3,2)$ \\
9 & 4 & $12,34,56,78$ & $2 \mathrm{wr} \Sigma_{4}$ \\
& 4 & $1234,1256,1357,89$ & $\mathrm{GL}(3,2) \times \Sigma_{2}$ \\
$10(=X)$ & 4 & $1234,1256,1357,12345678$ & $\operatorname{AGL}(3,2)$ \\
& 5 & $12,34,56,78,9 \mathrm{X}$ & $2 \mathrm{wr} \Sigma_{5}$ \\
& 5 & $1234,5678,1357,12345678,9 \mathrm{X}$ & $\operatorname{AGL}(3,2) \times 2$ \\
\hline
\end{tabular}

(2.3) DEFINITIONS. (Famous codes.) The Hamming code (with parameters $[7,3,4]$ ) is the unique self-orthogonal binary code with parameters $[7,3,4]$. Its group is $\operatorname{GL}(3,2)$ and the code may be thought of as the set of complements to linear subspaces of codimension 1 in $\mathbb{F}_{2}^{3}$, together with the empty set, with Boolean sum as the addition. The extended Hamming code is the unique self-orthogonal $[8,4,4]$-binary code; it is spanned by a copy of the Hamming code supported on seven of the alphabet letters and $(1,1,1,1,1,1,1,1)$. Its group is $\operatorname{AGL}(3,2) \cong 2^{3}: \mathrm{GL}(3,2)$.

The tetracode is the unique self-orthogonal length 4 ternary code; equivalently, it is the unique $[4,2,3]$ ternary code. Any nonzero vector has weight 3 and the standard version of this code is spanned by $(s, a, a+s, a+2 s)$, for $a$, $s \in \mathbb{F}_{3}$. Its group is $\mathrm{GL}(2,3)$,

(2.4) LEMMA. (i) Let $T$ be a finite p-group whose Frattini subgroup is cyclic and central. Then $T^{\prime}$ has order 1 or $p$ and there are subgroups $X, Y$ such that 
$T=X \circ Y$, where $X$ is extraspecial, $Y$ has an abelian maximal subgroup and $\Omega_{1}(Y)$ is elementary abelian.

(ii) If $T / T^{\prime}$ is elementary abelian, $Y$ is of the form $p^{r}$ or $\mathbb{Z}_{p^{2}} \times p^{r}$.

Proof. (i) Notice that $T^{\prime}$ has order 1 or $p$. The abelian case is trivial, so we assume that $T^{\prime}$ has order $p$. Let $U \geqslant T^{\prime}$ satisfy $U / T^{\prime}=\Omega_{1}\left(T / T^{\prime}\right)$. Let $E$ be an extraspecial subgroup of $U$ such that $U=Z(U) E$. Then, $[E, T]=T^{\prime}=Z(E)$ implies that $T=C(E) E$. Since $T / U$ is cyclic and $T^{\prime}$ has order $p$, we see that $|C(E): C(E) \cap C(Z(U))|=1$ or $p$. Since $\Phi(T)$ is cyclic, the same is true for subgroups and quotients, whence $C(E) \cap C(Z(U))$ is central-by-cyclic, hence abelian. Take $X=E$ and $Y=C(E)$.

(ii) follows easily since $Y=Z(U)$ if $T / T^{\prime}$ is elementary abelian.

(2.5) DEFINITION. Let $G=G_{1} \circ \cdots \circ G_{n}$ be a central product and define $G^{i}:=\left\langle G_{j} \mid j \neq i\right\rangle, Z_{i}:=G_{i} \cap G^{i}$ and $Z:=\left\langle Z_{i} \mid i=1, \ldots, n\right\rangle \leqslant Z(G)$. There is a natural map $G \rightarrow \Pi G_{i} / Z_{i}$, defined by $\left(g_{i}\right) \mapsto\left(Z_{i} g_{i}\right)$ (easily, one sees this is well defined) with kernel $Z$. Let $S$ be a subgroup of $G$. The quasiprojections of $S$ are the groups $S_{i} \leqslant G, i=1, \ldots, n$, which satisfy: $Z_{i} \leqslant S_{i}$ and $S_{i} / Z_{i}$ is the projection of $S Z / Z$ into the $i$ th factor with respect to the decomposition $\Pi G_{i} / Z_{i}$.

(2.6) LEMMA. Let $\Omega$ be an m-set and let $P \Omega, P E \Omega$ be the vector space of subsets, even subsets, respectively, with addition the Boolean sum and with the natural bilinear form $(A, B) \mapsto|A \cap B|(\bmod 2)$ on $P \Omega$ and quadratic form on $P E \Omega(A \mapsto 1 / 2|A|(\bmod 2))$.

(i) If $m$ is odd, the bilinear form on PES is nonsingular and the quadratic form has maximal Witt index (plus type) iff $m \equiv \pm 1(\bmod 8)$.

(ii) If $m$ is even, there is a well-defined quadratic form induced on $P E \Omega /\langle\Omega\rangle$ iff $m \equiv 0(\bmod 4) ;$ it has maximal Witt index iff $m \equiv 0(\bmod 8)$.

Proof. This is well known. See [Gr2], for instance.

(2.7) DEFINITION. Let $\operatorname{char}(\mathbb{K}) \neq 2$ and let $V$ be an $m$-dimensional vector space with nondegenerate bilinear form $f$ and orthonormal basis $\mathscr{B}=\left\{e_{i} \mid i \in \Omega\right\}$, where $\Omega=\{1, \ldots, m\}$. We call $\mathscr{B}$ a frame. A signed frame is a set of the form $\pm \mathscr{B}$. The associated frame group is the subgroup of Aut $(f)$ stabilizing $\pm \mathscr{B}$; it is isomorphic to $2 \mathrm{wr} \Sigma_{n}$. The frame group is a monomial group which is a semidirect product of the diagonal frame group (diagonal as matrices with respect to $\mathscr{B}$ ) and the natural group of permutation matrices. (See (5.1).) The elements $\varepsilon_{i}$ of $D$ which satisfy $e_{i} \varepsilon_{j}=e_{i}$ or $-e_{i}$ as $j \neq i, j=i$, form a basis for $D$ and give $D$ the structure of $\mathbb{F}_{2}^{n}$; a subgroup of $D$ is naturally identified with a code, so the code-theoretic notions of weight, etc., apply to $D$. 
(2.8) REMARK. The preimage in $\operatorname{pin}(m, \mathbb{K})$ of $D$ from (2.7) is, roughly speaking, extraspecial and may be analyzed by these principles: (a) if $r$ and $s$ are lifts of distinct commuting reflections, $[r, s]$ generates the kernel of the action on the natural module; (b) if $u$ is the lift of an involution in $\operatorname{SO}(m, \mathbb{K})$ with multiplicity $k$ for the eigenvalue -1 , then $|u|=2$ or 4 as $k \equiv 0$ or 2 $(\bmod 4)$. For background, see [Sch1,2,3], [Chev], [St]; also see [Wood]; [GarGag] studies the interesting question of getting a nonsplit double cover of a finite group by taking its orthogonal representations.

(2.9) LEMMA. Let char $\mathbb{K} \neq 2$ and let $G$ be $\operatorname{Spin}(m, \mathbb{K}), n=\operatorname{rank} G$ and let $V$ be the natural m-dimensional module. Suppose $f \in G$ and $f^{2}$ is -1 on $V$. Then $m=2 n$ is even. If $C:=C_{G}(f Z(G) / Z(G)), C \cong \mathbb{T}_{1} \mathrm{SL}(n, \mathbb{K})$ for $n$ odd and $C \cong \mathbb{T}_{1} \mathrm{SL}(n, \mathbb{K}): 2$ for $n$ even. Also, $[C, f]$ is a subgroup of $Z(G) ;[C, f]$ is trivial if $n$ is odd and when $n$ is even, $[C, f]=\left\langle f^{2}\right\rangle$ is cyclic of order 2 and is the kernel of a half-spin representation. There are two conjugacy classes of such $f$ and they are interchanged by outer automorphisms.

Proof. On $V, f$ has eigenvalues $i$ and $-i$ only. Since an eigenspace is nonsingularly paired with its dual, $m$ must be even. Let $Z$ be the kernel of the action of $G$ on $V ;|Z|=2$.

Let $D:=C_{G}(f) ; D$ is connected by (2.13.v). It is normal in $C$, has index at most 2 (since the image of $f$ in $\operatorname{SO}(2 n, \mathbb{K})$ has centralizer isomorphic to $\mathrm{GL}(n, \mathbb{K} K))$ and $C / D$ is identified with a subgroup of $\operatorname{Hom}\left(\langle f\rangle\left\langle\left\langle f^{2}\right\rangle, Z\right)\right.$.

If $n$ is odd, $\left\langle f^{2}\right\rangle=Z(G) \cong \mathbb{Z}_{4}$ and $f \in Z(D) \cong \mathbb{T}_{1}$. Suppose $g \in C \backslash D$. Thus, $f^{g}=f^{k}$, for an integer $k \not \equiv 1(\bmod |f|)$; since $g^{2} \in D, k^{2} \equiv 1(\bmod |f|)$. We may choose $g$ to satisfy $g^{2} \in\left\langle f^{4}\right\rangle$. Since $g$ acts on $Z(D) \cong \mathbb{T}_{1}, k= \pm 1$ and so $k=-1$. Since $g^{2} \in Z, g$ induces an involution on $V$ of determinant $(-1)^{n}=-1$, a contradiction.

Suppose $n$ is even. Then $D \cong \mathbb{T}_{1} \times \operatorname{SL}(n, \mathbb{K}), Z(D) \cong \mathbb{T}_{1} \times n$ and $|f|=4$; also we may assume that $f \in Z(D)^{0}$, whence $|C / D| \leqslant 2$. There is an element $g \in G$ such that $\langle g, f\rangle Z / Z \cong \operatorname{Dih}_{8}$. Therefore, $|C / D|=2$ and so $[C, f]$ has order 2, as claimed. To prove that $[C, f]$ is the kernel of a half-spin representation, we observe that it has order 2 and maps to $\left\langle-1_{V}\right\rangle$ in the natural representation, hence is not the kernel of the action on the natural module. The last statement follows from the well-known fact that the orbit of $O(2 n, \mathbb{K})$ on maximal isotropic subspaces splits into two orbits for $\mathrm{SO}(2 n, \mathbb{K})$.

(2.10) DEFINITION. Let char $\mathbb{K} \neq 2$. We call an element $g \in G=\operatorname{Spin}(m, \mathbb{K})$ imaginary if $g^{2}$ acts as -1 on the natural $m$-dimensional module. Such elements exist if $m=2 n$ is even and they have orders 8 or 4 as $n$ is odd, even, respectively. Let $n$ be even. We say two imaginary elements $f$ and $g$ have the same spin if $f^{2}=g^{2}$; otherwise, opposite spins. We call the two different spins 
$u p$ and down, terms suggested by the upper and lower branch at the forked end of the $D_{n}$ Dynkin diagram. Having the same spin is the same as being conjugate; see (2.9).

(2.11) LEMMA. (Quotients of Bilinear Forms.) Let $G=H \circ K$ be a central product of groups with finite dimensional $\mathbb{K} G$-module $V$. Assume that every finite-dimensional module for $H$ or $K$ is completely reducible. Suppose that $\left.V\right|_{H}$ is a direct sum of modules, each isomorphic to the module $M$ such that $\operatorname{dim} \operatorname{Hom}_{H}(M \otimes M, \mathbb{K})=1$. Let $h$ be a nonzero $H$-invariant bilinear form on $M$ and assume that $V$ has a $G$-invariant bilinear form $g$. Then, there is a module $N$ for $K$ with an invariant bilinear form $k$ such that $V \cong M \otimes N$ and $g=h \otimes k$.

Proof. There is a module $N$ for $K$ such that $V \cong M \otimes N$, with the natural action of the central product (decompose $V$ into a direct sum of $H$ homogeneous components to see this). Since $\operatorname{dim} \operatorname{Hom}_{H}(M \otimes M, \mathbb{K})=1$ and the fixed point subspace for $G$ on $V \otimes V$ is naturally isomorphic to $(M \otimes M)^{H} \otimes(N \otimes N)^{K}$, the result follows.

(2.12) LEMMA. Notation as in (2.9) with $m=2 n \equiv 0(\bmod 4)$. Let $f$ and $g \in C$ be imaginary. If $g \notin D, f$ and $g$ have the same spin. If $g \in D, f g^{-1}$ acts on the natural module Vas an involution, say, with eigenvalues $\left(-1^{2 a}, 1^{2 n-2 a}\right)$; then, $f$ and $g$ have the same spin iff $a$ is even.

Proof. Suppose $g \notin D$. Then $\langle f, g\rangle \cong \mathrm{Quat}_{8}$ and $C(\langle f, g\rangle) \cong \mathrm{Sp}(n, \mathbb{K})$. We may decompose $V$ as $V_{1} \otimes V_{2}$ where the factors are modules for $C(\langle f, g\rangle)$ and $C(C(\langle f, g\rangle) \cong \mathrm{Sp}(2, \mathbb{K}) \cong \mathrm{SL}(2, \mathbb{K})$, respectively. In the latter group, the normalizer of $\langle f, g\rangle$ is isomorphic to $\operatorname{SL}(2,3)$, whence $f$ and $g$ are conjugate.

Now assume that $f$ and $g$ commute. For symbols $j, k= \pm$, let $V_{j k}$ denote the subspace of $V$ where $f$ acts as $i^{j 1}$ and $g$ acts as $i^{k 1}$. There is $x \in \operatorname{pin}(m, \mathbb{K})$ such that $f^{x}=g$. We may assume that $x$ is trivial on $V_{++}$since the stabilizer in $G$ of an eigenspace $W$ of $f$ or $g$ acts as $\mathrm{GL}(W)$ there; also, $x$ acts trivially on $V_{--}$ since $V_{--}$is in perfect duality with $V_{++}$. Then, $x$ stabilizes $V_{++}^{\perp_{+}}=V_{++} \oplus V_{+-} \oplus V_{-+}$and sends the second factor to the third factor. If $\operatorname{dim}\left(V_{++}\right)>0$, induction applies to the proper subspace $V_{+-} \oplus V_{-+}$. Assume that $\operatorname{dim}\left(V_{++}\right)=0$. Then, $f^{-1}$ acts as -1 on $V$ and $a=n$. By decomposing $V$ as a direct sum of $\langle f, g\rangle$-invariant hyperbolic planes, we see that $x r$ centralizes $f$, where $r$ is a product of $n$ reflections interchanging eigenspaces for $f$ on those $n$ planes. The result follows since the stabilizer of an isotropic subspace in $\mathrm{GO}(2 n, \mathbb{K})$ lies in $\mathrm{SO}(2 n, \mathbb{K})$, due to the fact that the graph automorphism interchanges the two conjugacy classes of parabolics which stabilize maximal isotropic subspaces.

Now we recall some basic results from the theory of Lie groups and algebraic groups. 
(2.13) PROPOSITION. Let $G$ be a finite-dimensional algebraic group and let $H$ be a finite-dimensional connected algebraic group.

(i) Let $G$ be reductive. If $A$ is an abelian subgroup and $x$ is a semisimple element of $A, A$ is toral in $G$ iff $A$ is toral in $C(x)$. If $A$ is toral, $N(A) / C(A)$ is embedded in $N(T) / C(T)$, for a torus $T$ of $C(A)$.

(ii) Let $H$ be reductive. Every semisimple element lies in a torus.

(iii) If $S$ is a finite supersolvable group of semisimple elements acting on $G, S$ normalizes a maximal torus. (Recall that finite nilpotent groups are supersolvable.)

(iv) Suppose that $H$ is a simply connected semisimple algebraic group with root lattice $L$ and that $K$ is a semisimple subgroup generated by root groups, with root lattice $M \leqslant L$. If $M$ is a direct summand of $L$ as abelian groups, $K$ is simply connected.

(v) If $H$ is simply connected and reductive and $x$ is a semisimple element, $C(x)$ is connected (but not necessarily simply connected). If $|x|$ is finite of order relatively prime to the order of the finite group $Z\left(H^{\prime}\right)$, then $C_{H / Z}(x Z)$ is connected, for any closed $Z \leqslant Z(H)$.

(vi) If $H$ is semisimple and $A$ is a finite abelian subgroup of semisimple elements with at most two generators, $A$ is toral.

Proof. These are mostly well-known facts or trivial consequences of them. For historical reasons, we mention justifications for Lie groups: (i) follows from (ii) (see [Gant]) and conjugacy of maximal tori (see [Hum]). For (iii), see [BoSe] (also, see [BoMo]). For (vi), see [deSieb]. We may deduce (v) from the facts that $Z(\tilde{K})$, where $\tilde{K}$ is the simply connected cover of $K$, is naturally isomorphic to $\operatorname{Hom}\left(M^{*} / M, \mathbb{K}^{\times}\right)$and that the inclusion $M \rightarrow L$ gives a natural epimorphism $\operatorname{Hom}(L, \mathbb{Z}) \rightarrow \operatorname{Hom}(M, \mathbb{Z})$. Finally, (vii) follows from (ii) and (vi). For the more general case of algebraic groups, use [SS, p. 186] for (ii); [BoLAG] for (i); [SS, pp.210-211] for (iii); same as above for (v); [St2, 2.16(c)] or [SS, p. 204] for (vi); [SS, p. 204] for (vii).

We use the system in $[\mathrm{CoGr}]$ and [CoWa] for conjugacy classes in $3 E_{6}(\mathbb{K})$, $2 E_{7}(\mathbb{K})$ and $E_{8}(\mathbb{K})$ (the setup works in this more general situation of algebraic groups) but our own for $F_{4}(\mathbb{K})$; of course, we ignore those elements listed which have orders divisible by char $\mathbb{K}$. The system is given in part in the next result and may be based on [MoPa]. In [CoGr, Table 6], the 3A centralizer should have type $A_{6} T_{1}$ and $3 \mathrm{E}$ centralizer should have type $D_{5} T_{2}$. Throughout this article, $\mathbf{p X}[G]$ shall refer to the class in $G$ labeled $\mathbf{p X}$; if confusion is unlikely, we write $\mathbf{p X}$ only and a statement $\mathbf{p X}[G]=\mathbf{p} \mathbf{X}^{\prime}[H] \cap G$ makes sense if some containment of $G$ in $H$ is understood.

(2.14) SEMISIMPLE ELEMENTS OF SMALL ORDER IN CERTAIN 
GROUPS OF LIE TYPE AND ACTION ON CERTAIN MODULES. (Eigenvalues are lifted to complex roots of unity; priming indicates distinct classes which are congruent modulo $Z(G)$ ). Class in brackets is the class of squares.

\section{TABLE VI}

\begin{tabular}{|c|c|c|c|}
\hline Group & $\begin{array}{l}\text { Class } \\
\mathbf{n X}\end{array}$ & $\begin{array}{l}\text { Eigenvalue multiplicity } \\
\text { on adjoint module } \\
\left(\text { mult }\left(\mathrm{e}^{2 \pi i k / n}\right), k=0, \ldots, n-1\right)\end{array}$ & Centralizer type \\
\hline$E_{8}(\mathbb{K})$ & $\begin{array}{l}\text { 2A } \\
\text { 2B } \\
\text { 3A } \\
\text { 3B } \\
\text { 3C } \\
\text { 3D }\end{array}$ & $\begin{array}{l}136,112 \\
120,128 \\
80,84,84 \\
86,81,81 \\
92,78,78 \\
134,57,57\end{array}$ & $\begin{array}{l}E_{7} A_{1} \\
D_{8} \\
A_{8} \\
E_{6} A_{2} \\
D_{7} T_{1} \\
E_{7} T_{1}\end{array}$ \\
\hline $2 E_{7}(\mathbb{K})$ & $\begin{array}{l}2 A \\
2 B \\
2 C=2 B^{\prime} \\
3 A \\
3 B \\
3 C \\
3 D \\
3 E \\
4 A[A] \\
4 B[B] \\
4 C[C] \\
4 D[C] \\
4 E[C] \\
4 F[B] \\
4 G[B] \\
4 H[A] \\
4 I[B] \\
4 J[C] \\
4 K[B]\end{array}$ & $\begin{array}{l}133,0 \\
69,64 \\
69,64 \\
49,42,42 \\
79,27,27 \\
43,40,40 \\
49,42,42 \\
47,43,43 \\
63,70,0,0 \\
39,30,32,32 \\
37,32,32,32 \\
33,32,36,32 \\
49,32,20,32 \\
35,32,34,32 \\
67,32,2,32 \\
79,0,54,0 \\
39,32,0,32 \\
49,32,20,32 \\
67,32,2,32\end{array}$ & $\begin{array}{l}E_{7} \\
A_{1} D_{6} \\
A_{1} D_{6} \\
A_{6} T_{1} \\
E_{6} T_{1} \\
A_{5} A_{2} \\
A_{1} D_{5} T_{1} \\
D_{5} T_{2} \\
A_{7} \\
A_{5} A_{1} T_{1} \\
A_{5} T_{2} \\
A_{3} A_{3} A_{1} \\
A_{1} D_{5} T_{1} \\
A_{1} A_{1} D_{4} T_{1} \\
D_{6} T_{1} \\
E_{6} T_{1} \\
A_{5} A_{1} T_{1} \\
A_{1} D_{5} T_{1} \\
D_{6} T_{1}\end{array}$ \\
\hline $3 E_{6}(\mathbb{K})$ & $\begin{array}{l}\mathbf{2 A} \\
\mathbf{2 B} \\
\mathbf{3 A} \\
\mathbf{3 A} \mathbf{A}^{-1} \\
\mathbf{3 B} \\
\mathbf{3 B} \\
\mathbf{3 B} \mathbf{B}^{\prime \prime}=\mathbf{3 B ^ { \prime - 1 }} \\
\mathbf{3 C} \\
\text { 3D }\end{array}$ & $\begin{array}{l}38,40 \\
46,32 \\
78,0,0 \\
78,0,0 \\
36,21,21 \\
36,21,21 \\
36,21,21 \\
24,27,27 \\
30,24,24\end{array}$ & $\begin{array}{l}A_{5} A_{1} \\
D_{5} T_{1} \\
E_{6} \\
E_{6} \\
A_{5} T_{1} \\
A_{5} T_{1} \\
A_{5} T_{1} \\
A_{2} A_{2} A_{2} \\
D_{4} T_{2}\end{array}$ \\
\hline
\end{tabular}

[The following 12 classes represent all elements of order 9 whose cube is central in $\left.3 E_{6}(\mathbb{K})\right]$

$3 \mathbf{E}$

$28,25,25$

$A_{4} A_{1} T_{1}$ 
TABLE VI (Cont.)

\begin{tabular}{|c|c|c|c|}
\hline Group & $\begin{array}{l}\text { Class } \\
\mathbf{n X}\end{array}$ & $\begin{array}{l}\text { Eigenvalue multiplicity } \\
\text { on adjoint module } \\
\left(\text { mult }\left(\mathrm{e}^{2 \pi i k / n}\right), k=0, \ldots, n-1\right)\end{array}$ & Centraizer type \\
\hline & $\mathbf{3} \mathbf{E}^{\prime}$ & $28,25,25$ & $A_{4} A_{1} T_{1}$ \\
\hline & $3 \mathrm{E}^{\prime \prime}$ & $28,25,25$ & $A_{4} A_{1} T_{1}$ \\
\hline & $(3 E)^{-1}$ & $28,25,25$ & $A_{4} A_{1} T_{1}$ \\
\hline & $\left(3 \mathbf{E}^{\prime}\right)^{-1}$ & $28,25,25$ & $A_{4} A_{1} T_{1}$ \\
\hline & $\left(3 \mathbf{E}^{\prime \prime}\right)^{-1}$ & $28,25,25$ & $A_{4} A_{1} T_{1}$ \\
\hline & $3 \mathbf{F}$ & $46,16,16$ & $D_{5} T_{1}$ \\
\hline & $\mathbf{3} \mathbf{F}^{\prime}$ & $46,16,16$ & $D_{5} T_{1}$ \\
\hline & $3 F^{\prime \prime}$ & $46,16,16$ & $D_{5} T_{1}$ \\
\hline & $(3 \mathbf{F})^{-1}$ & $46,16,16$ & $D_{5} T_{1}$ \\
\hline & $\left(3 F^{\prime}\right)^{-1}$ & $46,16,16$ & $D_{5} T_{1}$ \\
\hline & $\left(3 \mathbf{F}^{\prime \prime}\right)^{-1}$ & $46,16,16$ & $D_{5} T_{1}$ \\
\hline \multirow[t]{5}{*}{$F_{4}(\mathbb{K})$} & $2 A$ & 24,28 & $A_{1} C_{3}$ \\
\hline & 2B & 36,16 & $B_{4}$ \\
\hline & $\mathbf{3 A}$ & $22,15,15$ & $\overrightarrow{C_{3} T_{1}}$ \\
\hline & 3B & $22,15,15$ & $B_{3} T_{1}$ \\
\hline & 3C & $16,18,18$ & $A_{2} A_{2}$ \\
\hline
\end{tabular}

(2.15) DEFINITION. (Generalities about quadratic forms on $\mathbb{T}_{(n)}$ ) First, we deal with the simply laced case. Assuming $G$ is simply connected, we identify $\mathbb{T}$ with $\mathfrak{h} / L$ as usual by the epimorphism exp: $\mathfrak{h} \rightarrow \mathbb{T}$ (if $\mathbb{K}=\mathbb{C}$, this is ordinary exponentiation on a suitable module, $V$ ), where $\mathfrak{h}$ is a Cartan subalgebra in $\mathrm{g}$, the Lie algebra of $G, L$ is the root lattices (it is dual to the lattice of weights for $V$ ); $L$ is integral. Let $W$ be the Weyl group and suppose $n$ is not divisible by $\operatorname{char}(\mathbb{K})$. Due to this integrality, we define a $W$-invariant form on $\mathbb{T}_{(n)}$ as follows: let $t, u \in \mathfrak{h}$ such that $n t$ and $n u \in L$ and set $(\exp (t)$, $\exp (u))=n^{2} t \cdot u+n \mathbb{Z} \in \mathbb{Z} / n \mathbb{Z}$ (where the dot indicates the natural bilinear form on $\mathbb{K} \otimes L)$. We call this form the natural bilinear form on $\mathbb{J}_{(n)}$. When $n$ is a prime, we use the usual language of bilinear forms on finite-dimensional vector spaces to talk about structures on $\mathbb{T}_{(n)}$, the orthogonal action of the Weyl group $N(\mathbb{T}) / \mathbb{T}$ on $\mathbb{T}_{(n)}$, etc.

For the nonsimply laced case, we take a realization of our simply connected group as the fixed points of a graph automorphism of $H$, a group with a simply laced root system, then restrict the above bilinear form on a torus of $H$ to a torus of $G$.

(2.16) LEMMA. (Quadratic structure of certain $\mathbb{T}_{(n)}$; see (2.14) for notation.) (i) Let $G=F_{4}(\mathbb{K})$. Then $F:=\mathbb{T}_{(2)}$ has the following structure: a 2-dimensional 2B-pure subspace, $V_{0}$ with all other elements in $V$ of type $\mathbf{2 A}$. As modules for the 
Weyl group, $V_{0}$ and $V / V_{0}$ are not isomorphic but each get the natural action of $\mathrm{GL}(2,2)$. The Weyl group is transitive on $V \backslash V_{0}$. We have $\mathbf{2 A}\left[F_{4}\right]=$ $\mathbf{2 A}\left[3 E_{6}\right] \cap F_{4}(\mathbb{K})$ and $\mathbf{2 B}\left[F_{4}\right]=\mathbf{2 B}\left[3 E_{6}\right] \cap F_{4}(\mathbb{K})$.

(ii) Let $G$ be the group $E_{8}(\mathbb{K})$. The natural form on $\mathbb{T}_{(2)}$ carries a nonsingular W-invariant quadratic form of dimension 8 and maximal Witt index. Class $2 \mathbf{A}$, 2B corresponds to anisotropic, isotropic vectors, respectively.

(iii) Let $G$ be the group $3 E_{6}(\mathbb{K})$. The natural form on $T_{(2)}$ carries a Winvariant quadratic form of dimension 6 and nonmaximal Witt index. Class 2A, 2B corresponds to anisotropic, isotropic vectors, respectively (given an embedding of $3 E(\mathbb{K})$ in $E_{8}(\mathbb{K})$, we get $\mathbf{2 A}\left[E_{8}\right] \cap 3 E_{6}(\mathbb{K})=\mathbf{2} \mathbf{A}\left[3 E_{6}\right]$ and similarly for 2B). Since the center of $3 E_{6}(\mathbb{K})$ has order prime to $2, \mathbb{T}_{(2)}$ maps isomorphically to its image modulo the center and we may label the two classes of involutions in $E_{6}(\mathbb{K})$ with $2 \mathrm{~A}$ and $\left.\mathbf{2 B}\right)$.

(iv) Let $G$ be the group $2 E_{7}(\mathbb{K})$. The natural form on $\mathbb{T}_{(2)}$ carries a $W$ invariant quadratic form of dimension 7 . The radical of the quadratic form is trivial and the radical of the corresponding bilinear form is $Z(G) \cong \mathbb{Z}_{2}$. The center is generated by an involution from the class $\mathbf{2 A}$ in $2 E_{7}(\mathbb{R})$ (given any embedding of $2 E_{7}(\mathbb{K})$ in $E_{8}(\mathbb{K})$, this involution will be in the $E_{8}(\mathbb{K})$-class $\left.2 \mathbf{A}\right)$. Class $\mathbf{2 B}, \mathbf{2 C}$ corresponds to anisotropic, isotropic vectors, respectively (they lie in respective $E_{8}(\mathbb{K})$-classes $2 \mathbf{A}$ and $2 \mathbf{B}$ ).

Proof. In the $E_{8}$ case, the quadratic form on $T_{(2)}$ is nonsingular and has maximal Witt index since the $E_{8}$-lattice contains the $A_{2}^{4}$-lattice with odd index. The rest follows from use of (2.14) and elementary properties of quadratic forms. Notice that an $A_{2}$-lattice modulo 2 gives a quadratic form of nonmaximal Witt index. Since an $A_{2} E_{6}$-lattice has odd index in an $E_{8}$-lattice, an $E_{6}$-lattice modulo 2 has a quadratic form of nonmaximal Witt index.

(2.17) THEOREM. Up to conjugacy, $G=E_{8}(\mathbb{K})$ has two maximal elementary abelian subgroups, represented by $E$ and $F$. They have the following properties:

TYPE 1. $E=E_{1}\langle\theta\rangle$, where $E_{1}=\mathbb{T}_{(2)}$ and $\theta$ is a Chevalley involution (2.23) inverting that torus. We have $N(E) / E \cong W_{E_{8}}$ and every element of $E \backslash E_{1}$ is in $\mathbf{2 B}$.

TYPE 2. $F=F_{1} \times F_{2}$ and $F_{i} \leqslant H_{i}$, where $H_{1} \times H_{2} \leqslant G, H_{i}$ has type $G_{2}, F_{4}$ for $i=1,2$, respectively, and $F_{i}$ is (the unique, up to conjugacy) nontoral elementary abelian group in $H_{i}$. We have $\mathrm{rk} F_{1}=3$ and $\mathrm{rk} F_{2}=5$. The set $\{1\} \cup\left(\mathbf{2 B} \cap F_{2}\right)$ is a subgroup of order 4 , called $\operatorname{rad}(F)$ or $\operatorname{soc}(F)$, the radical or socle of $F$. The groups $F_{2}$ and $F_{1} \operatorname{rad}(F)$ (each of order 32) are the 2Acomponents of $F$ and they have the property that $2 \mathbf{A} \cap F$ is the disjoint union of $X \backslash \operatorname{rad}(F)$, where $X$ ranges over the two components. We have $C(F)=F$ and $N(F) / F \cong 2^{2 \cdot 3+2 \cdot 3} \cdot\left[(\mathrm{GL}(3,2)\right.$ wr 2$\left.) \times \Sigma_{3}\right]$.

Proof. [Ad]; we give another proof in Appendix 1. 
(2.18) PROPOSITION. (Conjugacy classes of outer automorphisms of order $p$ and fixed point subgroups on $G / Z, G$ simply connected almost simple; here $p=2$ with an exception of $p=3$ for $D_{4}$ and in all cases, $p \neq$ char $\mathbb{K}$.)

$n$ odd: two classes;

if $|Z|$ odd, fixed points $\mathrm{SO}(n+1, \mathbb{K})$ or $\mathrm{Sp}(n+1, \mathbb{K} \leqslant)$;

if $|Z|$ even, fixed points $\mathrm{PO}(n+1, \mathbb{K})$ or $2 \times \mathrm{PSO}(n, \mathbb{K})$, as

$(n+1) /|Z|$ is odd or even; or $\operatorname{PSp}(n+1, \mathbb{K}) \times m$, where $m=1$ or

2 as $(n+1) /|Z|$ is odd or even.

$n$ even; one class;

then $|Z|$ is odd, fixed points $\mathrm{SO}(n+1, \mathbb{K})$;

$\left(D_{n}\right) \quad 1+[(n-1) / 2]$ classes, represented on the standard $2 n$ dimensional module by $\operatorname{diag}\left(-1^{k}, 1^{2 n-k}\right)$ for an odd integer $k \in\{1,3, \ldots, 1+2[(n-1) / 2]\} ;$ fixed points on $\operatorname{Spin}(2 n, \mathbb{K})$ are $[\operatorname{Spin}(k, \mathbb{K}) \circ \operatorname{Spin}(2 n-k, \mathbb{K})]$; fixed points on $\mathrm{SO}(2 n, \mathbb{K})$ are $\mathrm{SO}(k, \mathbb{K}) \times \mathrm{SO}(2 n-k, \mathbb{K}) \times 2 ;$ fixed points on $\operatorname{PSO}(2 n, \mathbb{K})$ are $\mathrm{SO}(k, \mathbb{K}) \times \mathrm{SO}(2 n-k, \mathbb{K})$.

$\left(D_{4}, p=3\right)$ two classes; fixed points $G_{2}(\mathbb{K})$ and $\operatorname{PSL}(3, \mathbb{K})$.

$\left(E_{6}\right) \quad$ two classes, fixed points $F_{4}$ and $\operatorname{PSp}(8, \mathbb{K})\left(\right.$ type $\left.C_{4}\right)$.

Proof. Well known (at least the components, if not the precise situation for the centers); for a 'new' proof in characteristic 0 , use Theorem 8.6 of [Kac] and some easy matrix calculations. In arbitrary characteristic, matrix calculations work, except for the case of $p=3$ for $D_{4}$ and $p=2$ for $E_{6}$, for which see [CoLiSaSe] and [GoLy, §9].

(2.19) PROPOSITION. (Projectively elementary abelian groups and tensor product decomposition for forms.) Suppose that $\operatorname{char}(K) \neq 2$ and that $V, V_{1}$ and $V_{2}$ are vector spaces having nondegenerate bilinear forms $f, f_{1}, f_{2}$ with parities $\varepsilon, \varepsilon_{1}, \varepsilon_{2}(+$ for symmetric, - for alternating). Suppose furthermore that $V=V_{1} \otimes V_{2}, f=f_{1} \otimes f_{2}, E_{i} \leqslant \operatorname{Aut}\left(f_{i}\right)$ and that $E=E_{1} \circ E_{2}$, where the $E_{i}$ are identified with their images in $G:=\operatorname{Aut}(f)$. Suppose that $E_{1} \cong 2_{\varepsilon_{1}}^{1+2 r}$ is extraspecial with $Z\left(E_{1}\right)$ acting as $\{ \pm 1\}$ on $V_{1}$, $\operatorname{dim} V_{1}=2^{r}$, that $E_{2}$ is a maximal abelian subgroup of Aut $\left(f_{2}\right)$ such that $E / Z(G)$ is elementary abelian. Set $2 n=\operatorname{dim} V=2^{r}$ s and suppose that $E_{2} \cong 4^{a} \times 2^{b}, a \in\{0,1\}$.

(i) Given $r, s, r \geqslant 1,(a, b)$ must be one of the following:

if $\left(\varepsilon_{1}, \varepsilon_{2}, \varepsilon\right)=(+++):(a, b)=(0, s)$ or, only if $s$ is even, $(1, s / 2-1)$

$(--+):(a, b)=(1, s / 2-1)$;

$(+--):(a, b)=(1, s / 2-1)$;

$(-+-):(a, b)=(0, s)$ or, only if $s$ is even, $(1, s / 2-1)$. 
Note that $E_{2}$ is toral in $\operatorname{Aut}\left(f_{2}\right)$ if $a=1$. In particular, the rank of $E / E^{\prime}$ is $2 r+s$ if $(a, b)=(0, s)$ and is $2 r+s / 2$ otherwise.

(ii) For a value of $(a, b)$ as above, the group $E / E^{\prime}$ is maximal elementary abelian in $\operatorname{Aut}(f) /\{ \pm 1\}$ iff $E_{2}$ is not of the form $2^{2}$ and $\varepsilon=+$ or $4 \times 2$ with $\varepsilon$ arbitrary, in which case $E$ is contained in a subgroup of $\operatorname{Aut}(f)$ with data $(r+1+a, 1,0,1)$.

REMARK. Given a group $E=E_{1} \circ E_{2} \leqslant \operatorname{Aut}(f)$ with $E_{1}$ extraspecial, $E_{2}$ abelian, $\Phi(E) \leqslant\{ \pm 1\}$, then there is a decomposition $V=V_{1} \otimes V_{2}$ as in the proposition; see (2.11).

Proof. (i) follows from (4.1), (5.4) and, in case $\varepsilon_{2}=+$ and $E_{2}$ is elementary abelian, the decomposition of $V_{2}$ into eigenspaces for $E_{2}$.

(ii) We shall see that $E$ is nonmaximal iff $\operatorname{rk}\left(E_{2}\right) \leqslant 2$ and $\left|E_{2}\right|>2$. Consider the possibility that $E$ is contained in a larger such group $E^{*}$; then $E_{1}<E_{1}^{*} \leqslant \operatorname{Aut}(f)$ such that $E_{1}^{*}$ is extraspecial; we may assume $E_{1}^{*} \cong 2^{1+2(r+1)}$. We have $E^{*}=E_{1}^{*} E_{2}^{*}$ for some subgroup $E_{2}^{*}$ of $E_{2}$. Then, every element of $Z\left(E^{*}\right)$ has eigenvalues with multiplicity $2^{r+1}$ and every element of $E_{2} \backslash Z\left(E^{*}\right)$ has trace 0 on $V_{2}$. Thus, if $\operatorname{rk}\left(E_{2}\right) \geqslant 3$, we get a contradiction since $E_{2}$ contains elements which act on $V_{2}$ like $\operatorname{diag}(-1,1,1,1,1, \ldots)$ or $\operatorname{diag}\left(-1^{2}, 1^{k}\right)$ for even $k \geqslant 4$ (in the case $\varepsilon_{2}=-$ ). So, $E$ nonmaximal implies that $\operatorname{rk}\left(E_{2}\right) \leqslant 2$.

If $\operatorname{rk}\left(E_{2}\right)=1, E_{2}$ is scalar (whence $s=1$ and so $E=E_{1}$ is maximal projectively elementary abelian) or a generator has eigenvalues $i$ and $-i$ of equal multiplicity, whence $E_{2}$ is nonmaximal, contradiction. If $\operatorname{rk}\left(E_{2}\right)=2$, there are two cases. If $E_{2}$ is elementary, $\varepsilon_{2}=+$ and $E_{2}$ is embeddable in $\operatorname{Dih}_{8} \leqslant \operatorname{Aut}\left(f_{2}\right)$. If $E_{2}$ has exponent 4 , we may choose generators $x$ and $y$ for $E_{2}$ such that $V_{2}$ is a multiple of the following representation for $E_{2}$ :

$$
x \mapsto \operatorname{diag}(i, i,-i,-i), \quad y \mapsto \operatorname{diag}(1,-1,1,-1) .
$$

Here, $\varepsilon_{2}= \pm$. Thus, $E_{2}$ is contained in a group $2_{\varepsilon_{2}}^{1+4}$ in $\operatorname{Aut}\left(f_{2}\right)$ and so $E$ is not maximal projectively elementary abelian.

(2.20) COROLLARY. Notation as in (2.19) with $2 n=8$. Let $Z \leqslant E \leqslant F$, where, given $r$ and $s, E$ is maximal and where $F / Z$ is a maximal elementary abelian 2-group. Then $E=F$ except in the following cases:

$$
\begin{array}{cccc} 
& \multicolumn{3}{l}{(r, s, a, b ;|F: E|):} \\
\cline { 2 - 4 }(+++): & 2202 ; 2 & 2210 ; 2 & 1411 ; 4 \\
(--+): & \cdot & 2210 ; 2 & 1411 ; 4 \\
(+--): & . & 2210 ; 2 & 1411 ; 4 \\
(-+-): & 2202 ; 2 & 2210 ; 2 & 1411 ; 4
\end{array}
$$


In all cases, $F \cong 2_{\varepsilon}^{1+6}$ is uniquely determined up to conjugacy by $C(E)$ and corresponds to a case with $(r, s)=(3,1)$.

(2.21) COROLLARY. Notation as in (2.19) and (2.20). Let $\operatorname{dim} V=8$ and let $f$ be alternating or symmetric. Then $\operatorname{Aut}(f) /\{ \pm 1\}$ has two conjugacy classes of maximal nontorals and their ranks are 5 and 6 ; they correspond to cases $(r, s, a, b)=(1,4,0,4)$ and $(3,1,0,1)$.

The following result seems to be new. It says, roughly, that if a projectively elementary abelian group in an orthogonal group has imaginary elements of both spins (2.10), it is either abelian or just barely nonabelian.

(2.22) PROPOSITION. Let $G=\operatorname{Spin}(2 n, \mathbb{K})$ with $n=2$ s even and let $E / Z(G)$ be elementary abelian. Let $Z_{0}$ be the kernel of the natural map $G \rightarrow \operatorname{SO}(2 n, \mathbb{R})$. Assume that the image of $E$ in $\mathrm{SO}(2 n, \mathbb{K})$ is not elementary abelian. Let $E^{+}, E^{-}$ denote the set of spin up, spin down imaginary elements (see (2.10)) in $E$ and let $E^{\mathrm{im}}:=E^{+} \cup E^{-}$. Then $E^{\mathrm{im}} \neq \varnothing,\left[E^{+}, E^{-}\right]=\phi$ or 1 and:

(i) $\operatorname{rk}(\Phi(E))$ is the number of $\left\{E^{+}, E^{-}\right\}$which are nonempty; also, $\Phi(E)$ is generated by the squares of elements in $E^{\mathrm{im}}$;

(ii) both $E^{+}$and $E^{-}$are nonempty and $E$ is embedded in one of the following: (ii.a) a natural Quat ${ }_{8} \times$ Quat $_{8} \times 2^{s-1}$ (corresponding to a decomposition of the standard module as $V=V_{1} \otimes V_{2} \otimes V_{3}$, where $\operatorname{dim} V_{1}=\operatorname{dim} V_{2}=2$ and $\operatorname{dim} V_{3}=n / 2$ with the bilinear form factorized $f=f_{1} \otimes f_{2} \otimes f_{3}$ with $f_{1}$ and $f_{2}$ alternating, $f_{3}$ symmetric); or

(ii.b) a natural $\mathrm{Dih}_{8} \times 2^{s}$ (corresponding to $V=V_{1} \otimes V_{2} \otimes V_{3}$ as above except with each $f_{i}$ symmetric) and there is a central element of $E$ acting as diag $\left(-1^{2 a}, 1^{2 n-2 a}\right)$ with a odd;

(iii) assume $E^{-}=\varnothing$; then $E=\left\langle E^{+}\right\rangle \times Z_{0}$ or there is $X \leqslant E$, $E^{+} \subseteq X \cong \mathrm{Dih}_{8}$ and an elementary abelian group $Y$ such that $E=X \times Y$; every element of $Y$ acts on $V$ as an involution of the form $\operatorname{diag}\left(-1^{2 a}, 1^{2 n-2 a}\right)$ with a even.

Proof. Recall from (2.9) the structure of $C_{G}(e)$ for $e$ imaginary and recall (2.18). By (2.12), $\left[E^{+}, E^{-}\right]=\varnothing$ or 1 .

(i) Since $n$ is even, (2.9) tells us that the kernels $Z_{\varepsilon}$ of the two half-spin representations are generated by $\left\langle f^{2}\right\rangle$ for $f \in E^{\varepsilon}$. Since $E$ is a 2-group, $\Phi(E)=\left\langle x^{2} \mid x \in E\right\rangle$. If both $E^{\varepsilon}$ are nonempty, (i) holds. Suppose only $E^{+}$is nonempty. Since $\exp \left(E / Z_{0}\right)=4, \operatorname{rk}(\Phi(E)) \geqslant 1$, so we suppose it is 2 and seek a contradiction. Then, as $\Phi(E)=\left\langle x^{2} \mid x \in E\right\rangle$, there is $x \in E$ with $x^{2} \notin Z_{+}$, whence $E^{-}=\varnothing$ implies that $\left\langle x^{2}\right\rangle=Z_{0}$. If $[x, f]=1,(x f)^{2}=x^{2} f^{2}$ is a generator for $Z_{-}$, whence $x f \in E^{-}$, a contradiction. So, $[x, f] \neq 1$, whence $x$ 
interchanges the two eigenspaces for $f$ in the natural representation; since $x^{2} \in Z_{0}, x$ acts as an involution with eigenvalue -1 having multiplicity $n$. Since $n$ is even, $x$ is an involution, a contradiction. So, (i) holds.

(ii) Suppose both $E^{\varepsilon}$ are nonempty. We use $\left[E^{+}, E^{-}\right]=1$ and quote (2.9). From (i), we get $\left\langle E^{\text {im }}\right\rangle=\left\langle E^{+}\right\rangle \circ\langle\bar{E}\rangle$ and $\Phi(E)=\Phi\left(E^{\mathrm{im}}\right)=\Phi\left(\left\langle E^{-}\right\rangle\right) \times \Phi\left(\left\langle E^{+}\right\rangle\right) \cong 2 \times 2$. If $E \neq\left\langle E^{\mathrm{im}}\right\rangle$, every element of $E \backslash\left\langle E^{\mathrm{im}}\right\rangle$ is an involution and so $\left\langle E^{\mathrm{im}}\right\rangle$ is of index 2 and abelian of exponent 4. Let $p \in E^{\mathrm{im}}$ and let $t \in E$ be an involution inverting $p$. By (2.9), E decomposes as $\langle p, t\rangle \times L$, where the factors come from a $\mathrm{O}(2, \mathbb{K}) \times \mathrm{O}(n, \mathbb{K})$ subgroup associated to a decomposition $V=U_{1} \otimes U_{2}$. Since $\left\langle E^{\mathrm{im}}\right\rangle=C_{E}(p)$, $E=\langle p, L\rangle$. Since $t$ inverts and centralizes $L, L$ is elementary abelian. Now (2.12) implies that (ii.b) holds.

If $E=\left\langle E^{\mathrm{im}}\right\rangle$ and some factor, say $\left\langle E^{+}\right\rangle$, contains a noncentral involution, $x$, then every element of $x\left\langle E^{-}\right\rangle$is an involution and so $\left\langle E^{-}\right\rangle$is abelian. On the other hand, if $u \in E^{-}, x f$ has order 4 , a contraction. So, no such $x$ exist. We conclude that each $\left\langle E^{\varepsilon}\right\rangle$ is isomorphic to Quat $_{8} \times 2^{r}$ or $\mathbb{Z}_{4} \times 2^{r}$, for some $r$. By (2.18), any such $\left\langle E^{\mathrm{im}}\right\rangle$ lies in a subgroup of $G$ associated to a decomposition $V_{1} \otimes V_{2} \otimes V_{3}$ as in (ii.a).

For (iii), consider whether $\left\langle E^{+}\right\rangle$equals $E$ and use (2.12).

(2.23) DEFINITION. A Chevalley involution of a finite-dimensional semisimple Lie algebra $\mathfrak{g}$ with respect to the Cartan subalgebra $\mathfrak{h}$ is an automorphism of order 2 which is -1 on $\mathfrak{h}$. This element is in the connected component of the identity in the relevant adjoint algebraic group iff -1 is in the Weyl group. When this occurs, the element may be represented by an involution in the corresponding simply connected group but may not be. If it does, we say that the connected algebraic group has a Chevalley involution. If -1 is in the Weyl group and, in addition, the simply connected group $G$ has trivial center, $G$ does have a Chevalley involution. By applying this remark to $E_{8}$ and considering the subgroup $2 A_{1} E_{7}$ of $E_{8}$, we see that $2 E_{7}$ does not have a Chevalley involution since $2 A_{1} \cong \operatorname{SL}(2, \mathbb{K} \ll)$ does not.

(2.24) LEMMA. $3 \mathrm{C}\left[F_{4}\right] \subseteq 3 \mathrm{C}\left[3 E_{6}\right] \subseteq 3 \mathrm{~B}\left[E_{8}\right]$.

Proof. Use (2.14). All three classes consist of elements whose centralizer has finite center. This condition forces the first containment and allows two possibilities $E_{8}$-classes which might contain $3 C\left[3 E_{6}\right]$. The second containment is obvious since there is no embedding of a group of type $A_{2}^{4}$ in one of type $A_{8}$.

(2.25) LEMMA. Let $G \cong \operatorname{Spin}(8, \mathbb{K})$, $\operatorname{char}(\mathbb{K}) \neq 3$, and let $\alpha$ and $\beta$ be outer automorphisms of order 3 with fixed points of type $G_{2}(\mathbb{K} \leqslant)$ and $\operatorname{PSL}(3, \mathbb{K})$, respectively; see (2.18). For $\gamma=\alpha, \beta$, let $\mathbb{T}_{\gamma}$ be a maximal torus of $C_{G}(\gamma)$ and let 
$A_{\gamma}:=\left\langle\gamma, \mathbb{T}_{\gamma(3)}\right\rangle \leqslant G:\langle\gamma\rangle$, a group of order 27 . Then $\mathbb{T}_{\alpha}$ and $\mathbb{T}_{\beta}$ are $G$-conjugate and $A_{\alpha}$ and $A_{\beta}$ are $G$-conjugate.

Proof. For each $\gamma, \mathbb{T}_{\gamma}=C\left(E_{\gamma}\right)^{0} \cap C_{G}(\gamma)$, for $E_{\gamma}:=\mathbb{T}_{\gamma(3)}$, so it suffices to prove the last statement since $E_{\gamma}=G \cap A_{\gamma}$. Let $A:=A_{\gamma}, E:=E_{\gamma}$ and let $A$ contain $m$ conjugates of $\alpha$ and $n$ conjugates of $\beta$. Then $\operatorname{dim} C_{G}(A)=2$ implies (*) $\Sigma_{x \in A} \operatorname{tr}(x)=2 \cdot 27=54$, where ' $t r$ ' denotes trace on the Lie algebra of $G$, of type $D_{4}$. On the Lie algebra of $C_{G}\left(\mathbb{T}_{\gamma}\right)^{0} / \mathbb{T}_{\gamma}, \gamma$ operates fixed point freely (since $\pi_{\gamma}$ is its connected centralizer in $\left.C_{G}(\gamma)\right)$, and so $C_{G}\left(\mathbb{T}_{\gamma}\right)^{0} / \mathbb{T}_{\gamma}$ is nilpotent. Since $C_{G}\left(\mathbb{T}_{\gamma}\right)$ is reductive, it is a torus. Therefore, $(* *) \Sigma_{x \in E} \operatorname{tr}(x)=4 \cdot 9=36$. Since $\operatorname{tr}(\alpha)=7$ and $\operatorname{tr}(\beta)=-2,(*)$ and $(* *)$ imply that $54=36+7 m-2 n$. Since $m+n=18$, we conclude that $m=6$ and $n=12$. Therefore, $G$-conjugates of $E_{\alpha}$ and $E_{\beta}$ lie in both $C_{G}(\gamma)$. This implies that they are conjugate in $C_{G}(\beta)$, since the nontoral nines group $E$ in $C_{G}(\beta)$ is self-centralizing, whence $\Sigma_{x \in\langle E, \alpha\rangle} \operatorname{tr}(x)=0 \neq 36$.

(2.26) LEMMA. Let $G \cong 2 E_{7}(\mathbb{K}), H \cong E_{8}(\mathbb{K}), K$ a $4 A_{7}$-subgroup of $G$ and $\varphi: \mathrm{SL}(8, \mathbb{K}) \rightarrow K$ an epimorphism. Suppose that $V$ is a four group in $K$ such that $V \varphi^{-1} \cong$ Quat $_{8}$. Then $V \subseteq \mathbf{2 B}[G] \subseteq \mathbf{2 A}[H]$.

Proof. Since $N_{K}(V)$ induces $\Sigma_{3}$ on $V, V$ is pure with respect to the class $2 \mathbf{B}[G]$ or $2 \mathbf{C}[G]$. We embed $V$ in a group $U \leqslant K, U \cong 2^{7}$ (so $\left.U \varphi^{-1} \cong 4 \circ 2^{1+6}\right)$. Orthogonality relations (1.5) tell us that $\operatorname{dim} C_{G}(U)=7$. If $C_{G}(U)^{0}$ is not a torus, it is reductive of type $T_{1} A_{1}^{2}$ or $T_{4} A_{1}$ and the perfect group $N_{K}(U) \cong 4 \circ 2^{6} \cdot \mathrm{Sp}(6,2)$ acts on it, forcing $N_{K}(U)$ to contain an $A_{1}$ component, a contradiction. Therefore, $C_{G}(U)^{0}$ is a torus, in $C\left(\mathrm{O}_{2}\left(N_{G}(K)\right)\right)$. Since there is $x \in N_{K}(U)$ such that $|x|=3$ and $\left[\left(C_{G}(U)^{0}\right)_{(2)}, x\right]=V, V^{\#}$ consists of nonsingular vectors in the sense of (2.16.iv) and so $V$ is $2 B[G]-$ pure, as required.

\section{Type $A$}

(3.1) THEOREM. Let $p$ be a prime, $G=\mathrm{SL}(n+1, \mathbb{K}), Z \leqslant Z(G), E$ a $p$ subgroup of $\mathrm{GL}(n+1, \mathbb{K})$ such that $E Z / Z$ is elementary abelian. If $|Z| \neq \equiv$ $(\bmod p), E$ is toral. If $|Z| \equiv 0(\bmod p)$, nontoral groups exist and maximal ones are the images $\bmod Z$ of a group of the form $A \circ B$, where $A \cong p_{+}^{1+2 r}$, for some $r \geqslant 1$ and where $B$ is an abelian (and toral) subgroup of the commuting algebra of $A, n+1=p^{r}$. Given $A$, if $B$ is as large as possible, $\operatorname{rk}(B)=s$ and $B \cong \mathbb{Z}_{p^{c+1}} \times p^{s-1}$, where $p^{c}=|Z|_{p}$. We have $A B \cap G=A B_{1}$, where $\Omega_{1}(B) \Phi(B) \leqslant B_{1} \leqslant B$ and $B_{1}=B$ iff $p^{c+1} \mid(n+1)$.

Proof. We may assume that $E$ is nonabelian, which implies that $|Z| \equiv 0$ $(\bmod p)$. Thus, $\left|E^{\prime}\right|=p$ and $(2.4)$ applies to give $E=A \circ B$ as above; the representation theory of extraspecial groups does the rest; see [Go], [Hup]. 


\section{TYPE $C$}

Let $V$ be a $2 n$-dimensional vector space over $\mathbb{K}$ and $f$ an alternating bilinear form. Let $G=\operatorname{Aut}(f)=\operatorname{Sp}(2 n, \mathbb{K})$, let $p$ be a prime and let $E$ be a $p$-subgroup of $G$ which is elementary abelian modulo $Z(G)=\{ \pm 1\}$.

(4.1) PROPOSITION. Let $p=2$. If $E$ is abelian, $E$ is toral. If $E^{\prime}=\{ \pm 1\}$, then $E$ is associated to a tensor decomposition and has isomorphism type as in (2.4.ii). If $E$ is maximal with respect to stabilizing that tensor product decomposition, the rank of $E / E^{\prime}$ is a number of the form $2 r+s-1$ or $2 r+s / 2-1$, where $2^{r} s=2 n$, according to whether $\exp (Z(E))=2$ or 4 .

Proof. Suppose that $E$ is elementary abelian. If $\lambda$ is a character of $E$ and $V_{\lambda}$ the corresponding eigenspace, $f_{\lambda}:=f \mid V_{\lambda}$ is nonsingular and so $C(E)$ is the direct product of the $\operatorname{Aut}\left(f_{\lambda}\right)$. We finish by observing that a torus of this group has dimension $n$ hence is a maximal torus of $\operatorname{Aut}(f)$.

Now, suppose that $\exp E=4$. The structure of $E$ is given by (2.4.ii). If $E$ is abelian, let $f$ be an element of order 4 . Then, $E$ is contained in a natural $\mathrm{GL}(n, \mathbb{K})$-subgroup centralizing $f$ and so $E$ is toral. For the general case, we quote (2.11) and (2.19).

(4.2) PROPOSITION. Let $p$ be odd. Every abelian p-subgroup of $\mathrm{Sp}(2 n, \mathbb{K})$ is toral.

Proof. Since $(p,|Z(G)|)=1$, we may assume $E$ is an elementary abelian subgroup of $G$. We decompose $V$ by characters of $E$. Since $p$ is odd, the eigenspaces $V_{\lambda}$ are totally singular for $\lambda \neq 1$ but $V_{\lambda^{-1}}$ is paired with $V_{\lambda}$ nonsingularly. Let $U$ be an $E$-invariant maximal isotropic subspace and $W$ an $E$-invariant maximal isotropic complementing $U$. Then, $E$ lies in the natural $\mathrm{GL}(n, \mathbb{K})$-subgroup of $G$ stabilizing the decomposition $V=U \oplus W$, and we get $E$ toral by quoting (3.1).

\section{TyPES $B D$}

Here, $G$ is the group $\operatorname{Spin}(m, \mathbb{K})$. The center is $\mathbb{Z}_{2}, \mathbb{Z}_{2} \times \mathbb{Z}_{2}, \mathbb{Z}_{4}$ as $m$ is odd, $\equiv 0(\bmod 4), 2(\bmod 4)$, respectively. Let $n$ be the rank, so that $m=2 n$ or $2 n+1$. The difficulty here is sorting out when we get abelian images of a projectively abelian group $E$ modulo various subgroups of $Z(G)$.

(5.1) NOTATION. We begin by studying the case $p=2 \neq$ char $\mathbb{K}$. We assume that the image of $E$ in $\mathrm{SO}(m, \mathbb{K})$ is elementary abelian, whence $E$ leaves invariant the signed frame for an orthonormal basis $e_{1}, \ldots, e_{m}$ on $V$, the standard module. We use terminology from (2.7). Let $\varphi_{i}$ be the character on $E$ afforded by $\mathbb{R} e_{i}$ and for a sequence of indices $i, j, \ldots$, let $\varphi_{i j \ldots}$ be $\varphi_{i} \varphi_{j} \ldots$. Let $\varepsilon_{i}$ 
be the reflection at $e_{i}, D$ the diagonal frame group of order $2^{m}$ generated by these $\varepsilon_{i}$ and $D_{0}:=\{d \in D \mid \operatorname{det}(d)=1\}$. We let $Q, Q_{0}$ be the respective preimages of $D, D_{0}$ in $\operatorname{pin}(m, \mathbb{K})$, $\operatorname{Spin}(m, \mathbb{K})$; see $(2.8)$.

(5.2) LEMMA. Assume (5.1). Equivalent are: (i) With the exception of at most one $i$, each $\varphi_{i}$ occurs with even multiplicity; (ii) $E$ stabilizes a maximal totally isotropic subspace; (iii) $E$ is in a torus.

Proof. We prove cyclic implications. Assume (i). If $e_{r}$ and $e_{s}$ afford the same $\varphi_{l}, e_{r} \pm i e_{s}\left(i^{2}=-1\right)$ are isotropic; (ii) follows by induction. If $E$ stabilizes a maximal totally isotropic subspace, $I$, it stabilizes a maximal totally isotropic subspace $J$ such that $I \cap J=0$. Therefore, $\operatorname{dim} V / I+J \leqslant 1$ and $E$ lies in the natural $\mathrm{GL}(n, \mathbb{K})$-subgroup stabilizing both $I$ and $J$, whence (iii). Finally, if $E$ is toral, it stabilizes maximal isotropic subspaces $I$ and $J$ with $I \cap J=0$. Since $I$ and $J$ are dual $E$-modules, they are isomorphic and so (i) follows.

(5.3) EXAMPLE. An abelian $E$ may have small rank relative to the Lie rank of $G$, yet be nontoral. Suppose $m=2^{k}, k \geqslant 2$. Then, identifying the index set $\Omega$ with $\mathbb{F}_{2}^{k}$, we get the rank $k$ group $E$ consisting of the identity and all those elements of $D$ (see (5.1)) which negate at an index set corresponding to the complement of a codimension 1 linear subspace; thus, $E$ may be naturally identified with $\operatorname{Hom}\left(\mathbb{F}_{2}^{k}, \mathbb{F}_{2}\right)$. Given two distinct points of $\mathbb{F}_{2}^{k}$, there is a hyperplane which separates them. Using (5.2), we conclude that $E$ is not toral; in fact, the centralizer of the image of $E$ in the orthogonal group is the diagonal frame group. Every maximal subgroup of $E$ is toral; for, if $F$ is such, $F$ is the annihilator in $\operatorname{Hom}\left(\mathbb{F}_{2}^{k}, \mathbb{F}_{2}\right)$ of a nonzero vector $v \in \Omega$, and we use (5.2) with the observation that $e$ and $e+v$ afford the same characters of $F$, for $e \in \Omega$. There are examples of nonabelian $E$ of order 8 whose image in the orthogonal group is a fours-group, e.g. a quaternion subgroup of a natural $\mathrm{SO}(4, \mathbb{K}) \cong \mathrm{SL}(2, \mathbb{K}) \times \mathrm{SL}(2, \mathbb{K})$.

(5.4) LEMMA. Assume $E$ is abelian. (i) If $E$ has an imaginary element (see (2.10)) then, $E$ is toral. (ii) If $E$ is maximal elementary abelian, the image of $E$ in $\mathrm{SO}(\mathrm{m}, \mathbb{K})$ stabilizes a frame and corresponds to a weakly self-orthogonal code in the frame group.

Remark. The codes in (ii) are not explicitly known, except for small ranks; see (2.2) and (2.3) for ranks up to 10.

Proof. (i) The image of $E$ is embedded in the natural GL( $n, \mathbb{K})$-subgroup which stabilizes both eigenspaces for $f$ on $V$, where $f \in E$ is imaginary. Therefore, the image of $E$ is toral and so is $E$ itself.

(ii) Here, (5.1) applies. Since $E$ is elementary abelian, $E$ corresponds to a doubly even code (2.1) in the frame group. 
(5.5) LEMMA. Assume $E$ is nonabelian. Then, $E$ is in the preimage of a frame group and we are in a tensor product situation in the sense of (1.2) and (2.19).

Proof. Let $Z_{0}$ be the kernel of the action of $G$ on the natural module. If $E / Z_{0}$ is elementary abelian, it is in a frame group. Suppose $E / Z_{0}$ is nonabelian. Then (2.4) applies to show that we are in a tp-situation.

The preceding results cover all possibilities for $Z$ and show that on the natural module $E$ stabilizes a frame, a maximal isotropic subspace or is a tpgroup in the sense of (1.2). To analyze the possibilities for $\Phi(E)$ and $E^{\prime}$, see (2.22).

(5.6) EXAMPLES. (i) We remark on the situation with $\mathrm{SO}(4, \mathbb{K})$, whose simply connected covering is $\operatorname{Spin}(4, \mathbb{K}) \cong \operatorname{SL}(2, \mathbb{K}) \times \operatorname{SL}(2, \mathbb{K})$, with the factors corresponding to a tensor decomposition of $V$. If we let $Q_{i}$ be a quaternion group of order 8 in the $i$ th factor, $i=1,2$, and set $Q:=Q_{1} \times Q_{2}$. The image of $Q$ in $\mathrm{SO}(4, \mathbb{K})$ is $2_{+}^{1+4}$ and the image in a half-spin representation is quaternion of order 8 . If $n \geqslant 3$, the kernel of the action of $\operatorname{Spin}(4, \mathbb{K})$ on one of the half-spin modules for $\operatorname{Spin}(2 n, \mathbb{K})$ is just the group of order 2 in the center of one of the factors of $\operatorname{Spin}(4, \mathbb{K})$.

(ii) We look at $\mathrm{SO}(8, \mathbb{K})$. The group $\mathrm{O}(8, \mathbb{K})$ contains $W_{E_{8}} \geqslant W_{D_{7}} \cong 2^{7}: \Sigma_{8}$ and the intersection of the latter group with $\mathrm{SO}(8, \mathbb{K})$ is $L \cong 2^{7}$. $\mathrm{Alt}_{8}$. The preimage of $L$ in $\operatorname{Spin}(8, \mathbb{K})$ is $M \cong\left[2_{+}^{1+6} \times 2\right] \cdot \mathrm{Alt}_{8}$ and the image of $M$ in either one of the half-spin groups is of the form $2_{+}^{1+6} \cdot \mathrm{Alt}_{8}$ (the two images are isomorphic, via conjugation in $\operatorname{pin}(\mathbb{K})$ by preimages of determinant -1 elements of $2^{7}: \Sigma_{8}$ ).

(5.7) REMARK. The case of odd primes is handled as in (4.2).

\section{TYPE $G_{2}$}

(6.1) THEOREM. There is a unique conjugacy class of elementary abelian 2groups of order $2^{n}$, for $n=1,2,3$. In particular, such a subgroup is toral iff its rank is at most 2. For odd p, every abelian p-subgroup is toral.

Proof. An easy analysis, using (2.13.vi). Note that a four-group must be toral with centralizer $\mathbb{T}_{2}: 2$ and that, for odd $p$, a group of type $p \times p$ must be toral with centralizer $\mathbb{T}_{2}$.

\section{TYPE $F_{4}$}

We first deal with the prime 2 . We recall some information from (2.14). There are two classes of involutions, denoted $\mathbf{2 A}$ and $\mathbf{2 B}$, with centralizers of respective shapes $C_{3} A_{1}$ and $B_{4}$; in both cases, the center has order 2 and in the first case, each factor has center of order 2 . 
(7.1) NOTATION. We define complexity (abbreviated cx; see (1.3)) with respect to the class $2 \mathrm{~A}$.

(7.2) NOTATION. We use the orthogonal frame notation of (2.7) for a $\operatorname{Spin}(9, \mathbb{K})$-subgroup of $F_{4}(\mathbb{K})$ and write $x \in D$ as a vector with entries \pm 1 , indexed by $\{1, \ldots, 9\}$, with \pm 1 at $i$ iff $e_{i} x= \pm e_{i}$, where the $e_{i}$ are the vectors of a frame.

(7.3) THEOREM. Let $p=2$ and $G=F_{4}(\mathbb{K})$. Then:

(i) The elementary abelian subgroup $E$ is toral iff $\operatorname{cx}(E) \leqslant 2$. In any case, $\operatorname{cx}(E) \leqslant 3$. In particular, $E$ is nontoral iff $E$ contains a $2 \mathrm{~A}$-pure eights group.

(ii) There is, up to conjugacy, a unique maximal elementary abelian 2group in $G$, represented, say, by $M:=\mathbb{T}_{(2)}\langle\theta\rangle$, where $\theta$ is an involution in $N(\mathbb{T})$ inducing -1 on $\mathbb{T}$; see (2.23). Then, $M$ has rank 5 and there is a subgroup $N$ of order $4($ written $N=\operatorname{rad}(M)=\operatorname{soc}(M))$ such that $M \backslash N=M \cap \mathbf{2 A}$ and $M \cap \mathbf{2 B}=N^{\#}$. Also, $M=C(M)$ and $N(M) / M \cong$ $2^{2 \cdot 3}:\left(\mathrm{GL}(3,2) \times \Sigma_{3}\right)$.

Proof. (i) The only-if part follows from (2.16.i). Now let $E$ have complexity at least 3 and normalize $\mathbb{T}_{(2)}$. Then $E$ centralizes $z \in \mathbb{T}_{(2)} \cap \mathbf{2 B}$ and so lies in $C(z) \cong \operatorname{Spin}(9, \mathbb{K})$. By $(2.8)$, we may assume that the image of $E$ in $\mathrm{SO}(9, \mathbb{K})$ is a subgroup of $D_{0}$ consisting of elements of types $\left(-1^{8}, 1^{1}\right),\left(-1^{4}, 1^{5}\right)$ and $\left(1^{9}\right)$ only. By (2.2), there is a unique maximal such subgroup under the natural action of $\Sigma_{9}$ on the nine $e_{i}$ 's; a representative is $F$, which is, in the notation of (7.2), generated by $x_{0}:=(=1,-1,-1,-1,-1,-1,-1,-1 ; 1), x_{1}:=(-1$, $-1,-1,-1,1,1,1,1 ; 1), x_{2}:=(-1,-1,1,1,-1,-1,1,1 ; 1), x_{3}:=(-1,1$, $-1,1,-1,1,-1,1 ; 1)$. In fact, this space may be viewed as the famous extended Hamming code, (2.3), with an extra trivial coordinate; its group is the affine group $\operatorname{AGL}(3,2) \cong 2^{3}: \operatorname{GL}(3,2)$. Let $y \in C(z)$ map to $x_{0}$. We have $C(y, z) \cong \operatorname{Spin}(8, \mathbb{K})$ and so $y \in \mathbf{2 B}$. If $u$ is a lift of one of the other $x_{i}, u \notin \mathbf{2 B}$, or else $E$ is a rank $5 \mathbf{2 B}$-pure subgroup of $G$; we get a contradiction because, on one hand, the orthogonality relations $(1.5)$ imply that $\operatorname{dim} C_{H}(E)=0$ (where $\left.G \leqslant H \cong E_{8}(\mathbb{K})\right)$ while on the other hand, $G \times L$ is embedded in $H$, where $L \cong G_{2}(\mathbb{K})$. It is now clear that an element of $F$ lifts to an element of $2 B$ iff it is in the span of $x_{0}$ and that a subspace of $F$ lifts to a subgroup of $G$ of complexity at most 2 iff its image in $F /\left\langle x_{0}\right\rangle$ has dimension at most 2.

The statement about the normalizer and centralizer may be deduced from the structure of $\operatorname{Spin}(9, \mathbb{K})$ and the embedding of $M$ in $C(x)$, for any element $x \in \mathbf{2 B} \cap M$.

(ii) follows from the analysis in (i), together with the fact that the 'Chevalley involution' (2.23) is available in $G$. 
(7.4) THEOREM. Let $p$ be an odd prime and $G=F_{4}(\mathbb{K})$. Every elementary abelian p-subgroup of $G$ is toral, except for a single class of groups of order 27. $A$ representative $E$ of this class satisfies: $C(E)=E$ and $N(E) \cong 3^{3}: \operatorname{SL}(3,3)$.

Proof. A p-group in $G$ is total if $p \geqslant 5$ since the Weyl group has order $2^{7} 3^{2}$. We take $p=3$ and consult (2.14). There are three classes of elements of order 3 , with respective centralizers $3 A_{2} A_{2}, \mathbb{T}_{1} B_{3}$ and $\pi_{1} C_{3}$; the first is a nontrivial central product over $\mathbb{Z}_{3}$. On the 52-dimensional adjoint module, the traces are $-2,7$ and 7 , respectively.

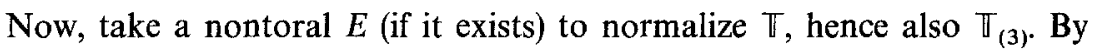
using (2.14), we get that the above classes meet $\mathbb{T}_{(3)}$ in sets of 32,24, 24 elements, respectively. It follows that $E$ centralizes some $z$ from the first class, and so $E$ is in a copy of $X:=X_{1} X_{2}$, where $X_{1} \cong X_{2} \cong \mathrm{SL}(3, \mathbb{K})$ and $X_{1} \cap X_{2}=Z:=Z\left(X_{i}\right), i=1,2$. Let $Q_{i}$ be the quasiprojections (see (2.5)) of $E$ to $X_{i}, i=1,2$, and set $Q:=Q_{1} Q_{2}$. Thus, $Q_{i}$ is abelian or extraspecial. If $Q_{i}$ is abelian, for some $i$, it is abelian for both $i$, whence $E$ is toral, by (3.1). So, we may assume that each $Q_{i}$, hence also $Q$, is extraspecial. In particular, $E \cap Q_{i}=Z$, for $i=1,2$. Note that $\exp (Q)=3$; for otherwise, some $\exp \left(Q_{i}\right)=9$, whereas $3^{1+2}$ does not embed in $\operatorname{SL}(3, \mathbb{K})$. We have $N_{X}(Q) \cong 3^{1+2} \operatorname{SL}(2,3) \circ 3^{1+2} \operatorname{SL}(2,3)$ and there is one orbit on maximal abelian subgroups which meet $X_{i}$ trivially, $i=1$ and 2 . Let $E$ be such a subgroup. Then $C(E)=E$ and $N_{X}(E)$ induces on $E$ the group $3^{2}: \operatorname{SL}(2,3)$, stabilizing a 1-space. Since $C(E)$ is 0 -dimensional, the sum of traces (in the sense of (1.5)) is 0 , whence every element of $E^{\#}$ has trace -2 , and so all elements of $E^{\#}$ are conjugate. By embedding $E$ in $C(x)$, for all $x \in E^{\#}$, we get that $C(E)=E$ and $N(E) / E \cong \mathrm{SL}(3,3)$. The extension splits since $N_{X}(E)$ splits over $E$ and its index in $N(E)$ is prime to 3; alternatively, one may quote the result that $H^{2}\left(\mathrm{SL}(3,3), 3^{3}\right)=0$ ([Sah], [Gr1]).

(7.5) REMARK. Another proof of splitting uses the lifting criterion (see Appendix 2) and the fact that the graph automorphism of $F_{4}\left(2^{r}\right)$ may be chosen to normalize an analogue of $E$ and induce -1 on it, hence centralize an $\operatorname{SL}(3,3)$ subgroup in the normalizer. The graph automorphism is available only in characteristic 2 , hence the possible need to shift characteristic. This observation gives a proof that $\operatorname{SL}(3,3)$ is in ${ }^{2} F_{4}(2)^{\prime}$, an embedding proved by K. C. Young by computer [Yo].

\section{TYPE $E_{6}, p=2$}

Recall from (2.14) that there are two classes of involutions, called $\mathbf{2 A}$ and $\mathbf{2 B}$, with respective centralizers of shapes $2 A_{5} A_{1}$ and $\mathbb{T}_{1} D_{5}$; each simple factor is simply connected and the center of the $\mathbf{2 A}$-centralizer is cyclic of order 6 (see 
(2.13.iv)), while the center of the $\mathbf{2 B}$-centralizer is $\mathbb{T}_{1}$ (otherwise, the center contains a four-group and we contradict (2.14) and (1.5)).

(8.1) NOTATION. We use the notion of complexity based on class $2 \mathrm{~A}$. Let $G=3 E_{6}(\mathbb{K})$ be embedded in $E_{8}(\mathbb{K})$. We have $2 \mathrm{~A}\left[3 E_{6}(\mathbb{K})\right]=$ $2 \mathrm{~A}\left[E_{8}(\mathbb{K})\right] \cap 3 E_{6}(\mathbb{K})$.

(8.2) THEOREM. E is toral iff its complexity is at most 2; in any case, the complexity is at most 3 and complexity 3 occurs. There is a unique maximal nontoral group, and it has rank 5 . It lies in a natural $F_{4}(\mathbb{K})$-subgroup.

Proof. We take $E$ to normalize $\mathbb{T}$, thus centralizing some $z \in \mathbb{T} \cap$ 2B. Thus, $E \leqslant C(z)$ of type $D_{5} T_{1}$.

The classification of maximal elementary abelian subgroups of this group follows from (2.2) and (2.8), using the fact that the quasiprojections are abelian (though not elementary abelian) and the facts that the nontoral group is self-centralizing in the $D_{5}$-factor and that the toral group has centralizer $\mathbb{T}_{5}$. We conclude that, in $G$, there are two and that they have ranks 5 and 6 ; from (5.2) and (2.16.iii), we get that one is toral (rank 6) and the other (rank 5) is not. The nontoral one is conjugate to a nontoral rank 5 group in a natural $F_{4}(\mathbb{K})$-subgroup; use (2.16.i) and (7.3) and notice the class distribution.

The complexity assertion follows from the corresponding one for $E_{8}(\mathbb{K})$, which will be proved in (9.4). Here is the argument. Let $E$ be a nontoral elementary abelian 2-group in $G$. Then, $E$ is nontoral in $K$, a natural $F_{4}(\mathbb{K})$ subgroup. Since, by (7.3.i) applied to $K, E$ contains a $2 \mathrm{~A}$-pure eights-group, the $E_{8}(\mathbb{K})$-complexity is 3 , so the $G$-complexity is 3 .

(8.3) REMARK. The group $E$ from the last paragraph lies in a natural $\left(G_{2} \times F_{4}\right)$-subgroup of $E_{8}$. So, we take an appropriate $A_{2}$ in the $F_{4}$-factor and look at its centralizer. It is $G_{2} \times A_{2}$ (look at a Sylow 3-normalizer in $W_{F_{4}}$ to see this). Thus, our group $E$ is of the form $E_{1} \times E_{2}$, where $E_{1}$ lies in the $G_{2}$-factor and $E_{2}$ lies in the $3 A_{2}$-factor and is a four-group in a natural $\operatorname{SO}(3, \mathbb{K})$ subgroup.

(8.4) REMARK. An elementary abelian 2-group $E \leqslant 3 E_{6}(\mathbb{K})$ is toral in $3 E_{6}(\mathbb{K})$ iff it is toral in $E_{8}(\mathbb{K})$; this is due to the compatibility of the complexity criteria; see (8.2) and (9.2).

\section{TyPES $E_{8}$ AND $E_{7}, p=2$}

It seems easier to treat $E_{7}$ after $E_{8}$, for which the result (2.17) of J. F. Adams may be quoted; recall the terms type 1 and type 2 for the two (up to conjugacy) maximal elementary abelian subgroups in $G=E_{8}(\mathbb{K})$. 
Recall from (2.14) that in $G$, there are two classes of involutions, $\mathbf{2 A}$ and $\mathbf{2 B}$, with respective centralizers $2 A_{1} E_{7}$ and $H \operatorname{Spin}(16, \mathbb{K})$; in the first centralizer, both factors are simply connected. We use the notion of complexity (1.3) based on the class $\mathbf{2 A}$.

(9.1) LEMMA. (i) If $E=E_{1} \times E_{2}$, where the $E_{i}$ are four-groups and $E \cap \mathbf{2 A}=E_{1}^{\#} \cup E_{2}^{\#}$, then $E$ is toral, unique up to conjugacy and has centralizer $C(E)=\left[\mathbb{T}_{4} \times \mathrm{SL}(3, \mathbb{K}) \times \mathrm{SL}(3, \mathbb{K})\right] .2^{2}$ (with one outer involution switching the factors and another inducing the graph automorphism on both and inverting the torus).

(ii) If, for $i=1,2,3, E_{i}$ is a four-group, $E=E_{1} \times E_{2} \times E_{3}$ and if, for any pair $i \neq j,\left(E_{i} \times E_{j}\right) \cap \mathbf{2 A}=E_{i}^{\#} \cup E_{j}^{\#}$, we have $C(E)=\left[\mathbb{\pi}_{6} \times \mathrm{SL}(3, \mathbb{K})\right]: 2$, where an involution outside $C(E)^{0}$ inverts the torus factor and induces the graph automorphism on the $\operatorname{SL}(3, \mathbb{K})$-factor.

Proof. (i) This is proved by an easy extension of the arguments in (3.7) of [CoGr]. It helps to notice that the global stabilizer of $E$ in the Weyl group is of the form $\langle-1\rangle \times\left(\Sigma_{3}\right.$ wr 2$) \times\left(\Sigma_{3}\right.$ wr 2$)$. We remark that the Chevalley involution (2.23) is in $G$. The proof of (ii) is similar in spirit.

(9.2) THEOREM. E has complexity at most 3 and, unless $E$ is eccentric, i.e. a 2B-pure subgroup of rank 5 (which is nontoral), the following holds:

$E$ is toral iff the complexity of $E$ is at most 2.

Furthermore, both type 1 and type 2 have complexity 3.

Proof. We first prove that $E$ has complexity at most 3 . Suppose that $E$ has type 1. Without loss, $E$ contains $\mathbb{T}_{(2)}$ with index 2 . Let $\kappa$ be the characteristic function of $2 \mathrm{~A} \cap E$ and let $\varphi$ be the linear functional on $E$ with kernel $\mathbb{T}_{(2)}$. Let $q$ be the natural quadratic function on $\mathbb{T}_{(2)}$, extended to any quadratic function, $r$, on $E$. Then $\kappa=(1+\varphi) r$, whence $\kappa$ has degree at most 3. It is obviously not quadratic, so its degree as a polynomial function is exactly 3 . Thus, complexity 3 occurs in $G$, as claimed.

Next, suppose that $E$ has type 2. Then, taking $E=F_{1} \times F_{2}$ as in (2.16), we refer to (2.16) and (7.3) to get a rank 2 subgroup $F_{3}$ of $F_{2}$ which is 2B-pure, i.e. the radical of $E$. We have $F_{2} \cap \mathbf{2 B}=\mathbf{F}_{3}^{\#}$ and $F_{1} F_{3} \cap \mathbf{2 B}=F_{3}^{\#}$ and $E \cap \mathbf{2 A} \subseteq F_{1} F_{3} \cup F_{2}$. Thus, $\kappa$ is the lift to $E$ of a function on $F_{1} \times F_{2} / F_{3}$, which is clearly the sum of two functions $f_{1}$ and $f_{2}$, with respective 0 -sets $F_{1}$ and $F_{2} / F_{3}$; such functions are cubic and $f_{1}+f_{2}$ is cubic since $\operatorname{deg}\left(f_{1}+f_{3}\right) \leqslant \operatorname{deg}\left(f_{i}\right)=3, i=1,2$, and its restriction to $F_{1}$ is $f_{2}$, which is cubic.

We now determine the relation between complexity and torality. If $E$ is toral, $\operatorname{cx}(E) \leqslant 2$ since $\kappa$ is just the restriction of $q$, given an embedding of $E$ into $\pi_{(2)}$; see (2.16). 
Suppose $\operatorname{cx}(E) \leqslant 2$. By (2.13.vii), we may assume $E$ has rank at least 3 . We consider two cases. We use the notation $\sigma \kappa(E):=\Sigma_{a \in E} \kappa(a)$.

CASE $1 . E$ is in a maximal elementary abelian group $M$ of type 1 . Replace $M$ by a conjugate to assume that $\mathbb{T}_{(2)}$ is in $M$. If $E$ is not in $\mathbb{T}_{(2)}, F:=\mathbb{T}_{(2)} \cap E$ has codimension 1 in $E$, hence dimension at least 2 . Since $\kappa$ is 1 on $M \backslash \pi_{(2)}$, $\sigma \kappa(E)=\sigma \kappa(F)$.

If $F$ contains a nonsingular 2-dimensional subspace, $U$, then $\sigma \kappa(U) \neq 0$ since $|U \cap \mathbf{2 A}|=1$ or 3. Taking $W:=\langle U, e\rangle$, for some $e \in E \backslash \mathbb{\mathbb { T }}_{(2)}$, we get $\sigma \kappa(W)=\sigma \kappa(U) \neq 0$, in contradiction to $\mathrm{cx}(E) \leqslant 2$. Therefore, $F$ has no such subspace.

If $F$ is 2B-pure, then so is $E$ and we quote [(3.8), CoGr].

Finally, we may assume that $F$ has a codimension 1 subspace $U$ which is 2B-pure such that $F \backslash U \subseteq \mathbf{2 A}$; then $\operatorname{rk}(F) \leqslant 4$. Properties of the quadratic form on $T_{(2)}$ allow us to enlarge $E$ if necessary to make $\operatorname{dim} U=3$; we retain $\operatorname{cx}(E) \leqslant 2$. Then $C(U)$ has shape $2^{4} A_{1}(\mathbb{K})^{8} \cdot 2^{3}$ and $C(F)$ has shape $2^{4} A_{1}(\mathbb{K})^{8}$. It follows that $E$ lies in a torus of $C(F)$ since $E / F$ is cyclic. So, $E$ is toral in $G$.

CASE 2. $E$ is in a maximal elementary abelian group $M$ of type 2. Using above notation, $F_{3}=\operatorname{rad}(M)$, where $M=F_{4} F_{2}$ and $F_{4}=F_{1} F_{3}$, i.e. $F_{2}$ and $F_{4}$ are the two $2 \mathbf{A}$-components; see (2.17). Recall that $\kappa$ is constant on cosets of $F_{3}$; so, without loss, $E \geqslant F_{3}$. Then, $\operatorname{cx}(E) \leqslant 2$ implies that $E_{k}:=E \cap F_{k}$ has rank $r_{k}$ at most $4, k=2,4$. It follows that rk $E \leqslant 7$. If $E$ is $2 \mathrm{~B}$-pure, we quote [(3.8), CoGr]. We may therefore assume $E$ is not $\mathbf{2 B}$-pure. Let $p_{i}$ be the projection of $M$ onto $M / F_{j}$, for $\{i, j\}=\{2,4\}$ and let $d_{i}:=\operatorname{dim} p_{i}(E) \geqslant r_{i}-2$.

If $E \cap \mathbf{2 B} \subseteq F_{3}$, there is $j \in\{2,4\}$ such that $E \subseteq F_{j}$ and we are done because $F_{j}$ lies in a natural $F_{4}(\mathbb{K})$-subgroup, for which the complexity criterion is compatible with overgroups $3 E_{6}(\mathbb{K})$ and $E_{8}(\mathbb{K})$; see also (2.13.i). We conclude that $E$ contains 'diagonal elements' $e=e^{\prime} e^{\prime \prime}$ with $e^{\prime} \in F_{2} \backslash F_{3}$ and $e^{\prime \prime} \in F_{4} \backslash F_{3}$. We have that $d_{i} \geqslant 1$ for $i=1,2$ (see the previous paragraph).

Subcase 2.1. Suppose that $E$ contains a four-group $U$ of diagonal elements (i.e. $U^{\#} \subseteq \mathbf{2 B}$ and $U \cap F_{3}=1$ ). Then, $d_{i} \geqslant 2, i=1,2$.

We claim that for $j=2$ and $4, p_{j}(U) \geqslant E_{j} / F_{3}$. If false, then for some $j \in\{2,4\}, \quad p_{j}(U)$ and $E_{j} / F_{3}$ generate $F_{j} / F_{3}$. Let $v \in \mathbf{2 A} \cap E_{j}$ satisfy $v F_{3} / F_{3} \notin p_{j}(U)$. Then, $\langle U, v\rangle \leqslant E$ has exactly one element of $2 \mathbf{A}$, hence has complexity 3, contradiction.

Define $U_{j} \leqslant F_{j}$ by $U_{j} / F_{3}=p_{j}(U)$ for $j=2,4 ;\left|U_{j}\right|=16$. We claim that $E \leqslant U_{2} U_{4}$. If not, there is a diagonal element $e^{\prime}=e_{2} e_{4} \in E \backslash U_{2} U_{4}$ with $e_{j} \in F_{j} \backslash U_{j}$ for $j=2,4$ (note that if $e_{j} \in F_{j} \backslash U_{j}$ and $e_{k} \in U_{k}$, then, letting $u \in U$ satisfy $u e_{k} \in F_{3}$, we have $u e^{\prime} \in E_{j} \backslash U_{j}$, contrary to the previous paragraph). Then $V:=\left\langle U, e^{\prime}\right\rangle$ is a 2B-pure eights-group and $\left.p_{j}\right|_{V}$ is a monomorphism. The previous claim, applied to any four-group $U_{0}$ in $V$, implies that $E_{j} / F_{3} \leqslant p_{j}\left(U_{0}\right)$ whence $E_{j}=F_{3}$, for $j=2,4$ and so $E$ is 2B-pure. We then 
finish as in Case 1 by quoting [(3.8), CoGr]. So, the claim holds and we may assume that $E=U_{2} U_{4}$, rank 6 . The claim also implies that there is a 2A-pure four-group $W$ complementing $F_{3}$ in $U_{4}$ and a subgroup $\langle h\rangle \cong \mathbb{Z}_{3}$ in $N(M)$ such that $W=C_{M}(h)$ and $U_{2}=[M, h]$. Thus, $U_{2}$ lies in $L$, the $3 E_{6}(\mathbb{K})$ component of $C(W) \cong \mathbb{T}_{2} E_{6}(\mathbb{K}): 2$. We now quote the complexity result (8.2) to get $U_{2}$ toral in $L$, which implies that $E=W U_{2}$ is toral in $G$.

Subcase 2.2. Subcase 1 does not hold. Then, at least one $d_{j}$ equals 1 . Since diagonal elements exist in $E, d_{j} \geqslant 1$ for both $j$. So, $\left\{d_{2}, d_{4}\right\}=\{1,1\},\{1,2\}$ or $\{1,3\}$. In the first two cases, $E$ may be expanded to a group $U_{2} U_{4}$ as in the last paragraph, and we finish as above. In case $\left\{d_{2}, d_{4}\right\}=\{1,3\}$, we may assume that $d_{2}=1$ and let $U$ be a 2A-pure fours-group in $E_{4}$ (it exists in ker $p_{2}$ since $d_{4}=3$ ). Since $\operatorname{cx}(E) \leqslant 2, r_{4} \leqslant 4$ and so $r_{4}=4$. Also, $r_{2} \leqslant 3$ implies that $r_{2}=3$ and there is $e \in E$ such that $\left\langle e, E_{4}\right\rangle$ has rank 5 and contains three elements of $2 \mathrm{~A}$ (since $d_{4}=3$ and $r_{4}=4$, take $e$ such that $p_{4}(e) \notin E_{4} / F_{3}$ and $\left.p_{2}(e) \neq 1\right)$, a contradiction to $\operatorname{cx}(E) \leqslant 2$.

(9.3) NOTATION. We let $G:=2 E_{7}(\mathbb{K})$, embedded in $H:=E_{8}(\mathbb{K})$ as a central factor of the centralizer of $t \in \mathbf{2 A}$. Let $Z:=\langle t\rangle$.

(9.4) REMARK. For every involution $x \in G \backslash\langle t\rangle,\{x, x t\}$ contains one element each of $2 \mathrm{~B}\left[2 E_{7}\right]$ and $2 \mathrm{C}\left[2 E_{7}\right]$; on the adjoint module for $G, x$ has trace 5 ; their $G$-centralizers have shape $2^{2} D_{6} A_{1}$. There are two classes of elements of order 4 which square to $t, \mathbf{4 A}$ and $\mathbf{4 H}$; their traces on the adjoint module for $G$ are -7 and 25 ; see (2.14).

(9.5) PROPOSITION. (Classification of subgroups of order 8.) We consider groups $E \leqslant G$ of order 8 with $\Phi(E) \leqslant\langle t\rangle \leqslant E$.

(i) Suppose $E \cong 2_{\varepsilon}^{1+2}$. The class distribution for elements of order 4 is of the form $\mathbf{4 A}^{m} \mathbf{4 H}^{n}$, for even integers $m, n ; m+n=2$ or 6 as $\varepsilon=+$ or - . Up to conjugacy, the possibilities are:

\begin{tabular}{|c|c|c|c|}
\hline Distribution & Centralizer & Dimension & Normalizer \\
\hline & None occur. & & \\
\hline $4 A^{6} 4 H^{0}$ & $2 \times \operatorname{PSO}(8, \mathbb{K})$ & 28 & {$[\operatorname{SL}(2,3) \times \operatorname{PSO}(8, \mathbb{K})] \cdot 2$} \\
\hline $4 \mathrm{~A}^{4} \mathbf{4} \mathrm{H}^{2}$ & $2 \times \operatorname{PSp}(8, \mathbb{K})$ & 36 & {$\left[\right.$ Quat $\left._{8} \times \operatorname{PSp}(8, \mathbb{K})\right] \cdot 2$} \\
\hline $\mathbf{4} \mathbf{A}^{2} \mathbf{4} H^{4}$ & Does not occur; & & \\
\hline $\mathbf{4} \mathbf{A}^{0} \mathbf{4} \mathbf{H}^{6}$ & $F_{4}(\mathbb{R} \mathbb{S})$ & 52 & $\operatorname{SL}(2,3) \cdot 2 \times F_{4}(\mathbb{K})$ \\
\hline
\end{tabular}

(ii) Suppose that $E \cong 4 \times 2$. The distribution is $\mathbf{2 A}^{1} \mathbf{2} \mathbf{B}^{\mathbf{1}} \mathbf{2} \mathbf{C}^{1} \mathbf{4 A}^{m} \mathbf{4} \mathbf{H}^{n}, m, n$ even and $m+n=4$. Up to conjugacy, the possibilities are:

$\begin{array}{llll}\text { Distribution } & \text { Centralizer } & \text { Dimension } & \text { Normalizer } \\ \mathbf{2 A}^{\mathbf{1}} \mathbf{2} \mathbf{B}^{1} \mathbf{2 C}^{\mathbf{1}} \mathbf{4 A}^{\mathbf{4}} \mathbf{4 H}^{0} & 4 A_{3} A_{3} \circ \mathbb{T}_{1} & 31 & 4 A_{3} A_{3} \circ T_{1} \cdot 2 \\ \mathbf{2 A}^{1} \mathbf{2} \mathbf{B}^{1} \mathbf{2} \mathbf{C}^{1} \mathbf{4 A}^{\mathbf{2}} \mathbf{4} \mathbf{H}^{2} & 6 A_{5} A_{1} \circ \mathbb{T}_{1} & 39 & 6 A_{5} A_{1} \circ T_{1} \cdot 2 \\ \mathbf{2 A}^{1} \mathbf{2} \mathbf{B}^{1} \mathbf{2} \mathbf{C}^{1} \mathbf{4 A}^{0} \mathbf{4} \mathbf{H}^{4} & \text { Does not occur } & (49) & \end{array}$


(iii) Suppose that $E$ is elementary abelian. Up to conjugacy, there are two cases and their distributions are $\mathbf{2 A}^{1} \mathbf{2} \mathbf{B}^{3} \mathbf{2} \mathbf{C}^{3}$. Under the natural bilinear form (2.16), their radicals have ranks 3 and 1 and the respective centralizers are:

(a) (radical of rank 3) $C(E) \cong \mathrm{SL}(2, \mathbb{K})^{3} \circ \mathrm{Spin}(8, \mathbb{K})$ (amalgamation over a 2B-pure $\left.2^{2}\right), N(E)=C(E) S$, where $S \cong \Sigma_{3}$ permutes the three $\operatorname{SL}(2, \mathbb{K})$ factors and acts as graph automorphisms on the $\operatorname{Spin}(8, \mathbb{K})$-factor.

(b) (radical of rank 1) $C(E) \cong\left[\mathbb{T}_{2} \circ 6 A_{5} A_{1}(\mathbb{K})\right] \cdot 2$ (amalgamation over $\mathbb{Z}_{3}$; if $x \in C(E) \backslash C(E)^{0}, x^{2}=t, x$ inverts the $\mathbb{T}_{2}$-factor and effects a graph automorphism on an $A_{5}-$ factor $), N(E) \cong\left[\mathbb{T}_{2}: \Sigma_{3} \circ 6 A_{5} A_{1}\right]: 2$.

Proof. Use (2.14), (2.18) and orthogonality relations (1.5); see also (9.4); for (ii), note that the nonconjugate involutions of $E$ are ratios of elements of order 4. The last distribution listed in (ii) implies centralizer dimension 49 from the orthogonality relations, though the case does not actually occur. To prove (iii), the rank 1 case, consider the $2 E_{7}$-subgroup of $H$ defined as the centralizer of an $A_{1}$-factor in $\left[\mathbb{T}_{2} \circ 6 A_{5} A_{1}(\mathbb{K})\right]: 2 \leqslant 3 A_{2} E_{6}(\mathbb{K}) \leqslant H$. For the rank 3 case, consider $2^{2} A_{1}^{4} D_{4}(\mathbb{K}) \leqslant 2^{2} D_{4}^{2}(\mathbb{K}): 2 \leqslant H$.

(9.6) COROLLARY. If $\langle t\rangle=Z \leqslant E \leqslant G$ and $E / Z$ is an elementary abelian 2group, it centralizes $y(\bmod Z)$, for some element $y \in \mathbf{4 H}$.

Proof. Without loss, $E / Z$ is maximal elementary abelian in $G$. We assume that (9.6) is false and seek a contradiction. We assume that $E$ normalizes $\mathbb{T}_{(2)}$, by (2.13.iii), hence $E$ normalizes a subgroup of $\mathbb{T}_{(2)}$ whose centralizer has shape $2^{2} D_{6} A_{1}$; see (9.4).

Suppose that $E$ is elementary abelian. If $E$ lies in some $\mathbb{T}_{(2)}$, and the result is clear since $E$ is a subspace of $\mathbb{T}_{(2)}$ and $G \cap \mathbb{T}$ is a maximal torus of $G$. Suppose $E$ is nontoral in $E$. Embed $E$ in $M$, a maximal elementary abelian group in $H \cong E_{8}(\mathbb{K})$. If $M$ has type 1, the above argument, applied to $E \cap \mathbb{T}_{(2)}$, tells us $E \cap \mathbb{T}_{(2)}$ is toral in $G$; since $G$ has no Chevalley involution (2.23), $E=E \cap \mathbb{T}_{(2)}$ and $E$ is toral, contradiction. So, assume $M$ has type 2. We claim that there is a 2A-pure fours-group, $U \leqslant M$, such that $U \cap E=\langle t\rangle$. Then, $E$ does not contain both 2A-components. By (9.4), if $X$ is the 2A-component containing $t$, $X \cap E \leqslant \operatorname{rad}(M)\langle t\rangle$. We take such a $U$ in $X$. The claim tells us that $E \leqslant C_{H}(U) \cong\left[\mathbb{J}_{2} \circ 3 E_{6}(\mathbb{K})\right]: 2$, whose intersection with $G$ has the form $\left[\mathbb{T}_{1} \circ 3 E_{6}(\mathbb{K})\right] \cdot 2$, which is $N_{G}(\langle y\rangle)$ for some $y \in \mathbf{4 H}$.

We may assume that $\exp (E)=4$. Since (9.6) is false, every element of order 4 in $E$ is in $\mathbf{4 A}$. Let $u$ be such an element and set $F:=C_{E}(u)$, of index 1 or 2 in E.

Suppose $E=F$ is abelian. Then, $E$ corresponds to an abelian group in $\operatorname{SL}(8, \mathbb{K}) /\{ \pm 1\} \cong C(u)$, and so is described by (3.1). In particular, maximality implies that $\operatorname{rk}(E) \geqslant 6$. Let $R$ be a rank 6 subgroup of $E$ such that 
$R \cap\langle u\rangle=\langle t\rangle$ and $\exp (R)=2$. We have that every element of order 4 in $\langle R, u\rangle$ is in $R u$. Then, computing fixed points (1.5) of $\langle R, u\rangle$ on the adjoint module, we get $[133+31 \cdot 5+32(-7)] / 64=1$. In the notation of (3.1) (applied to $\langle R, u\rangle$ ), this means that the commuting algebra of the group ' $A$ ' has dimension at most 1 ; thus, $r=3$ and so $\operatorname{rk}(E)=7$. Now, we contradict maximality of $E$ by noticing that there is an outer automorphism of $C(u)$ which centralizes $E$ modulo $\langle t\rangle$.

We have $|E: F|=2$. Let $v \in G$ satisfy $v^{2}=t$ and $u^{t}=u^{-1}$. Then, the possibilities for $Q:=\langle u, v\rangle \cong$ Quat $_{8}$ are discussed in (9.5). Since we ate forbidding $\mathbf{4 H}$, we get that $C(Q) \cong 2 \times \operatorname{PSO}(8, \mathbb{K})$; by $(2.21)$, the maximal elementary abelian groups here have rank 6 or 7 . Also, $E \leqslant C(Q)$, so there is a rank 5 elementary abelian subgroup $R$ of $E$ such that $E$ contains $Q \times R ; R$ is elementary abelian of rank 5. The sum of the traces (1.5) is $133+31 \cdot 5+3 \cdot 32(-7)=-384$, but it should be nonnegative, a contradiction.

(9.7) COROLLARY. Let $Z \leqslant E \leqslant G$. If $E$ is maximal elementary abelian in $G$, it is toral or is nontoral of rank 6 , unique up to conjugacy. If $E / Z$ is a maximal elementary abelian 2-group in $G / Z$, it is nontoral and is conjugate to one of two groups, one of rank 8 and one of rank 7.

Proof. Without loss, $E \leqslant C(y \bmod Z)$, which has shape $\mathbb{T}_{1} \circ 3 E_{6}(\mathbb{K}) .2$ and we also assume that $E / Z$ is maximal in $G / Z$; so, $y \in E$. Suppose $E \leqslant C(y)$; then, $E$ is abelian. We quote (8.2) and use (2.18) to get that each maximal elementary abelian 2-group of $3 E_{6}(\mathbb{K})$ (ranks 5 and 6 , the latter being toral) is centralized by an outer automorphism (one must observe that the nontoral $2^{6}$ in a $\operatorname{PSp}(8, \mathbb{K})$ subgroup of $3 E_{6}(\mathbb{K})$ is toral in the group $\left.3 E_{6}(\mathbb{K})\right)$. So, $E$ is not maximal, a contradiction.

Suppose $E$ is not in $C(y)$. There exists $Q$, nonabelian of order 8 , such that $y \in Q \leqslant E$. Then, by $(2.18), C(Q) \cong F_{4}(\mathbb{K})$ or $\operatorname{PSp}(8, \mathbb{K})$. Any such $Q$ is quaternion; see $(9.5)$.

From (2.21) and (7.3), we have, up to conjugacy, at most three candidates for $E$; they have the form $E=Q \times R$; the ranks of such $R$ are 5,5 and 6 . We shall be done if we show that the two maximal rank 5 groups lead to $E$ which are conjugate in $C(y)$. Let $v_{i}$ be elements of $C(y \bmod \langle t\rangle)$ which induce nonconjugate outer automorphisms of order 2 on $C(y)$. Set $Q_{i}=\left\langle y, v_{i}\right\rangle$, $i=1,2$ and suppoe $C\left(Q_{1}\right) \cong F_{4}(\mathbb{K})$ and $C\left(Q_{2}\right) \cong \operatorname{PSp}(8, \mathbb{K})$. Let $R_{i}$ be a maximal nontoral elementary abelian 2-group of rank 5 in $C\left(Q_{i}\right), i=1,2$. Summing traces over $Q_{1} R_{1}$ we get a contraction in case every element of order 4 in it is in $\mathbf{4 H}$ : $[133+31 \cdot 5+3 \cdot 32 \cdot 25] / 128=21$, whereas $R_{1}$ is selfcentralizing in $F_{4}(\mathbb{K})$. We conclude that there is an element in $Q_{1} R_{1} \cap 4 \mathrm{~A}$, which we may as well take to be $v_{2}$. Thus, $R_{1} \leqslant C\left(Q_{2}\right)$ and it is self- 
centralizing also in $C\left(Q_{2}\right) \cong \operatorname{PSp}(8, \mathbb{K})$, whence $R_{1}$ and $R_{2}$ are conjugate in $C\left(Q_{2}\right)$.

(9.8) THEOREM. (i) $G=2 E_{7}(\mathbb{K})$ has two classes of maximal elementary abelian 2-groups, a toral one of rank 7 and a nontoral one of rank 6 . If $E$ is the nontoral group of rank $6, C_{G}(E) \cong 2^{3} \times \mathrm{SL}(2, \mathbb{K})^{3}, N_{G}(E) / C_{G}(E) \cong 2^{2 \cdot 3}$ : $\left[\Sigma_{3} \times \mathrm{GL}(3,2)\right], \quad C_{H}(E) \cong 2^{3} \times 2^{3} A_{1}(\mathbb{K})^{4}$ and $N_{H}(E) / C_{H}(E) \cong 2^{1 \cdot 2+2 \cdot 3}$ : $\left[\Sigma_{3} \times \mathrm{GL}(3,2)\right]$. The distribution is $A^{32} B^{31}$.

(ii) Let $Z=Z(G)$. Then, $G / Z=E_{7}(\mathbb{K})$ has two classes of maximal elementary abelian groups, $E / Z$, of ranks 8 and 7 ; representative groups $E$ have shapes Quat $_{8} \times 2^{k}$, for $k=6,5$, and so both are nontoral. Say these groups have type 1 and type 2. Each satisfies $C(E)=Z(E)$.

If $E$ has type $1, N_{G}(E) / C_{G}(E) \cong\left[\mathrm{Dih}_{8} \times 2^{6}\right]: \mathrm{Sp}(6,2)$, and $N_{G}(E) / E \cong$ $2^{7}: \mathrm{Sp}(6,2)$ (the elementary abelian $2^{7}$ is an indecomposable module for $\mathrm{Sp}(6,2)$, with ascending factors of dimensions 6 and 1).

If $E$ has type $2, N_{G}(E) / E \cong 2^{2 \cdot 2 \cdot 1+1 \cdot 2 \cdot 3}:\left[\Sigma_{3} \times \Sigma_{3} \times \operatorname{GL}(3,2)\right]$.

Proof. Use (9.5), (9.6) and (9.7) for everything except the statements about the centralizers and normalizers.

(i) Let our nontoral maximal be $E=F \times D$, where $F$ is an eights-group whose involutions are in the $H$-class $2 \mathbf{A}$ and $\operatorname{rk}(D)=3$; we embed $E$ in a type 2 maximal of $H$ and take $D$ as a subgroup of the $2 A$-component which does not contain $F$. Then, $D \leqslant C_{H}(F)^{0} \cong F_{4}(\mathbb{K})$. The class distribution $A^{4} B^{3}$ of $D \leqslant C_{H}(F)^{0}$ implies that $D$ is toral (see(7.3)) and $C_{H}(F)^{0} \cap C(D) \cong 2^{3} A_{1}(\mathbb{K})^{4}$. Recall that relations among the involutions generating the centers of the $\operatorname{SL}(2, \mathbb{K})$ are given by words in the extended Hamming code; see (2.3) and [CoGr, (3.8)]. The statements about the normalizers and the distribution are easy to deduce from the above and (7.3).

(ii) This is more subtle, due to the quaternion subgroups of the two groups $E$, described in (9.7).

Suppose $E$ has type 1 . We take $E \leqslant N(\langle y\rangle)$, as in (9.6). Let $E=Q \times F$, where $Q \cong$ Quat $_{8}$ and $F$ has rank 6 and lies in $C(Q)^{\prime} \cong \operatorname{PSp}(8, \mathbb{R})$. Then, by (8.2), $F$ is toral in $C(y) \cong 3 E_{6}(\mathbb{K})$ and so $E_{y}:=\langle y\rangle \times F$ is toral in $G$. Its centralizer $C_{y}$ is a torus in $G$ and we get $N\left(E_{y}\right) / C_{y} \cong W_{E_{7}} \cong \operatorname{Sp}(6,2) \times 2$. The image of $Q$ in this is the direct factor of order 2 since $Q$ centralizes $N\left(C_{y}\right)^{\prime}$ but $\operatorname{not} C_{y}$.

We claim that $E_{y}$ is the unique abelian maximal subgroup of $E$ which centralizes a maximal torus. Since $E=Q \times F$, where $F$ is elementary abelian, any abelian maximal subgroup of $E$ has the form $\langle u\rangle \times F$, where $u \in Q$ has order 4. Since $C(Z(E)) \cong \mathbb{J}_{7} .2$ (torus extended by -1 from the Weyl group), we observe that $F \times\langle y\rangle$ is the only possibility, proving the claim.

It follows that $N(E) \leqslant N\left(E_{y}\right)$ and the structure of $E_{y}$ given above (with the 
observation that $E_{y} / \mathbb{T}_{(2)}$ is a fixed point for the action of $W_{E_{7}}^{\prime}$ ) leads to $C(E)=Z(E), \quad N(E) / E \cong 2^{7}: \mathrm{Sp}(6,2)$ (socle of dimension 6), $N(E) / C(E) \cong$ $\left[\mathrm{Dih}_{8} \times 2^{6}\right]: W_{E_{6}} \times \mathrm{Dih}_{8}$, as in the statement of the theorem. The indecomposability statement is due to $\left[\langle y\rangle, N\left(E_{y}\right)^{\prime}\right]=\Omega_{1}\left(E_{y}\right)$, which follows from the irreducibility of $N\left(E_{y}\right)^{\prime}$ on $\Omega_{1}\left(E_{y}\right) /\langle z\rangle$ and the structure of $\mathbb{T}$ as a $W_{E_{8}}$-module.

We mention that since $\operatorname{dim}\left(C_{G}(Q)\right)=36$, orthogonality relations (1.5) show that $Q$ has distribution $\mathbf{2 A}^{\mathbf{1}} \mathbf{4} \mathbf{A}^{4} \mathbf{4} \mathbf{H}^{2}$; see (9.5).

Suppose $E$ has type 2 . We have $\operatorname{rk}(Z(E))=6$ and $Z(E)$ contains an elementary abelian 2-group, $F$, which is maximal in $C(Q)^{\prime} \cong F_{4}(\mathbb{K})$; so $E=Q \times F$. Since $C_{H}(F)^{0} \cong 2^{2} D_{4}(\mathbb{K})$ and since a maximal 2-group in $H$ which contains $Z(E)=F Z(Q)$ must be of type 2 (see (2.17)) with $F$ as a 2Acomponent, we see that $\langle z\rangle=Z(Q)$ must be in the other $2 \mathrm{~A}$-component and then that the distributions $\left(\mathbf{A}^{28} \mathbf{B}^{3}\right.$ for $F$ and $\mathbf{A}^{31} \mathbf{B}^{32}$ for $\left.F Z(Q)\right)$ with (2.13.v) give $C_{H}(Z(E)) \cong 2^{3} A_{1}(\mathbb{K})^{4} \times 2^{3}$. Let $S_{i}, i=1,2,3,4$, be the factors of $C_{H}(Z(E))^{0}$, where $S_{1}$ satisfies $C_{H}\left(S_{1}\right)=G$.

Let $U$ be the fours-group in $C(Z(E))^{0}$ which is 2B-pure in $H$. Then $C_{H}(U) \cong 2^{2} D_{4}(\mathbb{K})^{2}: 2$ and $N_{H}(U) \cong 2^{2} D_{4}(\mathbb{K})^{2}:\left[\Sigma_{3} \times 2\right]$; see [CoGr, (3.8)]. Thus, if we consider $Q \times N_{C(Q)^{\prime}}(F) \cong \mathrm{Quat}_{8} \times 2^{5} \cdot 2^{2 \cdot 3} \cdot\left[\mathrm{GL}(3,2) \times \Sigma_{3}\right]$, we see that $Q$ is in the subgroup of $C(Z(E))$ which is the fixed point group of some $S \cong \Sigma_{3}$, which induces a standard group of graph automorphisms on both factors of $C_{H}(U) \cong 2^{2} D_{4}(\mathbb{K})^{2}\left(S\right.$ is a subgroup of $N_{C(Q)}(F) \cap C(Q)$ centralizing $\left.2^{3} \cdot \mathrm{GL}(3,2)\right)$. In fact, $Q \leqslant C(Z(E))^{0}$ since the actions on $F$ of $Q$ and $N_{C(Q)}(F)$ (acting as $\mathrm{GL}(3,2)$ ) commute. Thus, $Q$ lies in a $\mathrm{SL}(2, \mathbb{K})$-subgroup, $K=C_{S_{1} S_{2} S_{3}}(S)$, which is diagonally embedded in $S_{1} \times S_{2} \times S_{3}$. We have $N_{K}(Q) \cong \mathrm{SL}(2,3) \cdot 2$. Consequently, we see that $N(E) \cap N(Q)$ maps onto $\Sigma_{3} \times \Sigma_{3} \times \mathrm{GL}(3,2)$. We have $Q \leqslant C(Z(E))^{0} \cap E=Q U=Q \times U$, whence $|N(E): N(E) \cap N(Q)|$ divides $|U|^{2}=16$. We get equality as follows. The quasiprojections $Q_{i}$ of $Q$ satisfy $Q_{1} Q_{2} Q_{3}=Q_{1} \times Q_{2} \times Q_{3}$ and $C_{Q_{1} Q_{2} Q_{3}}(Q)$ $=Q Z\left(Q_{1} Q_{2} Q_{3}\right)$ and $\left[Q_{1} Q_{2} Q_{3}, Q\right]=Z\left(Q_{1} Q_{2} Q_{3}\right)=U \times Z(Q)$. It follows that $|N(E): N(E) \cap N(Q)|=16$ and that the image of $Q_{1} Q_{2} Q_{3}$ in $N(E) / E C(E)$ is a subgroup of order 16 commuting with $N_{C(Q)^{\prime}}(E)$.

(9.9) REMARK. Here is another description of the two maximal groups for $G:=2 E_{7}(\mathbb{K})$.

Let $f$ be an element of order 4 in $G$ with $Z=Z(G)=\left\langle f^{2}\right\rangle$ and $C(f) \cong 4 A_{7}$; then $C_{G}(f Z)=\{x \in G \mid[f, x] \in Z\}=C(f)\langle y\rangle$, where $y^{2}=f^{2}$ and $y$ induces a graph automorphism on $C(f)$.

Let $\pi: \operatorname{SL}(8, \mathbb{K}) \rightarrow C(f) / Z$ be an epimorphism.

Then, for suitable choices of $y^{\prime} \in C(f) y$, our subgroups are $\left\langle y, F^{\pi}\right\rangle$, where $F$ is a group of shape $2^{1+2 r} \circ 8 \times 2^{s-1}$, for $(r, s)=(1,4)$ and $(3,1)$; the ranks are 
$2 r+s+1=7$ and 8 and the fixed points of $y^{\prime}$ in the respective cases are $2 \times \operatorname{PSO}(8, \mathbb{K})$ and $2 \times \operatorname{PSp}(8, \mathbb{K})$.

\section{TyPes $E_{6}, E_{7}$ AND $E_{8}, p \geqslant 5$}

(10.1) NOTATION. Let $G$ have type $E_{6}, E_{7}$ or $E_{8}$. Since $p \geqslant 5$, we take $Z=1$ without loss of generality.

(10.2) LEMMA. In $E_{6}(\mathbb{K}), E_{7}(\mathbb{K})$, and $E_{8}(\mathbb{K})$, every elementary abelian p-group is toral for $p \geqslant 7$.

Proof. We need consider only prime divisors of the order of the Weyl group. So, $p \leqslant 7$.

If $p=7, G=E_{8}(\mathbb{K})$. Take a maximal elementary abelian $p$-group $E \leqslant N\left(\mathbb{T}_{(7)}\right)$. Then, $E$ centralizes an element $x$ of order 7 such that satisfies: $C(x)$ has factors $A_{6} A_{1} \mathbb{T}_{1}$ (reason: the stabilizer in the Weyl group has index prime to 7 and there is only one such class of cyclic subgroups of order 7 with such factors in the centralizer; see [Table 6, CoGr]). It follows that $E$ lies in a subgroup of the form $\operatorname{SL}(7, \mathbb{K}) \mathbb{T}_{2}$, whence $E$ is toral.

(10.3) LEMMA. Let $p=5$. Then $E$ is toral with the unique (up to conjugacy) exception of $G=E_{8}(\mathbb{K})$ and $\mathrm{rk} E=3, E^{\#} \subseteq 5 \mathrm{C}$. For such $E, C(E)=E$ and $N(E) / E \cong \mathrm{SL}(3,5)$. Furthermore, $N(E)$ splits over $E$.

Proof. Let $G=E_{8}(\mathbb{K})$. The Sylow 5-group $P$ of $W$ has type $5 \times 5$ and we may see one in a natural $\left(W_{A_{4}} \times W_{A_{4}}\right)$-subgroup. Its action on $\mathbb{T}_{(5)}$ has fixed points a 1-dimensional space $\langle z\rangle$ and $C(z)=X_{1} X_{2}$, a nontrivial central product with $X_{i} \cong \operatorname{SL}(5, \mathbb{K})$ for $i=1,2$ and $X_{1} \cap X_{2}=\langle z\rangle$. Then, if $E \leqslant C(z)$ is nontoral in $G$, rk $E \geqslant 3$ and so by (3.1) $z \in E$, rk $E=3$ and each quasiprojection $Q_{i}$ is nonabelian, whence $Q_{i} \cong 5_{\varepsilon}^{1+2}$; since $Q_{i} \leqslant X_{i} \cong$ $\operatorname{SL}(5, \mathbb{K}), \varepsilon=+$. Since $N_{X_{i}}\left(Q_{i}\right)=5^{1+2}: \operatorname{SL}(2,5)$, there is only one possibility for $E$, up to conjugacy. We have $N_{C(z)}(E)=5^{3}:\left[5^{2}: \mathrm{SL}(2,5)\right]$; in particular, all elements of $E \backslash\langle z\rangle$ are in the same conjugacy class. Clearly, $E=C(E)$ and $\operatorname{dim} C(E)=0$, so, using (1.5), $0=\Sigma_{x \in E} \operatorname{tr}(x)$ and $\operatorname{tr}(x)=-4$ for $x \in\langle z\rangle^{\#}$ imply $\operatorname{tr}(x)<0$ for all $x \in E^{\#}$, whence by [Table $\left.6, \mathrm{CoGr}\right], E^{\#}$ consists of conjugates of $z$. We may now consider the embedding of $E$ in $C(x)$, for all $x \in E^{\#}$ to conclude that $N(E) / E \cong \mathrm{SL}(3,5)$. One can see that this extension is split by using Gaschütz's theorem [Hup, I.17.4], since the above discussion implies that $C(z) \cap N(E)$ splits over $E$.

Incidentally, $H^{2}\left(\mathrm{SL}(3,5), \mathbb{F}_{5}^{3}\right) \neq 0[\mathrm{McL}]$, and a nonsplit extension does exist as a maximal 5-local in the Lyons sporadic simple grou [Ly].

Now, let $G=3 E_{6}(\mathbb{K})$. We prove that any $E$ is toral. So, we suppose that $E$ is nontoral and we seek a contradiction. 
We may assume $E$ normalizes $\mathbb{T}_{(5)}$. Thus, $\left|E: E \cap \mathbb{T}_{(5)}\right|=5$ and rk $E \geqslant 3$. Since $W_{E_{6}}$ contains $W_{D_{5}} \cong 2^{4}: \Sigma_{5}$, an element $x \in E \backslash \mathbb{T}(5)$ has Jordan canonical form $J_{5}+J_{1}$. Therefore, $\operatorname{rk}\left(E \cap \mathbb{T}_{(5)}\right)=2$. Since the root system $E_{6}$ contains the root system $A_{5} A_{1}$, Sylow's theorem for the Weyl group implies that $E$ is contained in a subgroup of the form $X_{1} X_{2}$, where $X_{1} \cong \mathrm{SL}(6, \mathbb{K})$, $X_{2} \cong \operatorname{SL}(2, \mathbb{K})$ and $\left|X_{1} \cap X_{2}\right|=2$. Any abelian 5-group in $X_{1} X_{2}$ is toral by (3.1), so no such $E$ exists.

Finally, we take $G=2 E_{7}(\mathbb{K})$. Table 6 in [CoGr] shows one rational class of elements of order 5 (class $5 \mathrm{~J}$ ), having centralizer with factors $A_{1} D_{4} \pi_{2}$. We get $E$ toral by quoting results for type $D$ and observing that each quasisimple factor here has center of order prime to 5 .

\section{1. $E_{6}, E_{7}$ AND $E_{8}, p=3$}

(11.1) NOTATION. Until the end of this section, $p=3 \neq \operatorname{char}(\mathbb{K}), \omega$ is a primitive cube root of unity in $\mathbb{K}$ and $E$ is an elementary abelian 3-group. Until further notice, $G=E_{8}(\mathbb{K})$. Elements of order 3 in $G$ are discussed in

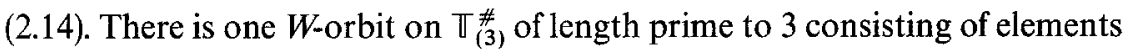
with trace 5 and with centralizers of shape $3 A_{2} E_{6}$. By using this observation for $E_{8}(\mathbb{K})$ and a similar one for $E_{6}(\mathbb{K})$ (see (2.14)), we get that $E$ normalizes $U \cong 3^{2}$ with $C(U) \cong 3^{2} A_{2}^{4} ; U$ is 3B-pure. Let $X_{i}, i=1, \ldots, 4$, be the four central factors of $C(U),\left\langle z_{i}\right\rangle=Z\left(X_{i}\right)$. Also, the structure of $W_{E_{8}}$ implies that $N(U) / C(U) \cong \mathrm{GL}(2,3)$, which acts faithfully on $U$ and induces $\Sigma_{4}$ on the set of four factors (in fact, GL(2,3) is the group of a tetracode (see (2.3)), via its monomial action based on $\left\{z_{i} \mid i=1, \ldots, 4\right\}$. Thus, the relations satisfied by $\left\{z_{i} \mid i=1, \ldots, 4\right\}$ correspond to tetracode words. This is so because the minimum length of a relation is 3 and $\operatorname{rk}(Z(C(U)))=2$; see (2.3).

(11.2) HYPOTHESIS. Until further notice, we assume that $E \leqslant C(U)$. Let $Q_{i} \geqslant\left\langle z_{i}\right\rangle$ be the quasiprojections (see (2.6)) of $E$ with respect to the $X_{i}$. Thus, $E \leqslant Q:=Q_{1} Q_{2} Q_{3} Q_{4}$.

(11.3) LEMMA. Suppose $\langle U, e\rangle \cong 3^{3}$ for some $e \in Q$ of the form $e_{1} \cdots e_{r}$, where $e_{i} \in Q_{i} \backslash U$. The class distribution (see (1.3)) of $\langle U, e\rangle$ is as follows:

$$
\begin{aligned}
& r=1 \quad \mathbf{A}^{12} \mathbf{B}^{8} \mathbf{C}^{0} \mathbf{D}^{6} \quad \text { centralizer: } \quad T_{2} A_{2}^{3} \quad \operatorname{dim} 26 \\
& r=2 \quad \mathbf{A}^{0} \mathbf{B}^{8} \mathbf{C}^{18} \mathbf{D}^{0} \quad \mathbb{T}_{4} \circ\left(3 A_{2}\right)^{2} \operatorname{dim} 20 \\
& r=3 \quad \mathbf{A}^{0} \mathbf{B}^{26} \mathbf{C}^{0} \mathbf{D}^{0} \quad \mathbb{T}_{6}: 3 \circ 3 A_{2} \quad \operatorname{dim} 14 \\
& \boldsymbol{r}=4 \quad \mathbf{A}^{18} \mathbf{B}^{8} \mathbf{C}^{0} \mathbf{D}^{0} \quad \mathbb{\pi}_{8}: 3^{2} \quad \operatorname{dim} 8 .
\end{aligned}
$$


(For $r=1,2,3$, the respective amalgamations for the central products are over $3^{2}, 3^{2}, 3$.)

Proof. For all $r$, the structure of $C(\langle U, e\rangle)$ is easy to get. Here are some hints. If $e_{k}$ is nontrivial, $C_{X_{k}}\left(e_{k}\right)=\mathbb{T}_{2}$ and $C_{X_{k}}\left(e_{k} \bmod U\right)=\mathbb{T}_{2}: 3$. Also, $C:=C(\langle U, e\rangle)$ is connected unless $r \geqslant 3$, the minimum weight of the tetracode. Without loss, we may change notation to make $e_{k}$ nontrivial for $k=1,2,3$ (possibly $e_{4}$ is also nontrivial). For any 3 -set in $\{1,2,3,4\}$, there is a unique 1-space in the tetracode supported by that 3 -set.

As we show below, the distribution follows easily from orthogonality relations (1.5) on $\langle U, e\rangle$ and the observations that if $r=1,\left|x^{C(U)} \cap U x\right|=6$ for all $x \in\langle U, e\rangle \backslash U$ and if $r \geqslant 2, x^{C(U)} \cap U x=\langle U, e\rangle \backslash U$ (which has cardinality 18) for all $x \in\langle U, e\rangle \backslash U$. Details follow.

Let $r=1$. Then $x=e$ is an element of some $X_{i}$ and so is in a fundamental $\mathrm{SL}(2, \mathbb{K})$, whence it is in 3D and $\operatorname{tr}(x)=77$. If $y \in\langle U, e\rangle \backslash\left(\left\langle z_{1}, x\right\rangle \cup U\right)$, we may write $y=z^{\prime} x^{\prime}$, where $z^{\prime} \in U \backslash\left\langle z_{1}\right\rangle$ and $x^{\prime} \in\langle x\rangle^{\#}$. The action of $N(U) \cap N\left(\left\langle x, z_{1}\right\rangle\right)$ is transitive on the set of 12 such $y$. It follows that $26=27^{-1}\{248+8 \cdot 5+6 \cdot 77+12 \operatorname{tr}(y)\}=27^{-1}\{750+12 \operatorname{tr}(y)\}$, whence $\operatorname{tr}(y)=-4$, i.e. $y \in \mathbf{3 A}$.

Now, let $r \geqslant 2$. Then $N(U) \cap N(\langle U, e\rangle)$ is transitive on $\langle U, e\rangle \backslash U$. Let $y \in\langle U, e\rangle \backslash U$ and set $q:=248+8 \cdot 5+18 \operatorname{tr}(y)=288+18 \operatorname{tr}(y)$. For $r=2,3$ and $4, q=20 \cdot 27=540,14 \cdot 27=378$ and $8 \cdot 27=216$, which lead to $\operatorname{tr}(y)=14,5$ and -4 , respectively, i.e. to respective classes $\mathbf{3 C}, \mathbf{3 B}$ and $\mathbf{3 A}$.

(11.4) DEFINITION. Let $E$ be an elementary abelian group of order 9 in $G=E_{8}(\mathbb{K})$ which is 3B-pure. We say $E$ is of c-type $A_{2}^{4}$, c-type $T_{4} D_{4}$ respectively, iff $C(E)$ has factors of these forms. Up to conjugacy, these are the only two possibilities; a 3B-centralizer has.form $3 A_{2} E_{6}$ and so orthogonality relations (1.5) and knowledge of $A_{2}$ and $E_{6}$ (2.14) give two possibilities for $C(E)$, namely $C(E) \cong \mathbb{T}_{4} D_{4}: 3$ and $3^{2} A_{2}^{4}$ (for c-type $T_{4} D_{4}$, an element of order 3 outside $C(E)^{0}$ acts with eigenvalues $\left(\omega^{2}, \omega^{-2}\right)$ on the Lie algebra of the torus and has Jordan canonical form $J_{2}+J_{2}$ on $\mathbb{T}_{4(3)} \cong 3^{4}$; it effects an outer automorphism on the $D_{4}$-factor; this follows from $(11.3, r=3)$ ) and the embedding of $2^{2} D_{4}(\mathbb{K}): \Sigma_{3}$ in $F_{4}(\mathbb{K})$.

(11.5) LEMMA. Assume the notation of (11.3). Let $E$ be a maximal nontoral elementary abelian 3-group such that $C(E) \cap \mathbf{3 A} \neq \varnothing$ (we do not make an assumption about an embedding of $E$ in $N(U)$ ). Then rk $E=5$ and there are two G-conjugacy classes of such groups, described here as subgroups of $C(x) \cong \mathrm{SL}(9, \mathbb{K}) /\langle\omega I\rangle($ see $(3.1))$, for $x \in \mathbf{3 A}$ :

TYPE 1: the image modulo $\langle\omega I\rangle$ of $a 3^{1+2} \circ 9 \times 3 \times 3$ subgroup; 
$C(E) \cong 3^{3} \times \mathbb{T}_{2}$ and $N(E) / C(E) \cong 3^{3}: \operatorname{SL}(3,3) \times \Sigma_{3} ;$ there is a subgroup $E_{0}$ of index 3 such that $E_{0}=(E \cap 3 \mathrm{~B}) \cup\{1\}$; the orbits of $N(E)$ on $E \backslash E_{0}$ have lengths 6 (class 3D) and 156 (class 3A); also, there is a subgroup $E_{00}$ of order 3 in $E_{0}$ which is normal in $N(E)$, namely $E_{00}=E_{0} \cap \mathbb{T}_{2}$. The class distribution for $E$ is $\mathbf{3 A}^{156} \mathbf{B}^{80} \mathbf{D}^{6}$ and a rank 2 subgroup of $E_{0}$ has c-type $A_{2}^{4}$ iff it contains $E_{00}$ (otherwise c-type $T_{4} D_{4}$ ); see (11.4); the group $E_{0}$ is nontoral.

TYPE 2: the image modulo $\langle\omega I\rangle$ of a $3^{1+4} \circ 9$-subgroup; $C(E)=E$. Also, $N(E) \cong 3^{5}: 3^{4}:[\operatorname{Sp}(4,3) \circ 4]$ and $E_{0}:=(E \cap 3 \mathbf{B}) \cup\{1\}$ is a toral subgroup of index 3 , set-theoretically complemented by $E \cap 3 \mathrm{~A} ;$ we have $C\left(E_{0}\right) \cong \mathbb{T}: 3$ and $\left.N\left(E_{0}\right)=\mathbb{T} .[\mathrm{Sp}(4,3) \times 3) .2\right]$. The class distribution is $\mathbf{3 A}^{162} \mathbf{B}^{80}$. Every rank 2 subgroup of $E_{0}$ has c-type $T_{4} D_{4}$.

(11.6) NOTATION. In each case above, the group $E_{0}$ is called the 3Bradical of $E$ and for type $1, E_{00}$ is called the $\mathbf{3 B}$-socle of $E$. We write $E_{0}=\operatorname{rad}(E)$ and $E_{00}=\operatorname{soc}(E)$. In case $E$ has type 2, we call $E_{0}$ by both of the names $\operatorname{rad}(E)$ and $\operatorname{soc}(E)$.

Proof of $(11.5)$. Since $C(x) \cong \mathrm{SL}(9, \mathbb{K}) /\langle\omega I\rangle$, we consult (3.1) to see that a nontoral elementary abelian 3-subgroup of $C(x)$ is conjugate to an image modulo $\langle\omega I\rangle$ of a nonabelian subgroup of $\operatorname{SL}(9, \mathbb{K})$. The statement about the normalizer for type 2 is easily deduced from the structures of $C(x)$ and $T . W_{E_{8}}$. For type 1, we requre a somewhat detailed discussion and we refer ahead to the result (11.13) on $3 E_{6}(\mathbb{K})$, which is independent of (11.5). The connection is as follows. Let $x \in 3 \mathrm{~B}$; then $C(x) \cong 3 E_{6} A_{2}(\mathbb{K})$ and the $3 E_{6}$-factor $X_{1}$ contains a nontoral group $E_{1}$ of rank 4 such that $N_{X_{1}}\left(E_{1}\right) \cong 3^{1+3+3}: \operatorname{SL}(3,3)$ and the $3 A_{2}$-factor $X_{2}$ contains a group $E_{2} \cong 3 \times 3$. Thus, $E_{1} E_{2}$ has rank 5 and $(11.3 . r=1)$ implies that it contains an element of $3 \mathbf{A}$, so is conjugate to the type 1 group mentioned above. Since $\operatorname{SL}(3,3)$ is not involved in $W_{E_{8}}, E_{1} E_{2}$ is nontoral. Since $C(E)^{0}=C_{X_{2}}\left(E_{2}\right) \cong \mathbb{T}_{2}$ and $C\left(C(E)^{0}\right)^{\prime}=X_{1}$, we conclude that $N(E) \leqslant N(\langle x\rangle)$ and the rest is easy.

(11.7) LEMMA. Assume (11.2). Then E centralizes an element of $3 \mathbf{A}$.

Proof. If $E \leqslant U$, this is obvious from (11.3). So, assume $E$ contains some $\langle U, e\rangle$ as in (11.3). Suppose that such an $e$ has $r \leqslant 2$. We are done if $r=1$, so we assume $r=2$. Then the shape of $C(\langle U, e\rangle$ ) (given in $(11.3 . r=2)$ ) shows that $E$ is toral since all the quasiprojections are abelian (see (3.1)), whence the result. Thus, we may assume that all such $e$ have $r \geqslant 3$. It follows that the projection of $E / U$ to $Q_{1} Q_{2} / U$ is a monomorphism. Let ( $a b c d$ ) denote the coordinates modulo $U$ of an element of $Q$ with respect to the decomposition of $Q / U$ into the product of $Q_{i} U / U$. We use additive notation for $Q / U$. By (11.3), we may assume that $E$ contains no element with $r=4$. It follows that 
every element of $E \backslash U$ has $r=3$. We may assume that $E$ is nontoral, whence rk $E \geqslant 4$. Thus, $E$ contains two such elements, say $x$ and $y$, with $r=3$ which are independent modulo $U$.

CASE 1. There are $x$ and $y$ with the zero for $x$ at index 1 and the zero for $y$ at index 2 (we reindex, if necessary). Let $x$ and $y$ be any such elements. Then, $[x, y]=1$ requires $x=(0 a b c)$ and $y=(d 0 e f)$ where each of $\{b, e\}$ and $\{c, f\}$ are dependent $\bmod U$. Since neither $x y$ nor $x y^{-1}$ has $r=2$, we conclude that for all $w \in\langle x, y\rangle^{\#},\{v \in\langle x, y\rangle \mid v$ and $w$ have 0 -coordinate at the same index $\}$ is just $\left\{w^{ \pm 1}\right\}$. Even though $C(\langle U, x\rangle)$ is disconnected, $C(\langle U, x, y\rangle) \cong \mathbb{T}_{8}$ is the unique torus containing it, whence $E$ is toral, a contradiction.

CASE 2. There are no such pairs. Then, reindexing if necessary, we may assume that every element of $E$ has trivial coordinate at index 1 . Then $E$ centralizes every element with coordinates $(a 000)(\bmod U)$ hence some element of 3A; see $(11.3 . r=1)$.

(11.8) HYPOTHESIS. We drop (11.2) and assume that $E$ is not in $C(U)$. If $e \in E \backslash U,\langle U, e\rangle \cong 3_{+}^{1+2}$.

(11.9) LEMMA. Assume (11.8). Let $e \in E \backslash C(U)$. Suppose that e permutes the $X_{i}$ as the 3-cycle (123)(4); see (11.1). Then $C(\langle U, e\rangle) \cong \operatorname{PSL}(3, \mathbb{K}) \times \operatorname{SL}(3, \mathbb{K})$ or $\operatorname{PSL}(3, \mathbb{K}) \times \mathbb{T}_{2}$, according to whether $e$ is trivial or nontrivial on $X_{4}$. Thus, $E$ is contained in $A \times B \times\langle e\rangle$, where $A \leqslant X_{1} X_{2} X_{3}, A \cong 3^{2}$ (there are two classes of such $A$ in $\operatorname{PSL}(3, \mathbb{K})$; one toral, one not) and $B \cong 3^{2}$ is toral in $X_{4}$. In particular, $C(E) \cap \mathbf{3 A} \neq \varnothing$.

Proof. The first statement is clear from the structure of $N(U)$ sketched in (11.1). The second statement is straightforward and the last follows from (11.3), the case $r=1$, since $e$ centralizes an element of order 3 in $X_{4} \backslash\left\langle z_{4}\right\rangle$.

(11.10) COROLLARY. $E_{8}(\mathbb{K})$ has two conjugacy classes of maximal nontoral elementary abelian 3-subgroups, as in Table II, Section 1 and in (11.5).

Proof. (11.1) through (11.9). Note that types 1 and 2 are not conjugate since the class distributions differ.

(11.11) LEMMA. Let $e \in N(U)$ be an element of order 3 which acts nontrivially on $U$ and centralizes one of the $X_{i}$.

(i) The class distribution of $P:=\langle U, e\rangle$ is $\mathbf{A}^{0} \mathbf{B}^{20} \mathbf{C}^{6} \mathbf{D}^{0}$. Also, $C(P) \cong \operatorname{PSL}(3, \mathbb{K}) \times \operatorname{SL}(3, \mathbb{K})$.

(ii) $A$ 3B-pure subgroup of order 9 in $P$ is of c-type $A_{2}^{4}$.

Proof. (i) (11.9) gives the shape of $C(P)$. Without loss, $i=4$. Let $\langle z\rangle:=C_{U}(e)$. Replacing $e$ by an element of $e U$ if necessary, we observe that 
conjugacy in $N(U)$ gives six conjugates of $e$ and 12 of ex in $P \backslash Z$, for $x \in U \backslash\langle z\rangle$. We have that $\operatorname{dim}\left(C_{G}(P)\right)=8+8=16$. So, using (1.5), $432=27 \cdot 16=248+8 \cdot 5+6 \operatorname{tr}(e)+12 \operatorname{tr}(e x), \quad$ or $\quad 144=6 \operatorname{tr}(e)+12 \operatorname{tr}(e x), \quad$ or $24=\operatorname{tr}(e)+2 \operatorname{tr}(e x)$. Available values for traces here are $-4,5,14$ and 77 . The possible solutions here are $(\operatorname{tr}(e), \operatorname{tr}(e x))=(14,5)$ and $(-4,14)$. We claim that the latter pair is impossible. So, assume $\operatorname{tr}(e)=-4$ and $\operatorname{tr}(e x)=14$. The group $Q:=\langle z, e\rangle$ has class distribution $\mathbf{A}^{6} \mathbf{B}^{2}$ and so centralizer in $E_{8}(\mathbb{K})$ of dimension $9^{-1}\{248-24+10\}=26$, whence the dimension of the $3 E_{6}(\mathbb{K})$ centralizer is $26-8=18$; however, the centralizer dimension for an element of order 3 in $3 E_{6}(\mathbb{K})$ is at least 24 , a contradiction.

Let $\langle z, e\rangle$ be a 3B-pure group of order 9 . We may arrange notation so that the action of $e$ cycles the three components $X_{2}, X_{3}$ and $X_{4}$ and satisfies $z_{i}^{e}=z_{i+1}$ for $i=2,3$ and $4\left(z_{5}:=z_{2}\right)$. Thus, $C(\langle U, e\rangle)$ contains a copy of $\operatorname{PSL}(3, \mathbb{K}) \times \operatorname{SL}(3, \mathbb{K})$, as required. So, (i) follows, and to prove (ii) notice that its denial leads to $\langle e, z\rangle$ of type $\mathbb{T}_{4} D_{4}$ which has no subgroup of the form $\operatorname{PSL}(3, \mathbb{K}) \times \operatorname{SL}(3, \mathbb{K})$.

(11.12) NOTATION. We drop the notations (11.1) and (11.8) and now take $G=3 E_{6}(\mathbb{K})$ and $H=E_{8}(\mathbb{K})$. Recall that $Z(G)=\langle z\rangle$ has order 3 . Then, let $E$ be an elementary abelian group which centralizes $x(\bmod \langle z\rangle), x \in G$ of order 3 with centralizer $C(x)=3^{2} A_{2}^{3}$. If $z_{i}, i=1,2,3$, generate the centers of the respective components $X_{i}$, we may arrange $z_{1} z_{2} z_{3}=1$ by replacing some $z_{i}$ by their inverses if necessary. All relations among the $z_{i}$ follow from this one. Let $U:=Z(C(x)) \cong 3^{2}$ and let $X_{4}:=C_{H}(G) \cong \mathrm{SL}(3, \mathbb{K}) ; U=\langle x, z\rangle$. Distributions will refer to $H$-classes.

(11.13) THEOREM. (i) For $G=3 E_{6}(\mathbb{K})$ and $p=3$, there are (up to conjugacy) one maximal nontoral, of rank 4 , with normalizer $3^{1+3+3}: \operatorname{SL}(3,3)$, and one nonmaximal one, of rank 3 , with normalizer $3 \times 3^{3}: \operatorname{SL}(3,3)$; the nonmaximal one lies in a natural $F_{4}(\mathbb{K})$-subgroup. If $E$ is a rank 4 nontoral, $N\left(O_{3}(N(E))\right)$ has shape $3^{1+3+3}: \mathrm{SL}(3,3)$ (this proves the statement in $(11.5)$ about $N_{E_{8}(C)}(E)$ for $E$ of type 1) and $O_{3}\left(N_{G}(E)\right)$ has $E_{8}$-distribution $\mathbf{B}^{1484} \mathbf{C}^{702}$. (ii) There are nontoral groups in $G$ which normalize $U$ (see (11.12)) but do not centralize $U$; the radical of a type 1 group in $E_{8}(\mathbb{K})$ occurs this way (but not the radical of a type 2 group).

Proof. Let $E$ be a nontoral elementary abelian 3-group.

CASE 1. Suppose $E$ does not centralize $x$. Then $F:=E \cap C(U)$ satisfies $F^{\#} \subseteq 3 \mathrm{~B}$ by $(11.3 . r=3), F$ has index 3 in $E$ and $e \in E \backslash F$ cycles the three factors of $C(x)$ transitively and $C(e) \cap C(x(\bmod \langle z\rangle))$ has the form $\langle z\rangle \times L \times\langle e\rangle$, where $L \cong \operatorname{PSL}(3, \mathbb{K})$. Thus, if $E$ is a maximal such group in $N(U)$, it has rank 4 ; the structure of $L$ implies that there are two possibilities, up to conjugacy. 
We now study $E_{8}(\mathbb{K})$-class distributions and see how such an $E$ may be toral; (ii) will follow. From (11.10) and (11.5), we see that if such an $E$ contains an element of $\mathbf{3 C}$, it is toral. If $E$ is nontoral, there is $e \in E \cap \mathbf{3 B}$, by (11.11). On the other hand, every element of order 3 in $C_{G}(U) e / U$ is conjugate by $C_{G}(U) / U$, so we may use (11.11) and replace $E$ by $F\langle e\rangle$ where $e \in U E \backslash U F$ is in 3B, we see from (11.5) and the facts that $E$ centralizes $X_{4}$ and $\operatorname{dim}(C(E))=8$ that $E$ is nontoral, as required to prove (ii). All elements of $E \backslash F$ are conjugate in $N(E)$ because this is so modulo $U$ and all elements of $e\langle z\rangle$ are conjugate by $U$, so all are in 3B. Thus, $E$ is $\mathbf{3 B}$-pure and so is the radical of a type 1 maximal nontoral (the radical of a type 2 group is toral in $E_{8}(\mathbb{K})$ ).

CASE 2. Suppose that $E$ does centralize $x$ and is maximal nontoral; thus $U \leqslant E$. Let $Q_{i} \geqslant\left\langle z_{i}\right\rangle$ be the quasiprojections of $E$ (see (2.6)) and set $Q:=Q_{1} Q_{2} Q_{3}$. Then $Q_{i}$ is elementary abelian or extraspecial. Suppose one $Q_{i}$ is abelian, say for $i=1$. Then $Q_{1}$ is toral, and since $X_{2} X_{3}=X_{2} \times X_{3}$, the fact that all relations among the $z_{i}$ derive from $z_{1} z_{2} z_{3}=1$ implies that all $Q_{i}$ are abelian, whence $E$ is toral. Therefore, each $Q_{i}$ is extraspecial; $\exp \left(Q_{i}\right)=3$ since $Q_{i}$ is contained in $\operatorname{SL}(3, \mathbb{K})$. Consequently, $U \leqslant Q$.

We now study elements of $E \backslash U$. For $e \in E$, write $e=e_{1} e_{2} e_{3}$ with $e_{i} \in Q_{i}$; these are uniquely determined modulo $U$. We claim that if $e \notin U$, each $e_{i} \notin U$. Let $e$ be a counterexample to this claim. Since $E$ is nontoral, $E \neq\langle U, e\rangle$. Let $f \in E$ satisfy: $e$ and $f$ are independent modulo $U$. Then $e f=f e$ and $r=3$ for every element of $\langle e, f\rangle \backslash U$, whence $e_{i}$ and $f_{i}$ are independent for $i=1,2,3$. Now use the fact that $[e, f]=\Pi\left[e_{i}, f_{i}\right]$ and the fact that relations on the $z_{i}$ follow from $z_{1} z_{2} z_{3}=1$ to contradict independence. The claim follows. So, each $e \in E \backslash U$ is described by $(11.3 . r=3)$. Orthogonality relations (1.3) and the fact that any group of order $3^{3}$ in $C(U)$ with $r=3$ for every noncentral element is nontoral since its centralizer in $E_{8}(\mathbb{K})$ is of dimension 8 and contains $X_{4}$. Such $E$ do exist: take $E=\langle U, e, f\rangle$, where $\left\langle e_{i}, f_{i}\right\rangle=Q_{i}$ for all $i$. Furthermore, the structure of $N_{G}\left(Q_{1} Q_{2} Q_{3}\right)$ show that all such $E$ are conjugate.

Let $\gamma$ be the graph automorphism of $G$ of order 2 whose fixed point subgroup is $K \cong F_{4}(\mathbb{K})$. Then $\gamma$ centralizes a nontoral $E^{*} \cong 3^{3}$, which we may assume is in $N(U)$. If $E^{*}$ does not centralize $U$, it must lie in the radical of $E^{* *}$, a type 1 maximal for $E_{8}(\mathbb{K})$ and so is unique up to conjugacy in $N\left(E^{* *}\right)$; we quote Case 1 . So, we may assume that $E^{*} \leqslant C(U)$; nontorality implies that $\left|E^{*} \cap U\right|=3$ and, by remarks at the end of the last paragraph, $E^{*} U$ is conjugate to $E$. It follows that $E$ and $E^{*}$ are conjugate.

We now look at normalizers. We apply (7.4) to get a subgroup $L \cong 3^{3}: \operatorname{SL}(3,3)$ of $N(E)$. It is clear that from $C(E) \leqslant C(U)$ that $C(E)=\langle z, E\rangle$ and that $C(U) \cap C(E /\langle z\rangle)$ has order $3^{6}$ and commutator subgroup $U$. Let 
$D:=C(E /\langle z\rangle)$; using $N(U)$, we see that $|D|=3^{7}$. Since $C(U) \cap C(E /\langle z\rangle)$ is a maximal subgroup of $D$ which admits $L, D$ has class 3 and the lower central series is a chief series for the action of $\operatorname{SL}(3,3)$ via $L$. So, $N(E) \cong 3^{1+3+3}: \operatorname{SL}(3,3)$, with $D / E$ the module dual to $E /\langle z\rangle$ for $N(E) / D \cong \mathrm{SL}(3,3)$. If we extend $G$ by the graph automorphism, $N(D)$ picks up an involution $\gamma$ such that $F:=C_{D}(\gamma)$ complements $\langle z\rangle$ in $D^{\prime}=E$ and $C_{N(F)}(\gamma)=3^{3}: \operatorname{SL}(3,3)$. The distribution follows from (11.11.i), (11.3. $\left.r=3\right)$ and the transitive action of $N(E)$ on the nonidentity elements of the rank 3 chief factors of $O_{3}(N(E))$.

(11.14) THEOREM. Assume (11.12), except let $Z:=Z(G) \leqslant E \leqslant G$ be such that $E / Z$ is a maximal elementary abelian 3-group. One of the following holds:

(i) $E$ is toral, hence has shape $9 \times 3^{5}$;

(ii) $E$ is abelian, but nontoral, whence $E$ is elementary abelian and is described in (11.13);

(iii) $E$ is nonabelian and has the form $3_{+}^{1+2} \times 3 \times 3$; there are two conjugacy classes of such; groups in one class contain conjugates of $U$, some central and some noncentral; groups from the other class contain conjugates of $U$ and all such lie in the center.

The $E_{8}(\mathbb{K})$-class distributions are $\mathbf{B}^{188} \mathbf{C}^{54}$ and $\mathbf{A}^{12} \mathbf{B}^{152} \mathbf{C}^{72} \mathbf{D}^{6}$. Accordingly, we call the groups type $B C$ and type $A B C D$, respectively. Their centralizers have the shapes $Z(E) \cong 3^{3}$ and $\mathbb{T}_{2} \times 3$ while $N(E) / E C(E) \cong\left[3^{2} \times 3^{2}\right]$ : $[3 \times \mathrm{GL}(2,3)]$ and $\mathrm{Dih}_{12} \times 3^{2}: \mathrm{SL}(2,3)$, respectively (in the latter case, $\mathrm{Dih}_{12}$ corresponds to a group of automorphisms on the toral factor of $C(E)$ ). (If we go up to $G: 2$, these sections expand to $\left[3^{2} \times 3^{2}\right]:\left[\Sigma_{3} \times \mathrm{GL}(2,3)\right]$ and $\operatorname{Dih}_{12} \times 3^{2}: \mathrm{GL}(2,3)$.)

Proof. If $E$ is elementary abelian, we use (11.13) and if $E$ is abelian of exponent 9, we argue as follows that $E$ is toral: The tables in (2.14) or [CoGr] do not list elements of order 9 , though one can get elements whose cube generates $Z(G)$ using [MoPa]. A more elementary argument is the following. We classify automorphisms of order 3 in $3 E_{6}(\mathbb{K})$ by, say, (8.6) of [Kac]; the fixed point subgroups have types $A_{2}^{3}, T_{2} D_{4}, T_{1} A_{1} A_{4}, T_{1} D_{5}$ and $T_{1} A_{5}$; if $y \in E$ has order $9, C(y)$ cannot be of type $A_{2}^{3}$, and in all other cases, $E$ must be a toral subgroup of $C(y)$.

We may assume that $E$ is nonabelian. We may take $E$ to be in the normalizer of a torus, and even to normalize a $G$-conjugate of $U$, whence $U \leqslant E$ by maximality. So, we look in $N(U)$ and use to get the possibilities, which we discuss below. Let $H:=E_{8}(\mathbb{K})$.

CASE 1. $U$ is not central in $E$. From (11.11), we get that 
$C(E) \cap N(U)=Z(E) X_{4} \cong 3^{2} \times \operatorname{SL}(3, \mathbb{K})$. The center is a 3B-pure group of order 27 containing rank 2 groups of both c-types. From (11.3) and (11.11), we get the distribution. Since $U$ is conjugate to a subgroup of c-type $A_{2}^{4}$, such an $E$ occurs also in Case 2, where we analyze its normalizer.

CASE 2. $U$ is central. Then, $U \leqslant E \leqslant X_{1} X_{2} X_{3}$. Let $Q_{i}, i=1,2,3$, be the quasiprojections of $E$ and let $Q:=Q_{1} Q_{2} Q_{3}$. Let $x, y \in E$ such that $[x, y]=z=z_{4}$. Since nonzero tetracode words have weight 3 , it follows that, in the notation of $(11.3), r \geqslant 2$ for all noncentral elements in $\langle x, y\rangle$.

Let $s$ be the minimum value of $r$ for elements of $E \backslash U$. We claim that $s=1$ or 2. If $s=3$, the fact that no tetracode word has length 4 means that for at least one $i, x_{i}$ and $y_{i}$ are dependent modulo $U$ and that we get an element of $\langle x, y\rangle \backslash U$ with $r \leqslant 2$. The claim follows.

Subcase 2.1. $s=1$. For some index $i, Q_{i} \cap E$ has order 9 ; say $i=1$. Since $E^{\prime} \neq 1, E=\left(E \cap Q_{1}\right) \times R$, where $R$ is diagonally embedded in $Q_{2} \times Q_{3}$. All groups of this description (with $E^{\prime}=\langle z\rangle$ ) are conjugate in $N(U)$. Also, $N(E) \leqslant N(U)$ since $U$ is the unique group of c-type $A_{2}^{4}$ in $Z(E)$. We have $C(E) E=\mathbb{T}_{2} \times 3^{1+2}$ and $N(E) / E C(E) \cong\left[\Sigma_{3} \times 3^{2}: \mathrm{SL}(2,3)\right]$. Expanding our picture to $G: 2$, we have $N_{G: 2}(E)=N(E)\langle g\rangle$, where $g$ is an involution normalizing each $X_{i}$ and inducing a standard graph automorphism on each. The fact that $3^{1+2}: \operatorname{SL}(2,3)$ is not embeddable in $\operatorname{SO}(3, \mathbb{K})$ implies that $g$ extends our $3^{2}: \operatorname{SL}(2,3)$-factor to an $\operatorname{AGL}(2,3)$.

Subcase 2.2. $s=2$. Let $v$ be an element of $E \backslash U$ with $r=2$. Let $v=v_{1} v_{2} v_{3}$, with $v_{i} \in X_{i}, i=1,2,3$ (and use similar notations for other elements of $E$ ). If $v$ were in $Z(E)$, every $x=x_{1} x_{2} x_{3}$ would satisfy: $x_{i}$ and $v_{i}$ are dependent modulo $U$, for all $i$ (because the minimum weight in the tetracode is 4 ). Consequently, every quasiprojection of $E$ would be abelian, except possibly at index $i$ where $v_{i} \in U$; since $\langle z\rangle \cap Q_{i}^{\prime}=1, E$ is abelian, a contradiction. So, such a $v$ is noncentral. If $x \in E$ and $[x, v] \neq 1, x_{i}$ and $v_{i}$ are independent modulo $U$ at those two indices where $v_{i}$ is nontrivial. Therefore, $C_{E}(v)$ is abelian of index 3 and it contains no element with $r=2$ outside $U\langle v\rangle$. If $y \in E \backslash U$ is central, $r=3$ and for all $i, y_{i}$ and $v_{i}$ are dependent modulo $U$. Thus, $Z(E)=\langle U, y\rangle$ and $E=\langle U, y, v, x\rangle$, where $x$ must satisfy: $x_{i}$ and $y_{i}$ are independent modulo $U$, for all $i$. Conversely, such an $E$ obviously exists in $C(U)$ and cannot be expanded to a larger group in $N(U)$. Clearly, $C(E)=Z(E)$. Also, $C(Z(E))$ is in $C(U)$ and has the form $\mathbb{T}_{6}: 3$. The explicit forms of the elements $v, x$ and $y$ and the fact that $3 \mathrm{C} \cap E=U v \cup U v^{-1}$ means that $N_{C(Z(E))}(E)$ induces $3^{3}$ on $E$ (via conjugation with cube roots of the $y_{i}$ ). Note also that the distribution here is $\mathbf{B}^{188} \mathbf{C}^{54}$. Thus, the group of Case 1 is conjugate to this group $E$.

What about $N(E)$ ? Since $\langle z\rangle$ is normalized by $N(E), N(E)$ induces a subgroup of $\operatorname{AGL}(2,3)$ on $Z(E) \cong 3^{3}$. To get the normalizer, we let 
$F:=\langle U, x, y\rangle$, a 3B-pure group of order 81 which is the radical of a type 1 maximal elementary abelian in $G$; see (11.13); there are three such groups $F$. Recall, that $N:=N(F) \cong 3^{1+3+3}: \operatorname{SL}(3,3)$. Clearly, $E \leqslant R:=O_{3}(N(F))(E$ looks like $\left.3^{1+3+1}\right)$ and we get $N_{N}(E) / E \cong\left[3^{2} \times 3^{2}\right]: \operatorname{GL}(2,3)$, with each $3^{2}$ factor representing an irreducible and faithful GL(2,3)-module. Notice that $N_{N}(E)$ normalizes each of the four subgroups between $Z(E)$ and $E$ (since there is exactly one containing elements of $3 \mathrm{C}$, the image of $N_{N}(E)$ in this $\Sigma_{4}$ is at most $\mathbb{Z}_{2}$; however, the actions of $N_{N}(E)$ on both $F / Z(E)$ and $E / F$ are equivalent since these factors are in duality). Using the previous paragraph, we have the required structure of $N(E)$.

Going up to $G: 2$ (extension of $G$ by graph automorphism, we get $\left[3^{2} \times 3^{2}\right]:\left[\Sigma_{3} \times \mathrm{GL}(2,3)\right]$, since the fixed points of an involutory outer automorphism of $G$ may be arranged to centralize exactly a $3^{3}: \operatorname{SL}(3,3)$ subgroup of $N(F)$. Note that a $\Sigma_{3}$-subgroup which commutes with a GL(2,3)subgroup acts as a subgroup of the group of units of the commuting algebra on the direct sum $\left[3^{2} \times 3^{2}\right]$ of irreducible GL(2,3)-modules, because its action on this module corresponds to its action on the three $G$-conjugates of $F$ in $E$.

(11.15) NOTATION. $G=2 E_{7}(\mathrm{~K})$.

(11.16) THEOREM. An elementary abelian 3-subgroup is toral, with the following conjugacy classes of exceptions (the rank 4 group is maximal elementary and the rank 3 group is not).

rk $E=3,4$;

$C(E) \cong 3^{3} \times \mathbb{T}_{1}$ in either case;

$N(E) \cong\left[3^{3}: \operatorname{SL}(3,3) \times \mathbb{T}_{1}\right] \cdot 2,\left[3^{1+3+3}: \operatorname{SL}(3,3) \circ \mathbb{T}_{1}\right] \cdot 2$, in the respective cases (note the nonsplitting). Both normalizers map onto $\operatorname{AGL}(3,3)$ and $\mathrm{O}(2, \mathbb{K})$.

Proof. We have $E \leqslant C(x)$, where $x$ has order 3 and $C(x) \cong 3 E_{6} \circ \mathbb{T}_{1}$ (group of order 3 amalgamated). Let $E_{1}$ and $E_{2}$ be the quasiprojections of $E$; both $E_{i}$ are abelian. Note that $\exp E_{1}$ divides 3 iff $\exp E_{2}$ does.

Suppose $\exp E_{1}=3$. Then, $E_{2} \leqslant Z(C(x))$ and so $E=E_{1}$. The discussion for $3 E_{6}(\mathbb{K})$ applies here to give just two conjugacy classes of nontoral $E$, of ranks 3 and 4.

Suppose $\exp E_{1}=9$. Then $E_{1}$ lies in $C(y)$, where $y^{3}=x$ and $y \in L$, the $3 E_{6}(\mathbb{K})$-component of $C(x)$. We get possible shapes of $C(y)$ from (2.14); we get toral groups only. The tables in [CoGr] do not list elements of order 9, but one can classify automorphisms of order 3 in $3 E_{6}(\mathbb{K})$ by, say, $(8.6)$ of [Kac]; the fixed point subgroups have types $A_{2}^{3}, T_{2} D_{4}, T_{1} A_{1} A_{4}, T_{1} D_{5}$ and $T_{1} A_{5}$; in 
the first case, $y$ would have order 3 and in all other cases, we get toral groups only.

Getting the structures of the normalizers is an exercise. Recall that $G$ has no Chevalley involutions (2.23); elements in $N(\mathbb{T})$ which represent -1 in the Weyl group square to a generator of $Z(G)$, which lies in the $\mathbb{T}_{1}$-factor; use (9.5) to prove nonsplitting.

\section{EXTRASPECIAL SUBGROUPS OF $E_{8}(\mathbb{K})$}

As an application of our results, we study extraspecial subgroups of $G:=E_{8}(\mathbb{K})$ and show how to locate the maximal ones; see (1.8), Table IV. Except for certain extraspecial 2-groups with a 2B-involution in the center, it is easy to prove that the groups are small and analyze with earlier results, so we treat only the case $p=2$.

(12.1) NOTATION. Let $P$ be an extraspecial 2-group of order $2^{1+2 d}$ in $E_{8}(\mathbb{K})$ with $z$ a generator for $Z(P)$.

(12.2) LEMMA. Suppose $z \in 2$ B. There are, up to conjugacy, two maximal extraspecial 2-groups in $C(z) \cong H \operatorname{Spin}(16, \mathbb{K})$, they are isomorphic to $2_{+}^{1+2 r}$ for $r=3$ and 7 .

Proof. Let $\pi: H \rightarrow C(z)$, where $H=\operatorname{Spin}(16, \mathbb{K})$. Let $P$ be a maximal extraspecial subgroup of $C(z), P=\langle z\rangle$ and $Q:=P \pi^{-1}$; then $Q / Z(H)$ is elementary abelian. Let $Z$ be the kernel of $H$ acting on the natural module $V$ of dimension 16.

Suppose $Q / Z$ is elementary abelian. Then $Q / Z$ is in a diagonal frame group and $Q$ has the form $2_{+}^{1+14} \times 2$. Since $Q^{\prime}=Z$, we get an extraspecial subgroup $Q \pi=2_{+}^{1+14}$, unique up to conjugacy.

Suppose $Q / Z$ is not elementary abelian. From (2.22.ii), if only one spin type is present, $\Phi(Q / Z)=\Phi(Q) Z / Z, \Phi(Q) Z=\Phi(Q) \times Z$ and $Q / Z \cong P$; maximality gives $P \cong 2_{+}^{1+6}$. We claim that only one spin type occurs (if so we are done). Suppose both spin types are present. Then, in the notation of (2.22.ii), $\left[Q^{+}, Q^{-}\right]=1$ and, for some $\varepsilon,\left\langle Q^{\varepsilon}\right\rangle \geqslant \operatorname{ker}(\pi)$, whence $\left\langle Q^{\varepsilon}\right\rangle^{\pi}$ is a nontrivial central direct factor of the extraspecial group $P$, a contradiction.

(12.3) PROPOSITION. For any $z \in \mathbf{2 A}$, let $P \cong 2_{\varepsilon}^{1+2 d}$ be a maximal extraspecial group, $\langle z\rangle=Z(P)$. Then $d \leqslant 2$. Also, $P$ is a subgroup of a group of the form $A \times R$, as follows. There are quaternion groups $P_{1}$ and $P_{2}$, where the $P_{i}$ lie in the distinct factors of $C(x) \cong 2 E_{7}(\mathbb{K}) A_{1}(\mathbb{K})$ (there are three possibilities for $P_{1}$ and one for $P_{2}$, up to conjugacy), $A \cong 4 \times 2$ or $2^{3}$ and $\left|A \cap P_{1} P_{2}\right|=2$.

Proof. Use (9.5) and (12.2). 


\section{ConCluding REMARKS}

(13.1) Results in the spirit of complexity are likely to exist for $p=2$ in the nonsimply connected cases, though we have not worked them out. Here is an easy result of this kind for type $A$. Let $G=\operatorname{SL}(n, \mathbb{R})$, with $n$ even, and let $Z$ be an even order subgroup of $Z:=Z(G)$. Base the notion of complexity in $G / Z$ on the class of $Z x$, where $x$ is conjugate to $\operatorname{diag}\left(i^{n / 2},-i^{n / 2}\right)$. If $Z \leqslant E \leqslant G$ and $E / Z$ is elementary abelian, $E$ is a central product $Q \circ\left(A Z_{1}\right)$, where $Q$ is extraspecial, $A$ is elementary abelian, $Z_{1}$ is cyclic and contains $Z$ and $\left[A, Z_{1}\right] \leqslant \Omega_{1}(Z)$. Noncentral elements of $Q A$ which square to -1 are precisely the elements of the class of $x$. Now make the special assumption that $Z=Z_{1}$. The group $E$ is toral iff $Q=1$ iff the complexity of $E / Z$ is at most 1 .

(13.2) For $p$ odd, complexity results are elusive. Inspection of the $E_{8}(\mathbb{K})$ case for $p=3$ reveals examples of pairs of groups $\left(E_{r}, F_{r}\right)$, where $\operatorname{rk}\left(E_{r}\right)=\operatorname{rk}\left(F_{r}\right)=r \in\{3,4\}$, there exist isomorphisms $\varphi_{r}: E_{r} \rightarrow F_{r}$ such that $x$ and $x \varphi_{r}$ are in the same $E_{8}(\mathbb{K})$-class, for all $x \in E_{r}$, but $E_{r}$ is toral and $F_{r}$ is not. For instance, we may take $E_{r}$ in the radical of a type 2 maximal, $F_{4}$ the radical of a type 1 maximal and $F_{3}$ a complement to the socle in $F_{4}$. These fourgroups are 3B-pure. Furthermore, their normalizers induce on them the groups

$$
\begin{array}{lll} 
& E_{r} & F_{r} \\
r=3: & 3^{2}: \operatorname{GL}(2,3) \times 2 & \operatorname{GL}(3,3) \\
r=4: & \operatorname{Sp}(4,3) \cdot 2 & 3^{3}: \operatorname{GL}(3,3) .
\end{array}
$$

It follows that, for $r \in\{3,4\}$, there exist monomorphisms of an elementary abelian group of order $3^{r}$ to $E_{8}(\mathbb{K})$ such that the two images of every element are conjugate, yet the monomorphisms do not differ by conjugation with an element of $E_{8}(\mathbb{K})$. Serre called my attention to this question: for a particular Lie group $G$ and two embeddings $\varphi_{i}$ of a finite group $F$ into $G$ such that $x \varphi_{1}$ and $x \varphi_{2}$ are conjugate for all $x \in F$, is there $g \in G$ such that $x \varphi_{2}=g^{-1}\left(x \varphi_{1}\right) g$, for all $x \in G$ ? This is true, for example, for $\operatorname{SL}(n, \mathbb{C})$ but false for $\operatorname{PSL}(n, \mathbb{C})$ (easy) and $E_{8}(\mathbb{C})$, as the above remarks show.

On the positive side of the ledger, one has Theorem $B$ of [Bo], which says in part that if $G$ is a compact and connected Lie group, then every elementary abelian $p$-group is contained in a torus iff that is so for elementary abelian groups of rank at most 3; the proof depends on the classification of simple algebraic groups. Of course, this statement may be deduced from the results of this paper.

In [Bo], one finds that if $G$ is nearly simple, connected and simply connected and $|Z(G)|$ is divisible by the prime $p$, then for an element $z \in Z(G)$ 
of order $p$, there is an extraspecial group $P$ of order $p^{3}$ in $G$ with $\langle z\rangle=Z(P)$ and $P$ quaternion if $p=2$ and $\exp (P)=p$ if $p$ is odd; this, too, follows from this article. An analogous statement for central extensions of finite simple group would be false, e.g. $2 \cdot M_{22}$.

(13.3) The result for $E_{8}(\mathbb{K})$, (8.4), came before most of the others. The statement is neat, at least if one can forgive the eccentric rank 5 2B-pure example. Our analysis of the orthogonal groups and examples of nontoral elementary abelian 2-subgroups, suggests that such eccentric groups are really a generic part of the theory. The reason is that the set of conjugacy classes used to define complexity could be avoided by nontoral groups $E$ corresponding to codes of high minimum weight (see (5.3)); in this case the characteristic function would be identically 0 on $E$.

Alternatively, if a nontoral $E$ is allowed to meet classes defining complexity, the complexity can become quite high. In the example (5.3), $E^{\#}$ consists of conjugate involutions, so the complexity of $E$ would be $k$, its dimension, yet $E$ is nontoral.

It would be interesting if complexity were replaced by a notion which doesn't make certain nontoral groups an exception to an otherwise simply stated criterion.

(13.4) One would like 'natural' explanations of the rather exotic looking normalizers in Tables I and II.

The nonsplit extension $2^{3}$. GL $(3,2)$ occurs in $G_{2}(\mathbb{K})$ and as the automorphism group of a Moufang loop, $\mathbb{O}_{16}$; see [Gr3] for several uses of this loop for constructions of so-called parabolic subgroups of sporadic simple groups. In a different direction, we used $\mathbb{O}_{16}$ to construct the subgroup $2^{2+3+2 \cdot 3} \cdot\left[\mathrm{GL}(3,2) \times \Sigma_{3}\right]$ of $\operatorname{Spin}(8, \mathbb{K}): \Sigma_{3}$. This group occurs as the normalizer of the unique maximal elementary abelian 2-group in $F_{4}(\mathbb{K})$; this follows directly from the containment of $\operatorname{Spin}(8, \mathbb{K}): \Sigma_{3}$ in $F_{4}(\mathbb{K})$.

Recently, some progress has been made for the prime 3 [Gr4]. The subgroup $3^{3}: \mathrm{SL}(3,3)$ of $F_{4}(\mathbb{K})$ and its associated decomposition of the 27dimensional exceptional Jordan algebra may be explained by a Moufang loop of order 81 . The construction of an associated cubic form explains the group $3^{1+3+3}: \operatorname{SL}(3,3)$ in $3 E_{6}(\mathbb{K})$. It is an analogue for $p=3$ of the phenomena mentioned in the previous paragraph.

\section{APPENDIX 1: A PROOF OF ADAMS' THEOREM}

J. Frank Adams circulated a preprint in 1986 which classified the maximal elementary abelian subgroups of $E_{8}(\mathbb{C})$. We give here an alternate proof of his theorem (2.17). In the arguments below, we use results of this paper proved 
before Section 9, where we quoted Adams' theorem. We use an algebraically closed field $\mathbb{K}$ of characteristic not 2 .

That two maximal elementary abelian groups exist in $G$ is easy to prove; existence is sketched in the statement of (2.17) and the verification of the stated properties of their normalizers is a routine calculation in $G_{2}(\mathbb{K}) \times F_{4}(\mathbb{K})$ and $2^{2} D_{4}(\mathbb{K})^{2}:\left[2 \times \Sigma_{3}\right]$.

(A1.1) NOTATION. $G=E_{8}(\mathbb{K})$ and $E$ is a maximal elementary abelian group. We refer to (2.14) for information on conjugacy classes.

(A1.2) LEMMA. Suppose E contains a fours-group $U$ with distribution $A^{3}$. Then, $E$ is conjugate to one of the two groups in (2.17).

Proof. From [CoGr, (3.8)], $C(U)^{\circ} \cong \mathbb{T}_{2} \circ 3 E_{6}(\mathbb{K})$ and $C(U)=C(U)^{\circ}\langle t\rangle$, where $t$ has order 2 and induces a graph automorphism on $X$, the $3 E_{6}(\mathbb{K})$ component and inverts $T$, the $\mathbb{T}_{2}$-component. We quote (2.18), row $E_{6}$, then (2.21), and (7.3) to get at most four possibilities for $E$.

We claim that the two rank 8 cases are conjugate by $X$. Consider $U$, the rank 5 group coming from fixed points of type $C_{4}$. Use $(2.21)(1,4,0,4)$ then (2.26) and (2.16.iii) to get a 2A-pure eights-group in $U$. By (8.2), $U$ is nontoral and the claim follows. These groups are type 2 .

The rank 9 cases here lead to type 1 since $(E \cap T)(E \cap X)$ is toral, by (8.2).

What remains is for us to verify the hypothesis of (A1.2). We deny it and seek a contradiction. We may assume that $E$ is nontoral, so that by (2.13.vi), $\mathrm{rk}(E) \geqslant 3$.

(A1.3) HYPOTHESIS. E contains no fours-group with distribution $A^{3}$.

(A1.4) LEMMA. $E$ does not contain a fours-group with distribution $A B^{2}$.

Proof. Suppose $U$ is such a subgroup. Then [CoGr, (3.8)] gives $C(U) \cong 4 A_{7} \mathbb{T}_{1}: 2$ (actually, it gives only the factor types and component group, $\mathbb{Z}_{2}$; we use the fact that $E_{8}(\mathbb{K})$ has a Chevalley involution, (2.23); note also that the two factors of $C(U)^{0}$ intersect in a group of order 2 (containing the $2 \mathrm{~A}$ involution of $U$ ) since $C(U)$ lies in a $2 E_{7}(\mathbb{K}) A_{1}(\mathbb{K})$ subgroup.

Let $H$ be the $4 A_{7}$-factor, so that $H \cong \operatorname{SL}(8, \mathbb{K}) /\{ \pm 1\}$. A maximal torus $S$ of $H$ lies in $\mathbb{T}$, a maximal torus of $G$ and $\left|\mathbb{T}_{(2)}: S \cap \mathbb{T}_{(2)}\right|=2$. It follows from (2.16.ii) that $S$ contains a fours-group with distribution $A^{3}$.

Define $F:=E \cap C(U)^{0}$. If $E=F$, and $E$ is toral, we may assume $E$ lies in $S$. Maximality implies $E=S$ and so we contradict (A1.2). If $E=F$ is not toral, maximality of $E,(3.1)$ and (2.26) contradict (A1.2).

We may suppose now that $F<E$. Let $e \in E \backslash F$. From (2.18), row $A$, we get that $F$ lies in $C_{C(U)^{\circ}}(e)=K \times 2 \times 2$, where $K \cong \mathrm{PSO}(8, \mathbb{K})$ or $\operatorname{PSp}(8, \mathbb{K})$.

In case $K \cong \operatorname{PSp}(8, \mathbb{K})$, if $\operatorname{rk}(F)=6, F$ is toral in $G(1.8)$, Table III, row $E_{6}$. 
Considering a $3 A_{2} E_{6}$-subgroup of $G$ which contains our $K$, we see that $F$ is a nonsingular rank 6 subspace of nonmaximal Witt index in $\mathbb{T}_{(2)}$, in conflict with (A1.3). If $F$ is a rank 5 maximal of $K$, it is a rank 5 maximal of a natural $F_{4}$-subgroup of $G$ and so contains a $2 \mathrm{~A}$-pure eights-group, again contradicting (A1.3).

In case $K \cong \operatorname{PSO}(8, \mathbb{K})$, we use (5.4) and (5.5) to get that $F$ is the image of a diagonal frame group, the image of a toral group of exponent 4 or a nonabelian group as in (2.19). Using (2.14) and (2.26), we contradict (A1.3).

(A1.5) LEMMA. E $\cap 2 \mathrm{~A}$ is nonempty.

Proof. Otherwise, $E$ is 2B-pure and [CoGr, (3.8)] tells us that $\operatorname{rk}(E) \leqslant 5$ and gives the structure of $C(E)$. However, $E$ then is not maximal abelian, for $C(E)$ contains a conjugate of $\mathbb{T}_{(2)}$.

(A1.6) LEMMA. E $\cap$ 2B generates a subgroup, $F$, of index 2 in $E$ and $F^{\#}=E \cap \mathbf{2 B}$. Consequently, $E \backslash F=E \cap \mathbf{2 A}$.

Proof. Use (A1.5), then (A1.3) and (A1.4).

(A1.7) LEMMA. Let $r=\operatorname{rk}(F)$. Then $r \leqslant 3$.

Proof. Suppose $r \geqslant 4$. Let $D$ be a subgroup of $E$ generated by five independent elements of $2 \mathrm{~A} \cap E$. Then $\operatorname{rk}(D)=5$ and, letting tr denote trace on the adjoint module, we have $\Sigma_{g \in D} \operatorname{tr}(g)=248+16 \cdot 24+15(-8)=512$, whence $\operatorname{dim} C(D)=512 / 32=16$. From [CoGr, (3.8)], we have that $C(F \cap D) \cong \pi \cdot 2_{+}^{1+6}$ has dimension only 8 , a contradiction.

(A1.8) THE FINAL CONTRADICTION. We have $\operatorname{rk}(E)=3$ or 4 . It is almost trivial to prove that $E$ is not a maximal elementary abelian group.

If $\operatorname{rk}(E)=3, C(F) \cong 2^{2} D_{4}(\mathbb{K})^{2}: 2$ (again, by [CoGr, (3.8)]). Let $x \in E \backslash F$. If $x \notin C(F)^{0}, x$ interchanges the two factors and so has fixed point subgroup $\operatorname{PSO}(8, \mathbb{K})$, which contains involutions, in violation of maximality of $E$. Consequently $x \in C(F)^{\circ}$ and so $x$ and therefore $E$ are in a torus $\mathbb{T}$ of $C(F)$ and so are in a group $\mathbb{T}_{(2)}$, again in conflict with maximality.

If $\operatorname{rk}(E)=4, \operatorname{rk}(F)=3$ and so $[\mathrm{CoGr},(3.8)]$ tells us that $C(F)^{0} \cong 2^{4} A_{1}(\mathbb{K})^{8}$ and $E=Z\left(C(F)^{\circ}\right)$. Since $C(F)^{0}$ has a rank 8 torus, there certainly are involutions in $C(E) \backslash E$. This is our final contradiction.

In the second case, $\operatorname{rk}(F)=7$ and $F$ is not toral in $K$ but is toral in $H$ and so is a hyperplane of some $\mathbb{T}_{(2)}$ in $G$. This contradicts the hypothesis of (A1.4).

\section{APPENDIX 2: LifTING}

We describe a sufficient condition for lifting a subgroup of a Chevalley group over one ring to a Chevalley group over another ring. 
(A.1) NOTATION. Let $G(\cdot)$ be a functor from commutative rings to groups which gives a simply connected Chevalley group when applied to $\mathbb{K}$ and the universal Steinberg group when applied to finite fields and suppose also that it commutes with inverse limits (see, e.g., the procedure of Section A in [Bo2]).

For an ideal $J$ of the ring $R, G(R, J)$ denotes the kernel of the map $G(R) \rightarrow G(R / J)$.

(A2.2) NOTATION. Given the finite field $F$ of $q$ elements, with $q$ a power of the prime $p$, we let $R$ be the ring $\mathbb{Z}[\zeta]$, where $\zeta=\exp (2 \pi i /(q-1)), \mathscr{P}$ a prime ideal containing $p$. Let $\hat{R}$ be the $\mathscr{P}$-adic completion.

For an integer $k \geqslant 1$, define $R_{k}:=R / \mathscr{P}^{k}$. We let $\eta_{i j}$ be the natural epimorphism $R_{i} \rightarrow R_{j}$, defined iff $i \geqslant j$. The functor $G(\cdot)$ then gives corresponding maps $\varphi_{i j}$ of groups $G_{i}:=G\left(R_{i}\right)$. We take the index set $I$ to be all nonnegative integers.

(A2.3) LEMMA. Let $K$ be the fraction field of $\hat{R}$. Then, $K$ has cardinality $2^{\aleph_{0}}$ and there is an embedding of $K$ into $\mathbb{C}$.

Proof. Let $S=\{0,1, \ldots, p-1\}$. The set of $p$-adic integers is, via taking a power-series representation with coefficients in $S$, set-isomorphic to the set of all formal sequences $S^{\mathbb{N}}$, whence the set of $p$-adic integers has cardinality $2^{\aleph_{0}}$. The same holds for $R$, a finite extension of the $p$-adics, and for $K$. In general, if $E$ is an infinite field and $X$ a set of variables, $E$ and its algebraic closure have the same cardinalities and the cardinality of $E[X]$ is the larger of card $(E)$ and card $(X)$. It follows that the transcendence degree of $K$ over $\mathbb{Q}$ is $2^{\mathrm{N}_{0}}$. At once, there is an embedding of $K$ into $\mathbb{C}$.

(A2.4) LIFTING CRITERION. Let $p$ be a prime number and $F$ a field of characteristic p. Let $R$ be a complete discrete valuation ring with $R / J \cong F$, where $J$ is the radical of $R$. Assume also that $\operatorname{card}(R) \leqslant 2^{N_{0}}$. Suppose $G(\cdot)$ is a Chevalley group functor and $H$ is a subgroup of $G(F)$ such that $H^{2}(H, M)=0$, where $M \cong G(R, J) / G\left(R, J^{2}\right)$. Then, there is a subgroup of $G(R)$ whose image is $H$ under the natural map $G(R) \rightarrow G(F)$.

Proof. For any $n \geqslant 0, G\left(R, J^{n}\right) / G\left(R, J^{n+1}\right) \cong M$. To see this, notice that we get a morphism of modules $G\left(R, J^{n}\right) / G\left(R, J^{n+1}\right) \rightarrow G\left(R, J^{n+1}\right) / G\left(R, J^{n+2}\right)$ from the $p$ th power map.

Let $q_{n}: R / J^{n+1} \rightarrow R / J^{n}$ be the quotient of rings and $p_{n}:=G\left(q_{n}\right)$ the associated quotient of groups. Since $H^{2}(H, M)=0$, there is, by induction, a homomorphism $\psi_{n}: H \rightarrow G\left(R / J^{n}\right)$ such that $\psi_{n} p_{n-1}=\psi_{n-1}$. We get a homorphism $\psi: H \rightarrow G(R)$ by 'taking the limit', i.e. $\psi(h):=\lim _{n} \psi_{n}(h)$ for $h \in H$, and its image has the required property. Notice that we have used the commuting of inverse limits and $G(\cdot)$ here. 
(A2.5) REMÁRK. Actually, the module $M$ for $G(F)$ in (A2.4) is the adjoint module for the group $G(F)$ (i.e. the tensor of $F$ with the $\mathbb{Z}$-form spanned by the Chevalley basis), but we do not need this fact. Observe that if $G(\cdot)$ is based on the root system $\Sigma$ and $\Gamma$ is a parabolic subsystem, then we have a corresponding subfunctor $G_{0}(\cdot)$ of $G(\cdot)$ and the corresponding module $M_{0}$ is a submodule of $M$, as a $G_{0}(F)$-module. In case $\Gamma$ is a root system, 'submodule' may be replaced by 'direct summand' in the previous statement about modules. Thus, if $G_{0}(\cdot)$ gives the Levi factor of a parabolic subgroup $P(\cdot)$ of $G(\cdot), M_{0}$ occurs naturally as a $P(F)$-subquotient module of $M$.

(A2.6) REMARK. If $H$ is a finite subgroup of $G(F)$ and $p=\operatorname{char}(F)$ divides $|H|$, we get a nonsplit extension of $H$ over $G(R, J)$ in some cases. A sufficient condition is that the degree of the minimal polynomial of the adjoint representation of every element of order $p$ in $G(F)$ be less than $p$; this requires $p \geqslant 5$ (see [Gr7 (5.III)]; unfortunately the result is misstated there). To prove this, we may assume that $H=\langle x\rangle$ has order $p$ and that the root system for $G$ is indecomposable. Let $H \leqslant U$, a maximal unipotent group of $G(F)$. If there is a parabolic $P \geqslant U$ such that $x \notin O_{p}(P)$, we use induction (in the notation of (A2.5), there is a submodule with quotient $M_{0}$ in which $y^{p} \neq 1$, where $y G(R, J)=x$; the hypothesis on the canonical form insures that no element of $y G(R, J)$ has $p$ th power in $\left.G\left(R, J^{2}\right)\right)$. If there is no such $P$, when we write $x$ as a product of root elements; each root which occurs has height at least 2 . There is an element $n \in G(F)$ corresponding to a simple reflection such that the least root in the support of $x^{n} \in U$ is less than that of $x$. Induction on the height of this least root then shows that if $y G(R, J)=x, y^{p} \notin G\left(R, J^{2}\right)$.

(A2.7) COROLLARY. Suppose that char $F=p>0$ and that $H$ is a finite subgroup of $G(F)$ of order prime to $p$. Then $H$ lifts to a subgroup of $G(K)$, where $K$ is a subfield of $\mathbb{C}$.

\section{REFERENCES}

[Ad] Adams, J. F., 'Elementary abelian 2-groups in $E_{8}(\mathbb{C})$ ', preprint (1986).

[Alek] Alekseevskii, A. V., 'Finite commutative Jordan subgroups of complex simple Lie groups', Functional Anal. Appl. 8 (1974), 277-279.

[Bo] Borel, A., 'Sous groupes commutatifs et torsion des groupes de Lie compacts connexes', Tohoku Math. J. (2) 13 (1961), 216-240.

[Bo2] Borel, A., et al., 'Seminar on algebraic groups and related finite groups', Lecture Notes in Math. 131, Springer Verlag, Berlin, 1970.

[BoSe] Borel, A. and Serre, J. P., 'Sur certains sous groupes des groupes de Lie compacts', Comment. Math. Helv. 27 (1953), 128-139.

[BoMo] Borel, A. and Mostow, G. D., 'On semisimple automorphisms of Lie algebras', Ann. Math. (2) 61 (1955), 389-405. 
[Chev] Chevalley, C., The Algebraic Theory of Spinors, Columbia Univ. Press, Morningside Heights, 1954.

[CoGr] Cohen, A. M. and Griess, R., Jr, 'On finite simple subgroups of the complex Lie group of type $E_{8}{ }^{\prime}$, Proc. Symp. Pure Math. 47 (1987), 367-405.

[CoLiSaSe] Cohen, A. M., Liebeck, M., Saxl, J. and Seitz, G., 'The local maximal subgroups of the exceptional groups of Lie type', preprint.

[CoWa] Cohen, A. M. and Wales, D., 'Finite subgroups of $F_{4}(\mathbb{C})$ and $E_{6}(\mathbb{C})$ ', preprint.

[Cu ] Curran, P., 'Fixed point free action on a class of abelian groups', Proc. Amer. Math. Soc. 57 (1976), 189-193.

[de Sieb] de Siebenthal, J., 'Sur les groupes de Lie compacts non connexes', Comment. Math. Helv. 31 (1956), 41-89.

[GagGar] Gagola, S. and Garrison, S., 'Real characters, double covers and the multiplier', $J$. Algebra.

[Gant] Gantmacher, F., Rec. Math. Moscou N.S. 5 (1939), 101-144.

[Go] Gorenstein, D., Finite Groupes, Harper \& Row, New York, 1968.

[GoLy] Gorenstein, D. and Lyons, R., 'The local structure of finite groups of characteristic two type', Mem. Amer. Math. Soc. 42 (1983).

[Gr1] Griess, R. L. Jr, 'Splitting of extensions of $S L(3,3)$ by the vector space $\mathbb{F}_{3}^{3}$, Pacific $J$. Math. 63 (1976), 405-409.

[Gr2] Griess, R. L. Jr, 'Quotients of infinite reflection groupes', Math. Annal. 263 (1983), 268-278.

[Gr3] Griess, R. L. Jr, 'Sporadic groups, code loops and nonvanishing cohomology', $J$. Pure Appl. Algebra 44 (1987), 191-214.

[Gr4] Griess, R. L. Jr, "A Moufang loop, the exceptional Jordan algebra and a cubic form in 27 variables' (submitted to J. Algebra).

[Gr5] Griess, R. L. Jr, 'Code loops and a large finite group containing triality for $D_{4}$ ', from Atti Conv. Inter. Teoria Geom. Combin. Firenze, 23-22 Ottobre, 1986; Supplemento as Rend. Circ. Mat. Palermo, 79-98.

[Gr6] Griess, R. L. Jr, 'The Friendly Giant', Invent. Math. 69 (1982), 1-102.

[Gru] Gruenberg, K., Cohomological Topics in Group Theory, Springer, Berlin, 1970.

[Hum] Humphreys, J., Introduction to Lie Algebras and Representation Theory, Springer, New York, 1972.

[Hup] Huppert, B., Endliche Gruppen I, Springer-Verlag, Berlin, 1968.

[Kac] Kac, V. G., Infinite Dimensional Lie Algebras (2nd edn), Cambridge Univ. Press, Cambridge, 1985.

[K1] Kleidman, P., Meierfrankenfeld, U. and Ryba, A., 'The Rudvalis group is in $E_{7}(7)$,, in preparation.

[Ly] Lyons, R., 'Evidence for a new finite simple group', J. Algebra 20 (1972), 540-569.

[McL] McLaughlin, J. E., personal communication, 1975.

[MacW-Sl] MacWilliams, F. J. and Sloane, N. J. A., The Theory of Error Correcting Codes, North Holland, Amsterdam, 1977.

[MoPa] Moody R. and Patera, J., 'Characters of elements of finite order in Lie groups', SIAM J. Algebra Discrete Methods 5 (1984), 359-383.

[Ro] Robinson, D., 'The vanishing of certain homology and cohomology groups', $J$. Pure Appl. Algebra 7 (1976), 145-167.

[Sah] Sah, C.-H., 'Cohomology of split group extensions', J. Algebra 29 (1974), 255-302.

[Sch1] Schur, I., 'Über die Darstellung der endlichen Gruppen durch gebrochene lineare Substitutionen', J. Reine Angew. Math. 127 (1904), 20-50.

[Sch2] Schur, I., 'Über die Darstellung der endlichen Gruppen durch gebrochene lineare Substitutionen', J. Reine Angew. Math. 132 (1907), 85-107.

[Sch3] Schur, I., 'Über die Darstellung der symmetrischen und alternienden Gruppen durch gebrochene lineare Substitutionen', J. Reine Angew. Math. 139 (1911), 155250 . 
[SS] Springer, T. and Steinberg, R., 'Conjugacy Classes', Section E of [Bo2].

[St] Steinberg, R., 'Generators, relations and coverings of algebraic groups', J. Algebra II 71 (1981), 521-543.

[Wood] Wood, J. A., 'Spinor groups and algebraic coding theory', J. Combin. Theory (1989), 277-313.

[Yo] Young, K. C., 'Some simple subgroups of the Tits simple group, 72T-B263', Notices Amer. Math. Soc. (1972).

Author's address:

Robert L. Griess, Jr,

Department of Mathematics,

University of Michigan,

Ann Arbor, MI 48109,

U.S.A.

(Received, January 8, 1990; revised version, May 1, 1991) 